\title{
Thermal conductivity estimation of diverse liquid aliphatic oxygen-containing organic compounds using QSPR method
}

Haixia Lu, Wanqiang Liu*, Fan Yang, Hu Zhou, Fengping Liu, Hua Yuan, Guanfan Chen, and Yinchun Jiao*

School of Chemistry and Chemical Engineering, Key Laboratory of Theoretical Organic Chemistry and Function Molecule of Ministry of Education,

Hunan Province College Key Laboratory of QSAR/QSPR, Hunan Provincial Key Laboratory of Controllable Preparation and Functional Application

of Fine Polymers, Hunan University of Science and Technology, Xiangtan, 411201, China

E-mail: wanqiangliu@hnust.edu.cn;

yinchunjiao@hnust.edu.cn 


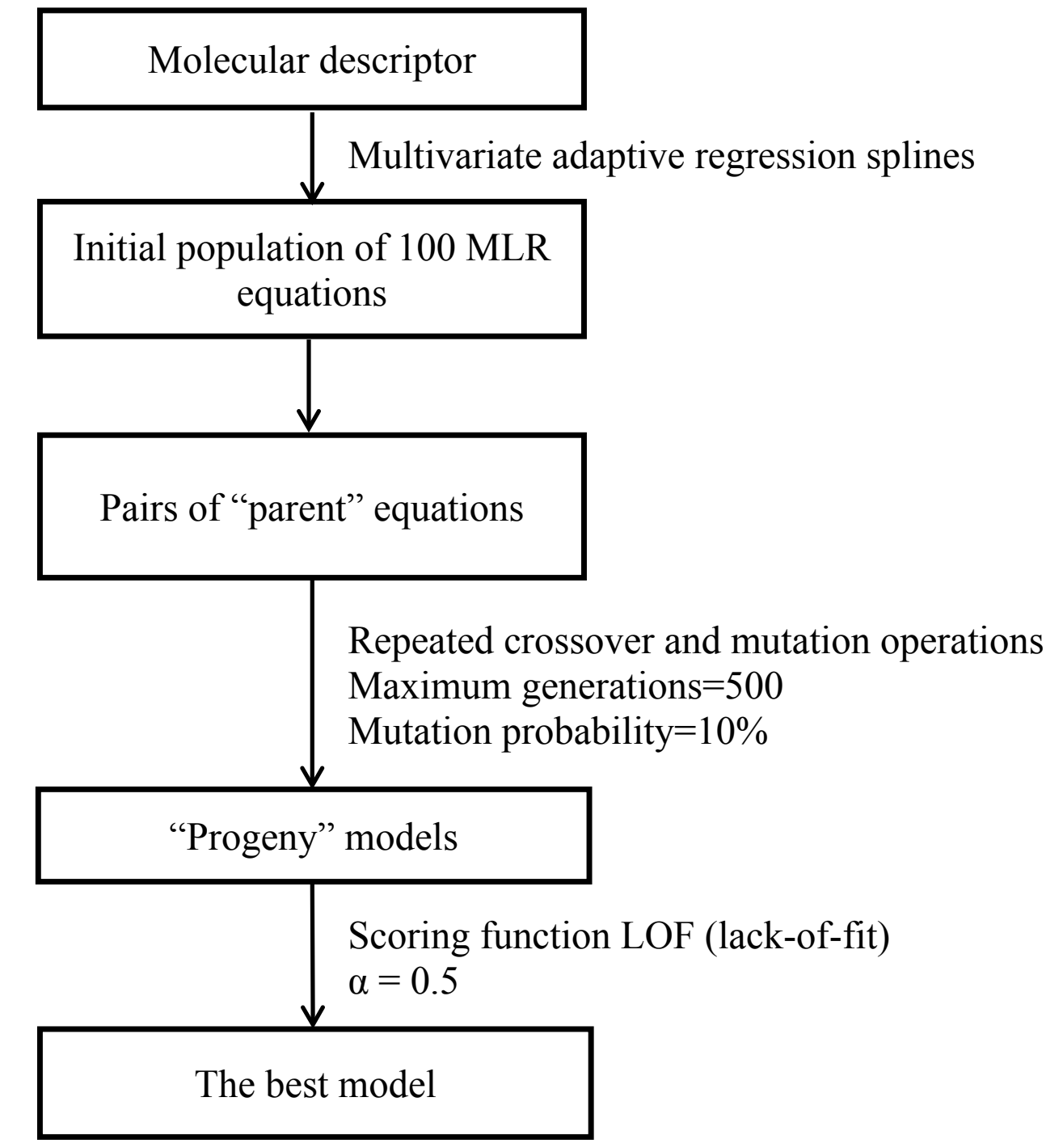


Figure S1. Flowchart of GFA process

Table S1. Chemicals involved in this work

\begin{tabular}{|c|c|c|c|c|c|c|}
\hline NO. & IUPAC name & CAS number & Structure & Reference & Purity (mass fraction) & Source \\
\hline 1 & Methanol & $67-56-1$ & $\mathrm{CH}_{3} \mathrm{OH}$ & 1 & NA & $\mathrm{NA}$ \\
\hline 2 & Ethanol & $64-17-5$ & $\mathrm{CH}_{3} \mathrm{CH}_{2} \mathrm{OH}$ & 1 & NA & NA \\
\hline 3 & n-Propyl alcohol & $71-23-8$ & $\mathrm{CH}_{3}\left(\mathrm{CH}_{2}\right)_{2} \mathrm{OH}$ & 1 & NA & $\mathrm{NA}$ \\
\hline 4 & Isopropyl alcohol & $67-63-0$ & $\left(\mathrm{CH}_{3}\right)_{2} \mathrm{CHOH}$ & 1 & NA & NA \\
\hline 5 & n-butanol & $71-36-3$ & $\mathrm{CH}_{3}\left(\mathrm{CH}_{2}\right)_{3} \mathrm{OH}$ & 1 & NA & NA \\
\hline 6 & isobutyl alcohol & $78-83-1$ & $\left(\mathrm{CH}_{3}\right)_{2} \mathrm{CHCH}_{2} \mathrm{OH}$ & 1 & NA & $\mathrm{NA}$ \\
\hline 7 & tertbutyl alcohol & $75-65-0$ & $\left(\mathrm{CH}_{3}\right)_{3} \mathrm{COH}$ & 1 & NA & NA \\
\hline 8 & n-amyl alcohol & $71-41-0$ & $\mathrm{CH}_{3}\left(\mathrm{CH}_{2}\right)_{4} \mathrm{OH}$ & 1 & NA & NA \\
\hline 9 & isoamyl alcohol & $123-51-3$ & $\left(\mathrm{CH}_{3}\right)_{2} \mathrm{CH}\left(\mathrm{CH}_{2}\right)_{2} \mathrm{OH}$ & 1 & NA & $\mathrm{NA}$ \\
\hline 10 & 1-hexanol & $111-27-3$ & $\mathrm{CH}_{3}\left(\mathrm{CH}_{2}\right)_{5} \mathrm{OH}$ & 1 & NA & $\mathrm{NA}$ \\
\hline 11 & 2-hexanol & $626-93-7$ & $\mathrm{CH}_{3}\left(\mathrm{CH}_{2}\right)_{3} \mathrm{CH}\left(\mathrm{CH}_{3}\right)_{2} \mathrm{OH}$ & 1 & NA & $\mathrm{NA}$ \\
\hline 12 & 1-heptanol & $111-70-6$ & $\mathrm{CH}_{3}\left(\mathrm{CH}_{2}\right)_{6} \mathrm{OH}$ & 1 & NA & NA \\
\hline 13 & isoheptanol & $51774-11-9$ & $\left(\mathrm{CH}_{3}\right)_{2} \mathrm{CH}\left(\mathrm{CH}_{2}\right)_{4} \mathrm{OH}$ & 1 & NA & NA \\
\hline 14 & tert-heptanol & $625-23-0$ & $\mathrm{CH}_{3}\left(\mathrm{CH}_{2}\right)_{3} \mathrm{C}\left(\mathrm{CH}_{3}\right)_{2} \mathrm{OH}$ & 1 & NA & $\mathrm{NA}$ \\
\hline 15 & n-decyl alcohol & $112-30-1$ & $\mathrm{CH}_{3}\left(\mathrm{CH}_{2}\right)_{9} \mathrm{OH}$ & 1 & NA & NA \\
\hline 16 & n-undecyl alcohol & $112-42-5$ & $\mathrm{CH}_{3}\left(\mathrm{CH}_{2}\right)_{10} \mathrm{OH}$ & 1 & NA & $\mathrm{NA}$ \\
\hline 17 & n-dodecyl alcohol & $112-53-8$ & $\mathrm{CH}_{3}\left(\mathrm{CH}_{2}\right)_{12} \mathrm{OH}$ & 1 & NA & NA \\
\hline 18 & 1-tetradecanol & $112-72-1$ & $\mathrm{CH}_{3}\left(\mathrm{CH}_{2}\right)_{13} \mathrm{OH}$ & 1 & NA & NA \\
\hline 19 & n-octadecyl alcohol & $112-92-5$ & $\mathrm{CH}_{3}\left(\mathrm{CH}_{2}\right)_{17} \mathrm{OH}$ & 1 & NA & NA \\
\hline 20 & n-octyl alcohol & $111-87-5$ & $\mathrm{CH}_{3}\left(\mathrm{CH}_{2}\right)_{7} \mathrm{OH}$ & 1 & NA & NA \\
\hline 21 & sec-octyl alcohol & $123-96-6$ & $\mathrm{CH}_{3}\left(\mathrm{CH}_{2}\right)_{5} \mathrm{CH}\left(\mathrm{CH}_{3}\right) \mathrm{OH}$ & 1 & NA & NA \\
\hline 22 & n-nonyl alcohol & $143-08-8$ & $\mathrm{CH}_{3}\left(\mathrm{CH}_{2}\right)_{8} \mathrm{OH}$ & 1 & NA & NA \\
\hline
\end{tabular}




\begin{tabular}{|c|c|c|c|c|c|c|}
\hline 23 & diethyl ether & $60-29-7$ & $\mathrm{CH}_{3} \mathrm{CH}_{2} \mathrm{OCH}_{2} \mathrm{CH}_{3}$ & 1 & NA & $\mathrm{NA}$ \\
\hline 24 & diisopropyl ether & $108-20-3$ & $\left(\mathrm{CH}_{3}\right)_{2} \mathrm{CHOCH}\left(\mathrm{CH}_{3}\right)_{2}$ & 1 & NA & NA \\
\hline 25 & dipropyl ether & $111-43-3$ & $\mathrm{CH}_{3}\left(\mathrm{CH}_{2}\right)_{2} \mathrm{O}\left(\mathrm{CH}_{2}\right)_{2} \mathrm{CH}_{3}$ & 1 & NA & $\mathrm{NA}$ \\
\hline 26 & ethyl-n-butyl ether & $628-81-9$ & $\mathrm{CH}_{3} \mathrm{CH}_{2} \mathrm{O}\left(\mathrm{CH}_{2}\right)_{3} \mathrm{CH}_{3}$ & 1 & NA & $\mathrm{NA}$ \\
\hline 27 & diamyl ether & $693-65-2$ & $\mathrm{CH}_{3}\left(\mathrm{CH}_{2}\right)_{4} \mathrm{O}\left(\mathrm{CH}_{2}\right)_{4} \mathrm{CH}_{3}$ & 1 & NA & NA \\
\hline 28 & ethyl-octyl ether & $929-61-3$ & $\mathrm{CH}_{3} \mathrm{CH}_{2} \mathrm{O}\left(\mathrm{CH}_{2}\right)_{7} \mathrm{CH}_{3}$ & 1 & NA & NA \\
\hline 29 & di-n-hexyl ether & $112-58-3$ & $\mathrm{CH}_{3}\left(\mathrm{CH}_{2}\right)_{5} \mathrm{O}\left(\mathrm{CH}_{2}\right)_{5} \mathrm{CH}_{3}$ & 1 & NA & NA \\
\hline 30 & di-n-heptyl ether & $629-64-1$ & $\mathrm{CH}_{3}\left(\mathrm{CH}_{2}\right)_{6} \mathrm{O}\left(\mathrm{CH}_{2}\right)_{6} \mathrm{CH}_{3}$ & 1 & NA & NA \\
\hline 31 & octyl ether & $629-82-3$ & $\mathrm{CH}_{3}\left(\mathrm{CH}_{2}\right)_{7} \mathrm{O}\left(\mathrm{CH}_{2}\right)_{7} \mathrm{CH}_{3}$ & 1 & NA & NA \\
\hline 32 & dibutyl ether & $142-96-1$ & $\mathrm{CH}_{3}\left(\mathrm{CH}_{2}\right)_{3} \mathrm{O}\left(\mathrm{CH}_{2}\right)_{3} \mathrm{CH}_{3}$ & 1 & NA & NA \\
\hline 33 & Ethyl hexyl ether & $5756-43-4$ & $\mathrm{CH}_{3} \mathrm{CH}_{2} \mathrm{O}\left(\mathrm{CH}_{2}\right)_{5} \mathrm{CH}_{3}$ & 1 & NA & $\mathrm{NA}$ \\
\hline 34 & Isobutyric aldehyde & $78-84-2$ & $\left(\mathrm{CH}_{3}\right)_{2} \mathrm{CHCHO}$ & 1 & NA & NA \\
\hline 35 & valeraldehyde & $110-62-3$ & $\mathrm{CH}_{3}\left(\mathrm{CH}_{2}\right)_{3} \mathrm{CHO}$ & 1 & NA & $\mathrm{NA}$ \\
\hline 36 & isovaleraldehyde & $590-86-3$ & $\left(\mathrm{CH}_{3}\right)_{2} \mathrm{CHCH}_{2} \mathrm{CHO}$ & 1 & NA & NA \\
\hline 37 & decyl aldehyde & $112-31-2$ & $\mathrm{CH}_{3}\left(\mathrm{CH}_{2}\right)_{8} \mathrm{CHO}$ & 1 & NA & NA \\
\hline 38 & lauric aldehyde & $112-54-9$ & $\mathrm{CH}_{3}\left(\mathrm{CH}_{2}\right)_{10} \mathrm{CHO}$ & 1 & NA & NA \\
\hline 39 & Acetaldehyde & $75-07-0$ & $\mathrm{CH}_{3} \mathrm{CHO}$ & 1 & NA & NA \\
\hline 40 & propionaldehyde & $123-38-6$ & $\mathrm{CH}_{3} \mathrm{CH}_{2} \mathrm{CHO}$ & 1 & NA & NA \\
\hline 41 & butyraldehyde & $123-72-8$ & $\mathrm{CH}_{3}\left(\mathrm{CH}_{2}\right)_{2} \mathrm{CHO}$ & 1 & NA & NA \\
\hline 42 & hexanal & $66-25-1$ & $\mathrm{CH}_{3}\left(\mathrm{CH}_{2}\right)_{4} \mathrm{CHO}$ & 1 & NA & NA \\
\hline 43 & heptanal & $111-71-7$ & $\mathrm{CH}_{3}\left(\mathrm{CH}_{2}\right)_{5} \mathrm{CHO}$ & 1 & NA & NA \\
\hline 44 & octanal & $124-13-0$ & $\mathrm{CH}_{3}\left(\mathrm{CH}_{2}\right)_{6} \mathrm{CHO}$ & 1 & NA & NA \\
\hline 45 & nonanal & $124-13-0$ & $\mathrm{CH}_{3}\left(\mathrm{CH}_{2}\right)_{7} \mathrm{CHO}$ & 1 & NA & NA \\
\hline 46 & 2-methyl hexaldehyde & $925-54-2$ & $\mathrm{CH}_{3}\left(\mathrm{CH}_{2}\right)_{3} \mathrm{CH}\left(\mathrm{CH}_{3}\right) \mathrm{CHO}$ & 1 & NA & NA \\
\hline 47 & 3-methyl hexaldehyde & $19269-28-4$ & $\mathrm{CH}_{3}\left(\mathrm{CH}_{2}\right)_{2} \mathrm{CH}\left(\mathrm{CH}_{3}\right) \mathrm{CH}_{2} \mathrm{CHO}$ & 1 & NA & NA \\
\hline 48 & 2-ethylhexaldehyde & $123-05-7$ & $\mathrm{CH}_{3}\left(\mathrm{CH}_{2}\right)_{3} \mathrm{CH}\left(\mathrm{C}_{2} \mathrm{H}_{5}\right) \mathrm{CHO}$ & 1 & NA & NA \\
\hline 49 & Formic acid & $64-18-6$ & $\mathrm{HCOOH}$ & 1 & NA & NA \\
\hline 50 & Acetic acid & $64-19-7$ & $\mathrm{CH}_{3} \mathrm{COOH}$ & 1 & NA & NA \\
\hline
\end{tabular}




\begin{tabular}{|c|c|c|c|c|c|c|}
\hline 51 & Propionic acid & $79-09-4$ & $\mathrm{CH}_{3} \mathrm{CH}_{2} \mathrm{COOH}$ & 1 & NA & $\mathrm{NA}$ \\
\hline 52 & valeric acid & $109-52-4$ & $\mathrm{CH}_{3}\left(\mathrm{CH}_{2}\right)_{3} \mathrm{COOH}$ & 1 & NA & NA \\
\hline 53 & caproic acid & $142-62-1$ & $\mathrm{CH}_{3}\left(\mathrm{CH}_{2}\right)_{4} \mathrm{COOH}$ & 1 & NA & $\mathrm{NA}$ \\
\hline 54 & enanthic acid & $111-14-8$ & $\mathrm{CH}_{3}\left(\mathrm{CH}_{2}\right)_{5} \mathrm{COOH}$ & 1 & NA & $\mathrm{NA}$ \\
\hline 55 & capric acid & $334-48-5$ & $\mathrm{CH}_{3}\left(\mathrm{CH}_{2}\right)_{8} \mathrm{COOH}$ & 1 & NA & NA \\
\hline 56 & Undecanoic acid & $112-37-8$ & $\mathrm{CH}_{3}\left(\mathrm{CH}_{2}\right)_{9} \mathrm{COOH}$ & 1 & NA & NA \\
\hline 57 & lauric acid & $143-07-7$ & $\mathrm{CH}_{3}\left(\mathrm{CH}_{2}\right)_{10} \mathrm{COOH}$ & 1 & NA & NA \\
\hline 58 & myristic acid & $544-63-8$ & $\mathrm{CH}_{3}\left(\mathrm{CH}_{2}\right)_{12} \mathrm{COOH}$ & 1 & NA & NA \\
\hline 59 & palmitic acid & $57-10-3$ & $\mathrm{CH}_{3}\left(\mathrm{CH}_{2}\right)_{14} \mathrm{COOH}$ & 1 & NA & NA \\
\hline 60 & heptadecanoic acid & $506-12-7$ & $\mathrm{CH}_{3}\left(\mathrm{CH}_{2}\right)_{15} \mathrm{COOH}$ & 1 & NA & NA \\
\hline 61 & stearic acid & $57-11-4$ & $\mathrm{CH}_{3}\left(\mathrm{CH}_{2}\right)_{16} \mathrm{COOH}$ & 1 & NA & $\mathrm{NA}$ \\
\hline 62 & caprylic acid & $124-07-2$ & $\mathrm{CH}_{3}\left(\mathrm{CH}_{2}\right)_{6} \mathrm{COOH}$ & 1 & NA & NA \\
\hline 63 & nonanoic acid & $112-05-0$ & $\mathrm{CH}_{3}\left(\mathrm{CH}_{2}\right)_{7} \mathrm{COOH}$ & 1 & NA & $\mathrm{NA}$ \\
\hline 64 & Methylethylketone & $78-93-3$ & $\mathrm{CH}_{3} \mathrm{CH}_{2} \mathrm{COCH}_{3}$ & 1 & NA & NA \\
\hline 65 & 2-pentanone & $107-87-9$ & $\mathrm{CH}_{3}\left(\mathrm{CH}_{2}\right)_{2} \mathrm{COCH}_{3}$ & 1 & NA & NA \\
\hline 66 & diethyl ketone & $96-22-0$ & $\mathrm{CH}_{3} \mathrm{CH}_{2} \mathrm{OCH}_{2} \mathrm{CH}_{3}$ & 1 & NA & NA \\
\hline 67 & 2-hexanone & $591-78-6$ & $\mathrm{CH}_{3}\left(\mathrm{CH}_{2}\right)_{3} \mathrm{COCH}_{3}$ & 1 & NA & NA \\
\hline 68 & 4-heptanone & $123-19-3$ & $\mathrm{CH}_{3}\left(\mathrm{CH}_{2}\right)_{2} \mathrm{CO}\left(\mathrm{CH}_{2}\right)_{2} \mathrm{CH}_{3}$ & 1 & NA & NA \\
\hline 69 & 3-heptanone & $106-35-4$ & $\mathrm{CH}_{3}\left(\mathrm{CH}_{2}\right)_{3} \mathrm{COC}_{2} \mathrm{H}_{5}$ & 1 & NA & NA \\
\hline 70 & diamyl ketone & $927-49-1$ & $\mathrm{CH}_{3}\left(\mathrm{CH}_{2}\right)_{4} \mathrm{CO}\left(\mathrm{CH}_{2}\right)_{4} \mathrm{CH}_{3}$ & 1 & NA & NA \\
\hline 71 & di-n-hexyl ketone & $462-18-0$ & $\mathrm{CH}_{3}\left(\mathrm{CH}_{2}\right)_{5} \mathrm{CO}\left(\mathrm{CH}_{2}\right)_{5} \mathrm{CH}_{3}$ & 1 & NA & NA \\
\hline 72 & 2-octanone & $111-13-7$ & $\mathrm{CH}_{3}\left(\mathrm{CH}_{2}\right)_{5} \mathrm{COCH}_{3}$ & 1 & NA & NA \\
\hline 73 & acetone & $67-64-1$ & $\mathrm{CH}_{3} \mathrm{COCH}_{3}$ & 1 & NA & NA \\
\hline 74 & Methyl isopropyl ketone & $563-80-4$ & $\left(\mathrm{CH}_{3}\right)_{2} \mathrm{CHCOCH}_{3}$ & 1 & NA & NA \\
\hline 75 & Methyl isobutyl ketone & $108-10-1$ & $\left(\mathrm{CH}_{3}\right)_{2} \mathrm{CHCH}_{2} \mathrm{COCH}_{3}$ & 1 & NA & NA \\
\hline 76 & Ethyl formate & $109-94-4$ & $\mathrm{HCOOCH}_{2} \mathrm{CH}_{3}$ & 1 & NA & NA \\
\hline 77 & Methyl propionate & $554-12-1$ & $\mathrm{CH}_{3} \mathrm{CH}_{2} \mathrm{COOCH}_{3}$ & 1 & NA & NA \\
\hline 78 & ethyl acetate & $141-78-6$ & $\mathrm{CH}_{3} \mathrm{COOCH}_{2} \mathrm{CH}_{3}$ & 1 & NA & NA \\
\hline
\end{tabular}




\begin{tabular}{|c|c|c|c|c|c|c|}
\hline 79 & methyl butyrate & $623-42-7$ & $\mathrm{CH}_{3}\left(\mathrm{CH}_{2}\right)_{2} \mathrm{COOCH}_{3}$ & 1 & NA & $\mathrm{NA}$ \\
\hline 80 & ethyl propionate & $105-37-3$ & $\mathrm{C}_{2} \mathrm{H}_{5} \mathrm{COOC}_{2} \mathrm{H}_{5}$ & 1 & NA & NA \\
\hline 81 & butyl formate & $592-84-7$ & $\mathrm{HCOO}\left(\mathrm{CH}_{2}\right)_{3} \mathrm{CH}_{3}$ & 1 & NA & NA \\
\hline 82 & propyl acetate & $109-60-4$ & $\mathrm{CH}_{3} \mathrm{COO}\left(\mathrm{CH}_{2}\right)_{2} \mathrm{CH}_{3}$ & 1 & NA & $\mathrm{NA}$ \\
\hline 83 & methyl valerate & $624-24-8$ & $\mathrm{CH}_{3}\left(\mathrm{CH}_{2}\right)_{3} \mathrm{COOCH}_{3}$ & 1 & NA & NA \\
\hline 84 & butyl acetate & $123-86-4$ & $\mathrm{CH}_{3} \mathrm{COO}\left(\mathrm{CH}_{2}\right)_{3} \mathrm{CH}_{3}$ & 1 & NA & NA \\
\hline 85 & isobutyl acetate & $110-19-0$ & $\mathrm{CH}_{3} \mathrm{COOCH}_{2} \mathrm{CH}\left(\mathrm{CH}_{3}\right)_{2}$ & 1 & NA & NA \\
\hline 86 & amyl formate & $638-49-3$ & $\mathrm{HCOO}\left(\mathrm{CH}_{2}\right)_{4} \mathrm{CH}_{3}$ & 1 & NA & NA \\
\hline 87 & ethyl butyrate & $105-54-4$ & $\mathrm{CH}_{3}\left(\mathrm{CH}_{2}\right)_{2} \mathrm{COOC}_{2} \mathrm{H}_{5}$ & 1 & NA & NA \\
\hline 88 & n-propyl propionate & $106-36-5$ & $\mathrm{C}_{2} \mathrm{H}_{5} \mathrm{COO}\left(\mathrm{CH}_{2}\right)_{2} \mathrm{CH}_{3}$ & 1 & NA & NA \\
\hline 89 & methyl capronate & $106-70-7$ & $\mathrm{CH}_{3}\left(\mathrm{CH}_{2}\right)_{4} \mathrm{COOCH}_{3}$ & 1 & NA & $\mathrm{NA}$ \\
\hline 90 & n-amyl acetate & $628-63-7$ & $\mathrm{CH}_{3} \mathrm{COO}\left(\mathrm{CH}_{2}\right)_{4} \mathrm{CH}_{3}$ & 1 & NA & NA \\
\hline 91 & n-butyl propionate & $590-01-2$ & $\mathrm{C}_{2} \mathrm{H}_{5} \mathrm{COO}\left(\mathrm{CH}_{2}\right)_{3} \mathrm{CH}_{3}$ & 1 & NA & NA \\
\hline 92 & isoamyl acetate & $123-92-2$ & $\mathrm{CH}_{3} \mathrm{COO}\left(\mathrm{CH}_{2}\right)_{2} \mathrm{CH}\left(\mathrm{CH}_{3}\right)_{2}$ & 1 & NA & NA \\
\hline 93 & n-hexyl formate & $629-33-4$ & $\mathrm{HCOO}\left(\mathrm{CH}_{2}\right)_{5} \mathrm{CH}_{3}$ & 1 & NA & NA \\
\hline 94 & n-hexyl butyrate & $2639-63-6$ & $\mathrm{CH}_{3}\left(\mathrm{CH}_{2}\right)_{2} \mathrm{COO}\left(\mathrm{CH}_{2}\right)_{5} \mathrm{CH}_{3}$ & 1 & NA & NA \\
\hline 95 & n-heptyl propionate & $2216-81-1$ & $\mathrm{C}_{2} \mathrm{H}_{5} \mathrm{COO}\left(\mathrm{CH}_{2}\right)_{6} \mathrm{CH}_{3}$ & 1 & NA & NA \\
\hline 96 & n-octyl acetate & $112-14-1$ & $\mathrm{CH}_{3} \mathrm{COO}\left(\mathrm{CH}_{2}\right)_{7} \mathrm{CH}_{3}$ & 1 & NA & NA \\
\hline 97 & n-octyl propionate & $142-60-9$ & $\mathrm{C}_{2} \mathrm{H}_{5} \mathrm{COO}\left(\mathrm{CH}_{2}\right)_{7} \mathrm{CH}_{3}$ & 1 & NA & NA \\
\hline 98 & heptylbutanoate & $5870-93-9$ & $\mathrm{CH}_{3}\left(\mathrm{CH}_{2}\right)_{2} \mathrm{COO}\left(\mathrm{CH}_{2}\right)_{6} \mathrm{CH}_{3}$ & 1 & NA & NA \\
\hline 99 & n-decyl acetate & $112-17-4$ & $\mathrm{CH}_{3} \mathrm{COO}\left(\mathrm{CH}_{2}\right)_{9} \mathrm{CH}_{3}$ & 1 & NA & NA \\
\hline 100 & n-octyl butyrate & $110-39-4$ & $\mathrm{CH}_{3}\left(\mathrm{CH}_{2}\right)_{2} \mathrm{COO}\left(\mathrm{CH}_{2}\right)_{7} \mathrm{CH}_{3}$ & 1 & NA & NA \\
\hline 101 & methyl stearate & $112-61-8$ & $\mathrm{CH}_{3}\left(\mathrm{CH}_{2}\right)_{16} \mathrm{COOCH}_{3}$ & 1 & NA & NA \\
\hline 102 & n-octadecyl acetate & $822-23-1$ & $\mathrm{CH}_{3} \mathrm{COO}\left(\mathrm{CH}_{2}\right)_{17} \mathrm{CH}_{3}$ & 1 & NA & NA \\
\hline 103 & n-butyl stearate & $123-95-5$ & $\mathrm{CH}_{3}\left(\mathrm{CH}_{2}\right)_{16} \mathrm{COO}\left(\mathrm{CH}_{2}\right)_{3} \mathrm{CH}_{3}$ & 1 & NA & NA \\
\hline 104 & n-amyl propionate & $624-54-4$ & $\mathrm{C}_{2} \mathrm{H}_{5} \mathrm{COO}\left(\mathrm{CH}_{2}\right)_{4} \mathrm{CH}_{3}$ & 1 & NA & NA \\
\hline 105 & n-hexyl acetate & $142-92-7$ & $\mathrm{CH}_{3} \mathrm{COO}\left(\mathrm{CH}_{2}\right)_{5} \mathrm{CH}_{3}$ & 1 & NA & NA \\
\hline 106 & n-heptyl formate & $112-23-2$ & $\mathrm{HCOO}\left(\mathrm{CH}_{2}\right)_{6} \mathrm{CH}_{3}$ & 1 & NA & NA \\
\hline
\end{tabular}




\begin{tabular}{|c|c|c|c|c|c|c|}
\hline 107 & isoamyl propionate & $105-68-0$ & $\mathrm{C}_{2} \mathrm{H}_{5} \mathrm{COO}\left(\mathrm{CH}_{2}\right)_{2} \mathrm{CH}\left(\mathrm{CH}_{3}\right)_{2}$ & 1 & $\mathrm{NA}$ & NA \\
\hline 108 & Ethyl hexanoate & $123-66-0$ & $\mathrm{CH}_{3}\left(\mathrm{CH}_{2}\right)_{4} \mathrm{COOC}_{2} \mathrm{H}_{5}$ & 1 & NA & NA \\
\hline 109 & n-amylbutyrate & $540-18-1$ & $\mathrm{CH}_{3}\left(\mathrm{CH}_{2}\right)_{2} \mathrm{COO}\left(\mathrm{CH}_{2}\right)_{4} \mathrm{CH}_{3}$ & 1 & NA & NA \\
\hline 110 & n-hexyl propionate & $2445-76-3$ & $\mathrm{C}_{2} \mathrm{H}_{5} \mathrm{COO}\left(\mathrm{CH}_{2}\right)_{5} \mathrm{CH}_{3}$ & 1 & NA & NA \\
\hline 111 & n-octyl formate & $112-32-3$ & $\mathrm{HCOO}\left(\mathrm{CH}_{2}\right)_{7} \mathrm{CH}_{3}$ & 1 & NA & NA \\
\hline 112 & n-heptylacetate & $112-06-1$ & $\mathrm{CH}_{3} \mathrm{COO}\left(\mathrm{CH}_{2}\right)_{6} \mathrm{CH}_{3}$ & 1 & NA & NA \\
\hline 113 & Undecylic aldehyde* & $112-44-7$ & $\mathrm{CH}_{3}\left(\mathrm{CH}_{2}\right)_{9} \mathrm{CHO}$ & NA & $\geq 97$ & Aladdin Reagent Co., Ltd. \\
\hline 114 & Dodecyl aldehyde* & $112-54-9$ & $\mathrm{CH}_{3}\left(\mathrm{CH}_{2}\right)_{10} \mathrm{CHO}$ & NA & $\geq 95$ & Aladdin Reagent Co., Ltd. \\
\hline 115 & 2-Heptanone* & $110-43-0$ & $\mathrm{CH}_{3} \mathrm{CO}\left(\mathrm{CH}_{2}\right)_{4} \mathrm{CH}_{3}$ & NA & $\geq 99$ & Aladdin Reagent Co., Ltd. \\
\hline 116 & 2-Nonanone* & $821-55-6$ & $\mathrm{CH}_{3} \mathrm{CO}\left(\mathrm{CH}_{2}\right)_{6} \mathrm{CH}_{3}$ & NA & $\geq 99$ & Aladdin Reagent Co., Ltd. \\
\hline 117 & 2-Undecanone* & $112-12-9$ & $\mathrm{CH}_{3} \mathrm{CO}\left(\mathrm{CH}_{2}\right)_{8} \mathrm{CH}_{3}$ & NA & $\geq 99$ & Aladdin Reagent Co., Ltd. \\
\hline 118 & n-Butyric acid* & $107-92-6$ & $\mathrm{CH}_{3}\left(\mathrm{CH}_{2}\right)_{2} \mathrm{COOH}$ & NA & $\geq 99$ & Aladdin Reagent Co., Ltd. \\
\hline 119 & 3,5,5-Trimethylhexanoic acid* & $3302-10-1$ & $\left(\mathrm{CH}_{3}\right)_{3} \mathrm{CCH}_{2} \mathrm{CH}\left(\mathrm{CH}_{3}\right) \mathrm{CH}_{2} \mathrm{COOH}$ & NA & $\geq 97$ & Aladdin Reagent Co., Ltd. \\
\hline 120 & 2-Methylbutyric Acid* & $116-53-0$ & $\mathrm{C}_{2} \mathrm{H}_{5} \mathrm{CH}\left(\mathrm{CH}_{3}\right) \mathrm{COOH}$ & NA & $\geq 98$ & Aladdin Reagent Co., Ltd. \\
\hline 121 & 2,6-dimethyl-2-heptanol* & $13254-34-7$ & $\left(\mathrm{CH}_{3}\right)_{2} \mathrm{CH}\left(\mathrm{CH}_{2}\right)_{3} \mathrm{CH}\left(\mathrm{CH}_{3}\right) \mathrm{CH}_{2} \mathrm{OH}$ & NA & $\geq 99$ & Aladdin Reagent Co., Ltd. \\
\hline 122 & 3,5,5-trimethyl-1-hexanol* & $3452-97-9$ & $\left(\mathrm{CH}_{3}\right)_{3} \mathrm{CCH}_{2} \mathrm{CH}\left(\mathrm{CH}_{3}\right)\left(\mathrm{CH}_{2}\right)_{2} \mathrm{OH}$ & NA & $\geq 99$ & Aladdin Reagent Co., Ltd. \\
\hline 123 & 4-Methyl-2-pentanol* & $108-11-2$ & $\left(\mathrm{CH}_{3}\right)_{2} \mathrm{CHCH}_{2} \mathrm{CH}\left(\mathrm{CH}_{3}\right) \mathrm{OH}$ & NA & $\geq 99$ & Aladdin Reagent Co., Ltd. \\
\hline 124 & Isoamyl acetate* & $123-92-2$ & $\mathrm{CH}_{3} \mathrm{COO}\left(\mathrm{CH}_{2}\right)_{2} \mathrm{CH}\left(\mathrm{CH}_{3}\right)_{2}$ & NA & $\geq 99$ & Aladdin Reagent Co., Ltd. \\
\hline 125 & isobutyl formate* & $542-55-2$ & $\mathrm{HCOOCH}_{2} \mathrm{CH}\left(\mathrm{CH}_{3}\right)_{2}$ & NA & $\geq 95$ & Aladdin Reagent Co., Ltd. \\
\hline 126 & Ethyl tert-amyl ether* & 919-94-8 & $\mathrm{C}_{2} \mathrm{H}_{5} \mathrm{OC}\left(\mathrm{CH}_{3}\right)_{2} \mathrm{C}_{2} \mathrm{H}_{5}$ & NA & $\geq 98$ & Aladdin Reagent Co., Ltd. \\
\hline 127 & Methyl isopropyl ether* & $598-53-8$ & $\mathrm{CH}_{3} \mathrm{OCH}\left(\mathrm{CH}_{3}\right)_{2}$ & NA & $\geq 98$ & Aladdin Reagent Co., Ltd. \\
\hline
\end{tabular}

Table S2. Experiment and calculated values of thermal conductivity for training set, test set and prediction set

\begin{tabular}{|c|c|c|c|c|c|c|c|c|c|c|c|c|}
\hline Set & No. & Name & $S M 2 \_B(s)$ & SICO & IC1 & $T$ & $E t a_{-} F$ & $M A T S 2 m$ & $\begin{array}{c}\lambda_{\text {exp }} / \\
(\mathbf{W} / \mathbf{m} \cdot \mathbf{K})\end{array}$ & $\begin{array}{c}\lambda_{\text {cal }} / \\
(\mathbf{W} / \mathbf{m} \cdot \mathbf{K})\end{array}$ & Residual & $\begin{array}{c}\text { Prediction } \\
\text { Quality }\end{array}$ \\
\hline Training set & 1 & Methane & 3.771 & 0.484 & 1.792 & 200 & 0.456 & -0.408 & 0.236 & 0.218 & 0.018 & I \\
\hline
\end{tabular}




\begin{tabular}{|c|c|c|c|c|c|c|c|c|c|c|c|}
\hline 2 & Methane & 3.771 & 0.484 & 1.792 & 280 & 0.456 & -0.408 & 0.208 & 0.202 & 0.006 & I \\
\hline 3 & Methane & 3.771 & 0.484 & 1.792 & 300 & 0.456 & -0.408 & 0.202 & 0.198 & 0.004 & / \\
\hline 4 & Methane & 3.771 & 0.484 & 1.792 & 380 & 0.456 & -0.408 & 0.180 & 0.182 & -0.002 & I \\
\hline 5 & Methane & 3.771 & 0.484 & 1.792 & 400 & 0.456 & -0.408 & 0.176 & 0.178 & -0.002 & I \\
\hline 6 & Methane & 3.771 & 0.484 & 1.792 & 440 & 0.456 & -0.408 & 0.168 & 0.170 & -0.002 & I \\
\hline 7 & Methane & 3.771 & 0.484 & 1.792 & 480 & 0.456 & -0.408 & 0.162 & 0.162 & 0.000 & I \\
\hline 8 & Ethanol & 3.873 & 0.386 & 1.880 & 160 & 0.527 & -0.257 & 0.207 & 0.190 & 0.017 & I \\
\hline 9 & Ethanol & 3.873 & 0.386 & 1.880 & 180 & 0.527 & -0.257 & 0.202 & 0.186 & 0.016 & / \\
\hline 10 & Ethanol & 3.873 & 0.386 & 1.880 & 200 & 0.527 & -0.257 & 0.196 & 0.182 & 0.014 & / \\
\hline 11 & Ethanol & 3.873 & 0.386 & 1.880 & 220 & 0.527 & -0.257 & 0.189 & 0.178 & 0.011 & I \\
\hline 12 & Ethanol & 3.873 & 0.386 & 1.880 & 240 & 0.527 & -0.257 & 0.183 & 0.174 & 0.009 & I \\
\hline 13 & Ethanol & 3.873 & 0.386 & 1.880 & 260 & 0.527 & -0.257 & 0.178 & 0.170 & 0.008 & I \\
\hline 14 & Ethanol & 3.873 & 0.386 & 1.880 & 280 & 0.527 & -0.257 & 0.173 & 0.166 & 0.007 & / \\
\hline 15 & Ethanol & 3.873 & 0.386 & 1.880 & 300 & 0.527 & -0.257 & 0.166 & 0.162 & 0.004 & / \\
\hline 16 & Ethanol & 3.873 & 0.386 & 1.880 & 380 & 0.527 & -0.257 & 0.147 & 0.146 & 0.001 & / \\
\hline 17 & n-Propyl alcohol & 3.958 & 0.332 & 1.947 & 160 & 0.605 & -0.258 & 0.182 & 0.176 & 0.006 & 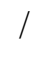 \\
\hline 18 & n-Propyl alcohol & 3.958 & 0.332 & 1.947 & 220 & 0.605 & -0.258 & 0.172 & 0.164 & 0.008 & / \\
\hline 19 & n-Propyl alcohol & 3.958 & 0.332 & 1.947 & 240 & 0.605 & -0.258 & 0.168 & 0.160 & 0.008 & 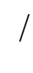 \\
\hline 20 & n-Propyl alcohol & 3.958 & 0.332 & 1.947 & 260 & 0.605 & -0.258 & 0.163 & 0.156 & 0.007 & I \\
\hline 21 & n-Propyl alcohol & 3.958 & 0.332 & 1.947 & 280 & 0.605 & -0.258 & 0.158 & 0.152 & 0.006 & I \\
\hline 22 & n-Propyl alcohol & 3.958 & 0.332 & 1.947 & 300 & 0.605 & -0.258 & 0.154 & 0.148 & 0.006 & I \\
\hline 23 & n-Propyl alcohol & 3.958 & 0.332 & 1.947 & 320 & 0.605 & -0.258 & 0.150 & 0.144 & 0.006 & l \\
\hline
\end{tabular}




\begin{tabular}{|c|c|c|c|c|c|c|c|c|c|c|c|}
\hline 24 & n-Propyl alcohol & 3.958 & 0.332 & 1.947 & 380 & 0.605 & -0.258 & 0.139 & 0.131 & 0.008 & I \\
\hline 25 & n-Propyl alcohol & 3.958 & 0.332 & 1.947 & 400 & 0.605 & -0.258 & 0.136 & 0.127 & 0.009 & I \\
\hline 26 & n-Propyl alcohol & 3.958 & 0.332 & 1.947 & 420 & 0.605 & -0.258 & 0.132 & 0.123 & 0.009 & I \\
\hline 27 & n-Propyl alcohol & 3.958 & 0.332 & 1.947 & 440 & 0.605 & -0.258 & 0.129 & 0.119 & 0.010 & / \\
\hline 28 & n-Propyl alcohol & 3.958 & 0.332 & 1.947 & 480 & 0.605 & -0.258 & 0.124 & 0.111 & 0.013 & I \\
\hline 29 & n-Propyl alcohol & 3.958 & 0.332 & 1.947 & 370 & 0.605 & -0.258 & 0.141 & 0.133 & 0.008 & / \\
\hline 30 & Isopropyl alcohol & 3.990 & 0.332 & 1.781 & 220 & 0.640 & 0.005 & 0.155 & 0.159 & -0.004 & / \\
\hline 31 & Isopropyl alcohol & 3.990 & 0.332 & 1.781 & 240 & 0.640 & 0.005 & 0.150 & 0.155 & -0.005 & 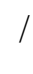 \\
\hline 32 & Isopropyl alcohol & 3.990 & 0.332 & 1.781 & 280 & 0.640 & 0.005 & 0.142 & 0.147 & -0.005 & r \\
\hline 33 & Isopropyl alcohol & 3.990 & 0.332 & 1.781 & 320 & 0.640 & 0.005 & 0.134 & 0.138 & -0.004 & I \\
\hline 34 & Isopropyl alcohol & 3.990 & 0.332 & 1.781 & 340 & 0.640 & 0.005 & 0.131 & 0.134 & -0.003 & / \\
\hline 35 & Isopropyl alcohol & 3.990 & 0.332 & 1.781 & 360 & 0.640 & 0.005 & 0.128 & 0.130 & -0.002 & / \\
\hline 36 & Isopropyl alcohol & 3.990 & 0.332 & 1.781 & 400 & 0.640 & 0.005 & 0.122 & 0.122 & 0.000 & / \\
\hline 37 & Isopropyl alcohol & 3.990 & 0.332 & 1.781 & 420 & 0.640 & 0.005 & 0.119 & 0.118 & 0.001 & 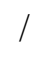 \\
\hline 38 & Isopropyl alcohol & 3.990 & 0.332 & 1.781 & 440 & 0.640 & 0.005 & 0.116 & 0.114 & 0.002 & 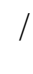 \\
\hline 39 & Isopropyl alcohol & 3.990 & 0.332 & 1.781 & 460 & 0.640 & 0.005 & 0.113 & 0.110 & 0.003 & 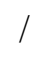 \\
\hline 40 & Isopropyl alcohol & 3.990 & 0.332 & 1.781 & 470 & 0.640 & 0.005 & 0.112 & 0.108 & 0.004 & 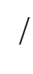 \\
\hline 41 & Isopropyl alcohol & 3.990 & 0.332 & 1.781 & 350 & 0.640 & 0.005 & 0.129 & 0.132 & -0.003 & 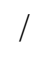 \\
\hline 42 & n-butanol & 4.036 & 0.297 & 1.872 & 290 & 0.669 & -0.258 & 0.153 & 0.148 & 0.005 & 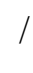 \\
\hline 43 & n-butanol & 4.036 & 0.297 & 1.872 & 300 & 0.669 & -0.258 & 0.151 & 0.146 & 0.005 & I \\
\hline 44 & n-butanol & 4.036 & 0.297 & 1.872 & 320 & 0.669 & -0.258 & 0.147 & 0.142 & 0.005 & 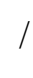 \\
\hline 45 & n-butanol & 4.036 & 0.297 & 1.872 & 340 & 0.669 & -0.258 & 0.143 & 0.138 & 0.005 & / \\
\hline
\end{tabular}




\begin{tabular}{|c|c|c|c|c|c|c|c|c|c|c|c|}
\hline 46 & n-butanol & 4.036 & 0.297 & 1.872 & 360 & 0.669 & -0.258 & 0.139 & 0.134 & 0.005 & I \\
\hline 47 & isobutyl alcohol & 4.065 & 0.297 & 1.872 & 180 & 0.722 & -0.106 & 0.152 & 0.163 & -0.011 & / \\
\hline 48 & isobutyl alcohol & 4.065 & 0.297 & 1.872 & 200 & 0.722 & -0.106 & 0.149 & 0.159 & -0.010 & I \\
\hline 49 & isobutyl alcohol & 4.065 & 0.297 & 1.872 & 240 & 0.722 & -0.106 & 0.143 & 0.150 & -0.007 & I \\
\hline 50 & isobutyl alcohol & 4.065 & 0.297 & 1.872 & 260 & 0.722 & -0.106 & 0.140 & 0.146 & -0.006 & I \\
\hline 51 & isobutyl alcohol & 4.065 & 0.297 & 1.872 & 320 & 0.722 & -0.106 & 0.130 & 0.134 & -0.004 & / \\
\hline 52 & isobutyl alcohol & 4.065 & 0.297 & 1.872 & 340 & 0.722 & -0.106 & 0.128 & 0.130 & -0.002 & / \\
\hline 53 & isobutyl alcohol & 4.065 & 0.297 & 1.872 & 360 & 0.722 & -0.106 & 0.125 & 0.126 & -0.001 & t \\
\hline 54 & isobutyl alcohol & 4.065 & 0.297 & 1.872 & 380 & 0.722 & -0.106 & 0.122 & 0.122 & 0.000 & t \\
\hline 55 & isobutyl alcohol & 4.065 & 0.297 & 1.872 & 400 & 0.722 & -0.106 & 0.120 & 0.118 & 0.002 & I \\
\hline 56 & isobutyl alcohol & 4.065 & 0.297 & 1.872 & 420 & 0.722 & -0.106 & 0.118 & 0.114 & 0.004 & I \\
\hline 57 & isobutyl alcohol & 4.065 & 0.297 & 1.872 & 440 & 0.722 & -0.106 & 0.115 & 0.110 & 0.005 & I \\
\hline 58 & isobutyl alcohol & 4.065 & 0.297 & 1.872 & 460 & 0.722 & -0.106 & 0.113 & 0.106 & 0.007 & / \\
\hline 59 & isobutyl alcohol & 4.065 & 0.297 & 1.872 & 470 & 0.722 & -0.106 & 0.112 & 0.104 & 0.008 & / \\
\hline 60 & tertbutyl alcohol & 4.098 & 0.297 & 1.688 & 300 & 0.772 & 0.309 & 0.112 & 0.125 & -0.013 & I \\
\hline 61 & tertbutyl alcohol & 4.098 & 0.297 & 1.688 & 310 & 0.772 & 0.309 & 0.111 & 0.123 & -0.012 & t \\
\hline 62 & tertbutyl alcohol & 4.098 & 0.297 & 1.688 & 340 & 0.772 & 0.309 & 0.110 & 0.117 & -0.007 & 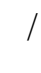 \\
\hline 63 & tertbutyl alcohol & 4.098 & 0.297 & 1.688 & 360 & 0.772 & 0.309 & 0.108 & 0.113 & -0.005 & t \\
\hline 64 & tertbutyl alcohol & 4.098 & 0.297 & 1.688 & 380 & 0.772 & 0.309 & 0.107 & 0.109 & -0.002 & $t$ \\
\hline 65 & tertbutyl alcohol & 4.098 & 0.297 & 1.688 & 390 & 0.772 & 0.309 & 0.106 & 0.107 & -0.001 & I \\
\hline 66 & tertbutyl alcohol & 4.098 & 0.297 & 1.688 & 400 & 0.772 & 0.309 & 0.105 & 0.105 & 0.000 & 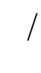 \\
\hline 67 & tertbutyl alcohol & 4.098 & 0.297 & 1.688 & 420 & 0.772 & 0.309 & 0.104 & 0.101 & 0.003 & I \\
\hline
\end{tabular}




\begin{tabular}{|c|c|c|c|c|c|c|c|c|c|c|c|}
\hline 68 & tertbutyl alcohol & 4.098 & 0.297 & 1.688 & 430 & 0.772 & 0.309 & 0.102 & 0.099 & 0.003 & I \\
\hline 69 & tertbutyl alcohol & 4.098 & 0.297 & 1.688 & 440 & 0.772 & 0.309 & 0.102 & 0.097 & 0.005 & / \\
\hline 70 & tertbutyl alcohol & 4.098 & 0.297 & 1.688 & 450 & 0.772 & 0.309 & 0.102 & 0.095 & 0.007 & I \\
\hline 71 & n-amyl alcohol & 4.108 & 0.272 & 1.792 & 200 & 0.723 & -0.258 & 0.168 & 0.167 & 0.001 & I \\
\hline 72 & n-amyl alcohol & 4.108 & 0.272 & 1.792 & 220 & 0.723 & -0.258 & 0.165 & 0.163 & 0.002 & I \\
\hline 73 & n-amyl alcohol & 4.108 & 0.272 & 1.792 & 240 & 0.723 & -0.258 & 0.162 & 0.159 & 0.003 & / \\
\hline 74 & n-amyl alcohol & 4.108 & 0.272 & 1.792 & 260 & 0.723 & -0.258 & 0.158 & 0.155 & 0.003 & / \\
\hline 75 & n-amyl alcohol & 4.108 & 0.272 & 1.792 & 280 & 0.723 & -0.258 & 0.154 & 0.151 & 0.003 & t \\
\hline 76 & n-amyl alcohol & 4.108 & 0.272 & 1.792 & 290 & 0.723 & -0.258 & 0.152 & 0.149 & 0.003 & t \\
\hline 77 & n-amyl alcohol & 4.108 & 0.272 & 1.792 & 300 & 0.723 & -0.258 & 0.150 & 0.147 & 0.003 & I \\
\hline 78 & n-amyl alcohol & 4.108 & 0.272 & 1.792 & 320 & 0.723 & -0.258 & 0.146 & 0.143 & 0.003 & I \\
\hline 79 & n-amyl alcohol & 4.108 & 0.272 & 1.792 & 340 & 0.723 & -0.258 & 0.142 & 0.139 & 0.003 & I \\
\hline 80 & n-amyl alcohol & 4.108 & 0.272 & 1.792 & 360 & 0.723 & -0.258 & 0.135 & 0.135 & 0.000 & / \\
\hline 81 & n-amyl alcohol & 4.108 & 0.272 & 1.792 & 380 & 0.723 & -0.258 & 0.135 & 0.131 & 0.004 & 1 \\
\hline 82 & n-amyl alcohol & 4.108 & 0.272 & 1.792 & 400 & 0.723 & -0.258 & 0.131 & 0.127 & 0.004 & 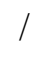 \\
\hline 83 & n-amyl alcohol & 4.108 & 0.272 & 1.792 & 440 & 0.723 & -0.258 & 0.125 & 0.119 & 0.006 & t \\
\hline 84 & n-amyl alcohol & 4.108 & 0.272 & 1.792 & 460 & 0.723 & -0.258 & 0.122 & 0.115 & 0.007 & 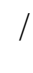 \\
\hline 85 & n-amyl alcohol & 4.108 & 0.272 & 1.792 & 480 & 0.723 & -0.258 & 0.118 & 0.111 & 0.007 & 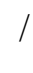 \\
\hline 86 & n-amyl alcohol & 4.108 & 0.272 & 1.792 & 500 & 0.723 & -0.258 & 0.115 & 0.106 & 0.009 & $t$ \\
\hline 87 & isoamyl alcohol & 4.136 & 0.272 & 1.945 & 180 & 0.758 & -0.132 & 0.150 & 0.158 & -0.008 & I \\
\hline 88 & isoamyl alcohol & 4.136 & 0.272 & 1.945 & 200 & 0.758 & -0.132 & 0.148 & 0.154 & -0.006 & 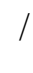 \\
\hline 89 & isoamyl alcohol & 4.136 & 0.272 & 1.945 & 240 & 0.758 & -0.132 & 0.143 & 0.145 & -0.002 & / \\
\hline
\end{tabular}




\begin{tabular}{|c|c|c|c|c|c|c|c|c|c|c|c|}
\hline 90 & isoamyl alcohol & 4.136 & 0.272 & 1.945 & 280 & 0.758 & -0.132 & 0.137 & 0.137 & 0.000 & I \\
\hline 91 & isoamyl alcohol & 4.136 & 0.272 & 1.945 & 320 & 0.758 & -0.132 & 0.132 & 0.129 & 0.003 & I \\
\hline 92 & isoamyl alcohol & 4.136 & 0.272 & 1.945 & 340 & 0.758 & -0.132 & 0.128 & 0.125 & 0.003 & I \\
\hline 93 & isoamyl alcohol & 4.136 & 0.272 & 1.945 & 360 & 0.758 & -0.132 & 0.126 & 0.121 & 0.005 & I \\
\hline 94 & isoamyl alcohol & 4.136 & 0.272 & 1.945 & 380 & 0.758 & -0.132 & 0.122 & 0.117 & 0.005 & I \\
\hline 95 & isoamyl alcohol & 4.136 & 0.272 & 1.945 & 400 & 0.758 & -0.132 & 0.118 & 0.113 & 0.005 & I \\
\hline 96 & isoamyl alcohol & 4.136 & 0.272 & 1.945 & 420 & 0.758 & -0.132 & 0.115 & 0.109 & 0.006 & / \\
\hline 97 & isoamyl alcohol & 4.136 & 0.272 & 1.945 & 460 & 0.758 & -0.132 & 0.108 & 0.101 & 0.007 & t \\
\hline 98 & isoamyl alcohol & 4.136 & 0.272 & 1.945 & 480 & 0.758 & -0.132 & 0.104 & 0.097 & 0.007 & / \\
\hline 99 & isoamyl alcohol & 4.136 & 0.272 & 1.945 & 500 & 0.758 & -0.132 & 0.100 & 0.093 & 0.007 & I \\
\hline 100 & isoamyl alcohol & 4.136 & 0.272 & 1.945 & 520 & 0.758 & -0.132 & 0.097 & 0.089 & 0.008 & I \\
\hline 101 & 1-hexanol & 4.176 & 0.254 & 1.721 & 240 & 0.768 & -0.257 & 0.162 & 0.162 & 0.000 & I \\
\hline 102 & 1-hexanol & 4.176 & 0.254 & 1.721 & 260 & 0.768 & -0.257 & 0.158 & 0.158 & 0.000 & / \\
\hline 103 & 1-hexanol & 4.176 & 0.254 & 1.721 & 280 & 0.768 & -0.257 & 0.156 & 0.153 & 0.003 & / \\
\hline 104 & 1-hexanol & 4.176 & 0.254 & 1.721 & 320 & 0.768 & -0.257 & 0.148 & 0.145 & 0.003 & / \\
\hline 105 & 1-hexanol & 4.176 & 0.254 & 1.721 & 340 & 0.768 & -0.257 & 0.144 & 0.141 & 0.003 & 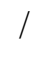 \\
\hline 106 & 1-hexanol & 4.176 & 0.254 & 1.721 & 360 & 0.768 & -0.257 & 0.140 & 0.137 & 0.003 & 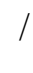 \\
\hline 107 & 1-hexanol & 4.176 & 0.254 & 1.721 & 380 & 0.768 & -0.257 & 0.136 & 0.133 & 0.003 & t \\
\hline 108 & 1-hexanol & 4.176 & 0.254 & 1.721 & 420 & 0.768 & -0.257 & 0.127 & 0.125 & 0.002 & I \\
\hline 109 & 1-hexanol & 4.176 & 0.254 & 1.721 & 440 & 0.768 & -0.257 & 0.122 & 0.121 & 0.001 & I \\
\hline 110 & 1-hexanol & 4.176 & 0.254 & 1.721 & 460 & 0.768 & -0.257 & 0.118 & 0.117 & 0.001 & , \\
\hline 111 & 1-hexanol & 4.176 & 0.254 & 1.721 & 500 & 0.768 & -0.257 & 0.108 & 0.109 & -0.001 & I \\
\hline
\end{tabular}




\begin{tabular}{|c|c|c|c|c|c|c|c|c|c|c|c|}
\hline 112 & 1-hexanol & 4.176 & 0.254 & 1.721 & 520 & 0.768 & -0.257 & 0.103 & 0.105 & -0.002 & I \\
\hline 113 & 1-hexanol & 4.176 & 0.254 & 1.721 & 560 & 0.768 & -0.257 & 0.094 & 0.097 & -0.003 & I \\
\hline 114 & 2-hexanol & 4.202 & 0.254 & 1.780 & 200 & 0.819 & -0.111 & 0.152 & 0.159 & -0.007 & I \\
\hline 115 & 2-hexanol & 4.202 & 0.254 & 1.780 & 220 & 0.819 & -0.111 & 0.150 & 0.155 & -0.005 & I \\
\hline 116 & 2-hexanol & 4.202 & 0.254 & 1.780 & 280 & 0.819 & -0.111 & 0.141 & 0.143 & -0.002 & I \\
\hline 117 & 2-hexanol & 4.202 & 0.254 & 1.780 & 360 & 0.819 & -0.111 & 0.127 & 0.127 & 0.000 & I \\
\hline 118 & 2-hexanol & 4.202 & 0.254 & 1.780 & 370 & 0.819 & -0.111 & 0.125 & 0.125 & 0.000 & I \\
\hline 119 & 1-heptanol & 4.239 & 0.240 & 1.659 & 250 & 0.808 & -0.257 & 0.160 & 0.163 & -0.003 & / \\
\hline 120 & 1-heptanol & 4.239 & 0.240 & 1.659 & 260 & 0.808 & -0.257 & 0.159 & 0.161 & -0.002 & / \\
\hline 121 & 1-heptanol & 4.239 & 0.240 & 1.659 & 280 & 0.808 & -0.257 & 0.157 & 0.156 & 0.000 & I \\
\hline 122 & 1-heptanol & 4.239 & 0.240 & 1.659 & 300 & 0.808 & -0.257 & 0.153 & 0.152 & 0.001 & I \\
\hline 123 & 1-heptanol & 4.239 & 0.240 & 1.659 & 320 & 0.808 & -0.257 & 0.150 & 0.148 & 0.002 & / \\
\hline 124 & 1-heptanol & 4.239 & 0.240 & 1.659 & 360 & 0.808 & -0.257 & 0.142 & 0.140 & 0.002 & / \\
\hline 125 & 1-heptanol & 4.239 & 0.240 & 1.659 & 400 & 0.808 & -0.257 & 0.133 & 0.132 & 0.000 & I \\
\hline 126 & 1-heptanol & 4.239 & 0.240 & 1.659 & 500 & 0.808 & -0.257 & 0.111 & 0.112 & -0.001 & I \\
\hline 127 & 1-heptanol & 4.239 & 0.240 & 1.659 & 540 & 0.808 & -0.257 & 0.101 & 0.104 & -0.003 & 1 \\
\hline 128 & isoheptanol & 4.963 & 0.181 & 2.500 & 230 & 0.828 & -0.020 & 0.148 & 0.144 & 0.004 & 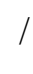 \\
\hline 129 & isoheptanol & 4.963 & 0.181 & 2.500 & 250 & 0.828 & -0.020 & 0.146 & 0.139 & 0.007 & 1 \\
\hline 130 & isoheptanol & 4.963 & 0.181 & 2.500 & 270 & 0.828 & -0.020 & 0.143 & 0.135 & 0.008 & I \\
\hline 131 & isoheptanol & 4.963 & 0.181 & 2.500 & 290 & 0.828 & -0.020 & 0.140 & 0.131 & 0.009 & I \\
\hline 132 & isoheptanol & 4.963 & 0.181 & 2.500 & 300 & 0.828 & -0.020 & 0.139 & 0.129 & 0.010 & I \\
\hline 133 & isoheptanol & 4.963 & 0.181 & 2.500 & 310 & 0.828 & -0.020 & 0.137 & 0.127 & 0.010 & I \\
\hline
\end{tabular}




\begin{tabular}{|c|c|c|c|c|c|c|c|c|c|c|c|}
\hline 134 & isoheptanol & 4.963 & 0.181 & 2.500 & 320 & 0.828 & -0.020 & 0.136 & 0.125 & 0.011 & 1 \\
\hline 135 & isoheptanol & 4.963 & 0.181 & 2.500 & 330 & 0.828 & -0.020 & 0.134 & 0.123 & 0.011 & I \\
\hline 136 & isoheptanol & 4.963 & 0.181 & 2.500 & 340 & 0.828 & -0.020 & 0.133 & 0.121 & 0.012 & 1 \\
\hline 137 & isoheptanol & 4.963 & 0.181 & 2.500 & 350 & 0.828 & -0.020 & 0.131 & 0.119 & 0.012 & / \\
\hline 138 & isoheptanol & 4.963 & 0.181 & 2.500 & 390 & 0.828 & -0.020 & 0.125 & 0.111 & 0.014 & / \\
\hline 139 & isoheptanol & 4.963 & 0.181 & 2.500 & 410 & 0.828 & -0.020 & 0.122 & 0.107 & 0.015 & / \\
\hline 140 & isoheptanol & 4.963 & 0.181 & 2.500 & 430 & 0.828 & -0.020 & 0.119 & 0.103 & 0.016 & / \\
\hline 141 & tert-heptanol & 4.290 & 0.240 & 1.862 & 320 & 0.871 & 0.025 & 0.136 & 0.124 & 0.012 & / \\
\hline 142 & tert-heptanol & 4.290 & 0.240 & 1.862 & 340 & 0.871 & 0.025 & 0.130 & 0.120 & 0.010 & l \\
\hline 143 & n-decyl alcohol & 4.408 & 0.211 & 1.522 & 323 & 0.902 & -0.255 & 0.159 & 0.157 & 0.002 & / \\
\hline 144 & n-decyl alcohol & 4.408 & 0.211 & 1.522 & 348 & 0.902 & -0.255 & 0.155 & 0.152 & 0.003 & / \\
\hline 145 & n-decyl alcohol & 4.408 & 0.211 & 1.522 & 398 & 0.902 & -0.255 & 0.147 & 0.142 & 0.005 & / \\
\hline 146 & n-decyl alcohol & 4.408 & 0.211 & 1.522 & 423 & 0.902 & -0.255 & 0.141 & 0.137 & 0.004 & l \\
\hline 147 & n-undecyl alcohol & 4.458 & 0.204 & 1.487 & 300 & 0.927 & -0.255 & 0.169 & 0.165 & 0.004 & l \\
\hline 148 & n-undecyl alcohol & 4.458 & 0.204 & 1.487 & 320 & 0.927 & -0.255 & 0.166 & 0.160 & 0.006 & / \\
\hline 149 & n-undecyl alcohol & 4.458 & 0.204 & 1.487 & 360 & 0.927 & -0.255 & 0.160 & 0.152 & 0.008 & / \\
\hline 150 & n-undecyl alcohol & 4.458 & 0.204 & 1.487 & 400 & 0.927 & -0.255 & 0.154 & 0.144 & 0.010 & l \\
\hline 151 & n-undecyl alcohol & 4.458 & 0.204 & 1.487 & 420 & 0.927 & -0.255 & 0.151 & 0.140 & 0.011 & / \\
\hline 152 & n-dodecyl alcohol & 4.506 & 0.198 & 1.457 & 310 & 0.951 & -0.255 & 0.166 & 0.165 & 0.001 & / \\
\hline 153 & n-dodecyl alcohol & 4.506 & 0.198 & 1.457 & 330 & 0.951 & -0.255 & 0.165 & 0.161 & 0.004 & l \\
\hline 154 & n-dodecyl alcohol & 4.506 & 0.198 & 1.457 & 350 & 0.951 & -0.255 & 0.162 & 0.157 & 0.005 & l \\
\hline 155 & n-dodecyl alcohol & 4.506 & 0.198 & 1.457 & 390 & 0.951 & -0.255 & 0.156 & 0.149 & 0.007 & / \\
\hline
\end{tabular}




\begin{tabular}{|c|c|c|c|c|c|c|c|c|c|c|c|}
\hline 156 & n-dodecyl alcohol & 4.506 & 0.198 & 1.457 & 430 & 0.951 & -0.255 & 0.149 & 0.141 & 0.008 & I \\
\hline 157 & n-dodecyl alcohol & 4.506 & 0.198 & 1.457 & 440 & 0.951 & -0.255 & 0.148 & 0.139 & 0.009 & I \\
\hline 158 & 1-tetradecanol & 4.596 & 0.189 & 1.405 & 340 & 0.993 & -0.254 & 0.166 & 0.165 & 0.001 & / \\
\hline 159 & 1-tetradecanol & 4.596 & 0.189 & 1.405 & 350 & 0.993 & -0.254 & 0.164 & 0.163 & 0.001 & / \\
\hline 160 & 1-tetradecanol & 4.596 & 0.189 & 1.405 & 360 & 0.993 & -0.254 & 0.163 & 0.161 & 0.002 & I \\
\hline 161 & 1-tetradecanol & 4.596 & 0.189 & 1.405 & 370 & 0.993 & -0.254 & 0.161 & 0.159 & 0.002 & / \\
\hline 162 & 1-tetradecanol & 4.596 & 0.189 & 1.405 & 390 & 0.993 & -0.254 & 0.158 & 0.155 & 0.003 & / \\
\hline 163 & 1-tetradecanol & 4.596 & 0.189 & 1.405 & 400 & 0.993 & -0.254 & 0.156 & 0.153 & 0.003 & t \\
\hline 164 & 1-tetradecanol & 4.596 & 0.189 & 1.405 & 430 & 0.993 & -0.254 & 0.151 & 0.147 & 0.004 & t \\
\hline 165 & 1-tetradecanol & 4.596 & 0.189 & 1.405 & 440 & 0.993 & -0.254 & 0.150 & 0.145 & 0.005 & 1 \\
\hline 166 & 1-tetradecanol & 4.596 & 0.189 & 1.405 & 450 & 0.993 & -0.254 & 0.148 & 0.143 & 0.005 & / \\
\hline 167 & n-octadecyl alcohol & 4.754 & 0.174 & 1.329 & 360 & 1.062 & -0.253 & 0.171 & 0.171 & 0.000 & I \\
\hline 168 & n-octadecyl alcohol & 4.754 & 0.174 & 1.329 & 390 & 1.062 & -0.253 & 0.165 & 0.165 & 0.000 & / \\
\hline 169 & n-octadecyl alcohol & 4.754 & 0.174 & 1.329 & 400 & 1.062 & -0.253 & 0.163 & 0.163 & 0.000 & 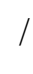 \\
\hline 170 & n-octadecyl alcohol & 4.754 & 0.174 & 1.329 & 420 & 1.062 & -0.253 & 0.160 & 0.159 & 0.001 & 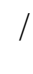 \\
\hline 171 & n-octadecyl alcohol & 4.754 & 0.174 & 1.329 & 440 & 1.062 & -0.253 & 0.156 & 0.155 & 0.001 & 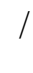 \\
\hline 172 & n-octyl alcohol & 4.299 & 0.228 & 1.607 & 280 & 0.843 & -0.256 & 0.160 & 0.159 & 0.001 & 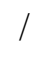 \\
\hline 173 & n-octyl alcohol & 4.299 & 0.228 & 1.607 & 320 & 0.843 & -0.256 & 0.153 & 0.151 & 0.002 & 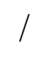 \\
\hline 174 & n-octyl alcohol & 4.299 & 0.228 & 1.607 & 400 & 0.843 & -0.256 & 0.137 & 0.135 & 0.002 & $t$ \\
\hline 175 & n-octyl alcohol & 4.299 & 0.228 & 1.607 & 440 & 0.843 & -0.256 & 0.128 & 0.127 & 0.001 & I \\
\hline 176 & n-octyl alcohol & 4.299 & 0.228 & 1.607 & 480 & 0.843 & -0.256 & 0.119 & 0.119 & 0.000 & 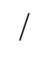 \\
\hline 177 & n-octyl alcohol & 4.299 & 0.228 & 1.607 & 500 & 0.843 & -0.256 & 0.114 & 0.115 & -0.001 & / \\
\hline
\end{tabular}




\begin{tabular}{|c|c|c|c|c|c|c|c|c|c|c|c|}
\hline 178 & n-octyl alcohol & 4.299 & 0.228 & 1.607 & 520 & 0.843 & -0.256 & 0.110 & 0.111 & -0.001 & I \\
\hline 179 & n-octyl alcohol & 4.299 & 0.228 & 1.607 & 560 & 0.843 & -0.256 & 0.100 & 0.103 & -0.003 & I \\
\hline 180 & n-octyl alcohol & 4.299 & 0.228 & 1.607 & 570 & 0.843 & -0.256 & 0.097 & 0.101 & -0.004 & / \\
\hline 181 & sec-octyl alcohol & 4.321 & 0.228 & 1.677 & 300 & 0.896 & -0.143 & 0.138 & 0.146 & -0.008 & / \\
\hline 182 & sec-octyl alcohol & 4.321 & 0.228 & 1.677 & 310 & 0.896 & -0.143 & 0.136 & 0.144 & -0.008 & / \\
\hline 183 & sec-octyl alcohol & 4.321 & 0.228 & 1.677 & 320 & 0.896 & -0.143 & 0.135 & 0.142 & -0.007 & I \\
\hline 184 & sec-octyl alcohol & 4.321 & 0.228 & 1.677 & 330 & 0.896 & -0.143 & 0.134 & 0.140 & -0.006 & l \\
\hline 185 & sec-octyl alcohol & 4.321 & 0.228 & 1.677 & 340 & 0.896 & -0.143 & 0.132 & 0.138 & -0.006 & / \\
\hline 186 & sec-octyl alcohol & 4.321 & 0.228 & 1.677 & 350 & 0.896 & -0.143 & 0.130 & 0.136 & -0.006 & l \\
\hline 187 & sec-octyl alcohol & 4.321 & 0.228 & 1.677 & 360 & 0.896 & -0.143 & 0.129 & 0.134 & -0.005 & I \\
\hline 188 & n-nonyl alcohol & 4.355 & 0.219 & 1.561 & 280 & 0.874 & -0.256 & 0.162 & 0.163 & -0.001 & I \\
\hline 189 & n-nonyl alcohol & 4.355 & 0.219 & 1.561 & 320 & 0.874 & -0.256 & 0.155 & 0.155 & 0.000 & t \\
\hline 190 & n-nonyl alcohol & 4.355 & 0.219 & 1.561 & 360 & 0.874 & -0.256 & 0.147 & 0.146 & 0.001 & / \\
\hline 191 & n-nonyl alcohol & 4.355 & 0.219 & 1.561 & 380 & 0.874 & -0.256 & 0.143 & 0.142 & 0.001 & / \\
\hline 192 & n-nonyl alcohol & 4.355 & 0.219 & 1.561 & 400 & 0.874 & -0.256 & 0.139 & 0.138 & 0.001 & / \\
\hline 193 & n-nonyl alcohol & 4.355 & 0.219 & 1.561 & 440 & 0.874 & -0.256 & 0.130 & 0.130 & 0.000 & / \\
\hline 194 & n-nonyl alcohol & 4.355 & 0.219 & 1.561 & 460 & 0.874 & -0.256 & 0.125 & 0.126 & -0.001 & 1 \\
\hline 195 & n-nonyl alcohol & 4.355 & 0.219 & 1.561 & 480 & 0.874 & -0.256 & 0.120 & 0.122 & -0.002 & / \\
\hline 196 & n-nonyl alcohol & 4.355 & 0.219 & 1.561 & 520 & 0.874 & -0.256 & 0.110 & 0.114 & -0.004 & / \\
\hline 197 & n-nonyl alcohol & 4.355 & 0.219 & 1.561 & 540 & 0.874 & -0.256 & 0.105 & 0.110 & -0.005 & 1 \\
\hline 198 & n-nonyl alcohol & 4.355 & 0.219 & 1.561 & 560 & 0.874 & -0.256 & 0.099 & 0.106 & -0.007 & / \\
\hline 199 & n-nonyl alcohol & 4.355 & 0.219 & 1.561 & 570 & 0.874 & -0.256 & 0.097 & 0.104 & -0.007 & l \\
\hline
\end{tabular}




\begin{tabular}{|c|c|c|c|c|c|c|c|c|c|c|c|}
\hline 200 & diethyl ether & 3.544 & 0.297 & 1.426 & 230 & 0.888 & -0.153 & 0.157 & 0.139 & 0.018 & I \\
\hline 201 & diethyl ether & 3.544 & 0.297 & 1.426 & 240 & 0.888 & -0.153 & 0.153 & 0.137 & 0.016 & / \\
\hline 202 & diethyl ether & 3.544 & 0.297 & 1.426 & 250 & 0.888 & -0.153 & 0.149 & 0.135 & 0.014 & I \\
\hline 203 & diethyl ether & 3.544 & 0.297 & 1.426 & 260 & 0.888 & -0.153 & 0.146 & 0.133 & 0.013 & I \\
\hline 204 & diethyl ether & 3.544 & 0.297 & 1.426 & 300 & 0.888 & -0.153 & 0.130 & 0.125 & 0.005 & / \\
\hline 205 & diisopropyl ether & 3.839 & 0.254 & 1.378 & 280 & 1.036 & 0.109 & 0.114 & 0.122 & -0.008 & I \\
\hline 206 & diisopropyl ether & 3.839 & 0.254 & 1.378 & 300 & 1.036 & 0.109 & 0.111 & 0.118 & -0.007 & I \\
\hline 207 & dipropyl ether & 3.763 & 0.254 & 1.568 & 280 & 1.030 & -0.184 & 0.136 & 0.124 & 0.012 & / \\
\hline 208 & dipropyl ether & 3.763 & 0.254 & 1.568 & 300 & 1.030 & -0.184 & 0.131 & 0.120 & 0.011 & I \\
\hline 209 & ethyl-n-butyl ether & 3.763 & 0.254 & 1.568 & 320 & 1.019 & -0.184 & 0.122 & 0.116 & 0.006 & I \\
\hline 210 & ethyl-n-butyl ether & 3.763 & 0.254 & 1.568 & 330 & 1.019 & -0.184 & 0.118 & 0.114 & 0.004 & I \\
\hline 211 & ethyl-n-butyl ether & 3.763 & 0.254 & 1.568 & 340 & 1.019 & -0.184 & 0.114 & 0.112 & 0.002 & I \\
\hline 212 & diamyl ether & 4.096 & 0.211 & 1.480 & 293 & 1.248 & -0.209 & 0.132 & 0.136 & -0.004 & / \\
\hline 213 & diamyl ether & 4.096 & 0.211 & 1.480 & 313 & 1.248 & -0.209 & 0.127 & 0.132 & -0.005 & I \\
\hline 214 & diamyl ether & 4.096 & 0.211 & 1.480 & 333 & 1.248 & -0.209 & 0.123 & 0.128 & -0.005 & t \\
\hline 215 & diamyl ether & 4.096 & 0.211 & 1.480 & 353 & 1.248 & -0.209 & 0.120 & 0.124 & -0.004 & / \\
\hline 216 & diamyl ether & 4.096 & 0.211 & 1.480 & 373 & 1.248 & -0.209 & 0.116 & 0.120 & -0.004 & 1 \\
\hline 217 & diamyl ether & 4.096 & 0.211 & 1.480 & 393 & 1.248 & -0.209 & 0.112 & 0.116 & -0.004 & I \\
\hline 218 & diamyl ether & 4.096 & 0.211 & 1.480 & 413 & 1.248 & -0.209 & 0.109 & 0.112 & -0.003 & / \\
\hline 219 & ethyl-octyl ether & 4.096 & 0.211 & 1.480 & 300 & 1.179 & -0.209 & 0.130 & 0.135 & -0.005 & 1 \\
\hline 220 & ethyl-octyl ether & 4.096 & 0.211 & 1.480 & 320 & 1.179 & -0.209 & 0.126 & 0.131 & -0.005 & l \\
\hline 221 & ethyl-octyl ether & 4.096 & 0.211 & 1.480 & 340 & 1.179 & -0.209 & 0.122 & 0.127 & -0.005 & I \\
\hline
\end{tabular}




\begin{tabular}{|c|c|c|c|c|c|c|c|c|c|c|c|}
\hline 222 & di-n-hexyl ether & 4.228 & 0.198 & 1.434 & 440 & 1.331 & -0.216 & 0.109 & 0.113 & -0.004 & I \\
\hline 223 & di-n-heptyl ether & 4.345 & 0.189 & 1.393 & 300 & 1.404 & -0.221 & 0.137 & 0.149 & -0.012 & / \\
\hline 224 & di-n-heptyl ether & 4.345 & 0.189 & 1.393 & 360 & 1.404 & -0.221 & 0.127 & 0.137 & -0.010 & I \\
\hline 225 & di-n-heptyl ether & 4.345 & 0.189 & 1.393 & 380 & 1.404 & -0.221 & 0.124 & 0.133 & -0.009 & I \\
\hline 226 & di-n-heptyl ether & 4.345 & 0.189 & 1.393 & 400 & 1.404 & -0.221 & 0.120 & 0.129 & -0.009 & I \\
\hline 227 & di-n-heptyl ether & 4.345 & 0.189 & 1.393 & 420 & 1.404 & -0.221 & 0.117 & 0.124 & -0.007 & 1 \\
\hline 228 & di-n-heptyl ether & 4.345 & 0.189 & 1.393 & 440 & 1.404 & -0.221 & 0.113 & 0.120 & -0.007 & / \\
\hline 229 & di-n-heptyl ether & 4.345 & 0.189 & 1.393 & 460 & 1.404 & -0.221 & 0.110 & 0.116 & -0.006 & t \\
\hline 230 & di-n-heptyl ether & 4.345 & 0.189 & 1.393 & 480 & 1.404 & -0.221 & 0.106 & 0.112 & -0.006 & t \\
\hline 231 & octyl ether & 4.450 & 0.181 & 1.359 & 300 & 1.469 & -0.224 & 0.142 & 0.155 & -0.013 & 1 \\
\hline 232 & octyl ether & 4.450 & 0.181 & 1.359 & 360 & 1.469 & -0.224 & 0.131 & 0.143 & -0.012 & I \\
\hline 233 & octyl ether & 4.450 & 0.181 & 1.359 & 380 & 1.469 & -0.224 & 0.127 & 0.139 & -0.012 & I \\
\hline 234 & octyl ether & 4.450 & 0.181 & 1.359 & 440 & 1.469 & -0.224 & 0.117 & 0.127 & -0.010 & / \\
\hline 235 & octyl ether & 4.450 & 0.181 & 1.359 & 460 & 1.469 & -0.224 & 0.113 & 0.123 & -0.010 & / \\
\hline 236 & octyl ether & 4.450 & 0.181 & 1.359 & 480 & 1.469 & -0.224 & 0.110 & 0.119 & -0.009 & I \\
\hline 237 & dibutyl ether & 3.943 & 0.228 & 1.530 & 280 & 1.149 & -0.200 & 0.137 & 0.131 & 0.006 & t \\
\hline 238 & dibutyl ether & 3.943 & 0.228 & 1.530 & 320 & 1.149 & -0.200 & 0.126 & 0.123 & 0.003 & 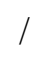 \\
\hline 239 & dibutyl ether & 3.943 & 0.228 & 1.530 & 340 & 1.149 & -0.200 & 0.120 & 0.119 & 0.001 & 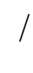 \\
\hline 240 & dibutyl ether & 3.943 & 0.228 & 1.530 & 360 & 1.149 & -0.200 & 0.115 & 0.115 & 0.000 & I \\
\hline 241 & dibutyl ether & 3.943 & 0.228 & 1.530 & 380 & 1.149 & -0.200 & 0.110 & 0.111 & -0.001 & I \\
\hline 242 & Ethyl hexyl ether & 3.931 & 0.242 & 1.619 & 280 & 0.945 & -0.217 & 0.130 & 0.133 & -0.003 & 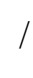 \\
\hline 243 & Ethyl hexyl ether & 3.931 & 0.242 & 1.619 & 300 & 0.945 & -0.217 & 0.126 & 0.129 & -0.003 & / \\
\hline
\end{tabular}




\begin{tabular}{|c|c|c|c|c|c|c|c|c|c|c|c|}
\hline 244 & Ethyl hexyl ether & 3.931 & 0.242 & 1.619 & 340 & 0.945 & -0.217 & 0.117 & 0.121 & -0.004 & 1 \\
\hline 245 & Ethyl hexyl ether & 3.931 & 0.242 & 1.619 & 380 & 0.945 & -0.217 & 0.108 & 0.113 & -0.005 & / \\
\hline 246 & Ethyl hexyl ether & 3.931 & 0.242 & 1.619 & 390 & 0.945 & -0.217 & 0.106 & 0.111 & -0.005 & / \\
\hline 247 & Isobutyric aldehyde & 4.320 & 0.335 & 2.035 & 280 & 1.573 & -0.034 & 0.148 & 0.148 & 0.000 & / \\
\hline 248 & Isobutyric aldehyde & 4.320 & 0.335 & 2.035 & 290 & 1.573 & -0.034 & 0.147 & 0.146 & 0.001 & / \\
\hline 249 & Isobutyric aldehyde & 4.320 & 0.335 & 2.035 & 300 & 1.573 & -0.034 & 0.146 & 0.144 & 0.002 & / \\
\hline 250 & Isobutyric aldehyde & 4.320 & 0.335 & 2.035 & 310 & 1.573 & -0.034 & 0.144 & 0.142 & 0.002 & \\
\hline 251 & Isobutyric aldehyde & 4.320 & 0.335 & 2.035 & 320 & 1.573 & -0.034 & 0.142 & 0.140 & 0.002 & t \\
\hline 252 & Isobutyric aldehyde & 4.320 & 0.335 & 2.035 & 330 & 1.573 & -0.034 & 0.140 & 0.138 & 0.002 & \\
\hline 253 & Isobutyric aldehyde & 4.320 & 0.335 & 2.035 & 340 & 1.573 & -0.034 & 0.140 & 0.136 & 0.004 & , \\
\hline 254 & Isobutyric aldehyde & 4.320 & 0.335 & 2.035 & 350 & 1.573 & -0.034 & 0.138 & 0.134 & 0.004 & 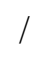 \\
\hline 255 & Isobutyric aldehyde & 4.320 & 0.335 & 2.035 & 370 & 1.573 & -0.034 & 0.135 & 0.130 & 0.005 & 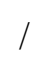 \\
\hline 256 & Isobutyric aldehyde & 4.320 & 0.335 & 2.035 & 380 & 1.573 & -0.034 & 0.134 & 0.128 & 0.006 & 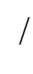 \\
\hline 257 & Isobutyric aldehyde & 4.320 & 0.335 & 2.035 & 390 & 1.573 & -0.034 & 0.132 & 0.126 & 0.006 & t \\
\hline 258 & Isobutyric aldehyde & 4.320 & 0.335 & 2.035 & 410 & 1.573 & -0.034 & 0.129 & 0.122 & 0.007 & \\
\hline 259 & Isobutyric aldehyde & 4.320 & 0.335 & 2.035 & 420 & 1.573 & -0.034 & 0.128 & 0.120 & 0.008 & \\
\hline 260 & Isobutyric aldehyde & 4.320 & 0.335 & 2.035 & 430 & 1.573 & -0.034 & 0.126 & 0.118 & 0.008 & \\
\hline 261 & valeraldehyde & 4.354 & 0.300 & 2.092 & 280 & 1.571 & -0.219 & 0.144 & 0.148 & -0.004 & \\
\hline 262 & valeraldehyde & 4.354 & 0.300 & 2.092 & 290 & 1.571 & -0.219 & 0.142 & 0.146 & -0.004 & t \\
\hline 263 & valeraldehyde & 4.354 & 0.300 & 2.092 & 303 & 1.571 & -0.219 & 0.139 & 0.144 & -0.005 & I \\
\hline 264 & valeraldehyde & 4.354 & 0.300 & 2.092 & 310 & 1.571 & -0.219 & 0.137 & 0.142 & -0.005 & I \\
\hline 265 & valeraldehyde & 4.354 & 0.300 & 2.092 & 320 & 1.571 & -0.219 & 0.134 & 0.140 & -0.006 & l \\
\hline
\end{tabular}




\begin{tabular}{|c|c|c|c|c|c|c|c|c|c|c|c|}
\hline 266 & valeraldehyde & 4.354 & 0.300 & 2.092 & 330 & 1.571 & -0.219 & 0.132 & 0.138 & -0.006 & I \\
\hline 267 & valeraldehyde & 4.354 & 0.300 & 2.092 & 350 & 1.571 & -0.219 & 0.126 & 0.134 & -0.008 & / \\
\hline 268 & valeraldehyde & 4.354 & 0.300 & 2.092 & 360 & 1.571 & -0.219 & 0.124 & 0.132 & -0.008 & / \\
\hline 269 & valeraldehyde & 4.354 & 0.300 & 2.092 & 370 & 1.571 & -0.219 & 0.122 & 0.130 & -0.008 & / \\
\hline 270 & isovaleraldehyde & 4.375 & 0.300 & 2.092 & 290 & 1.653 & -0.082 & 0.134 & 0.139 & -0.005 & I \\
\hline 271 & isovaleraldehyde & 4.375 & 0.300 & 2.092 & 300 & 1.653 & -0.082 & 0.132 & 0.137 & -0.005 & / \\
\hline 272 & isovaleraldehyde & 4.375 & 0.300 & 2.092 & 301 & 1.653 & -0.082 & 0.131 & 0.137 & -0.006 & I \\
\hline 273 & isovaleraldehyde & 4.375 & 0.300 & 2.092 & 310 & 1.653 & -0.082 & 0.129 & 0.135 & -0.006 & 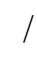 \\
\hline 274 & isovaleraldehyde & 4.375 & 0.300 & 2.092 & 320 & 1.653 & -0.082 & 0.126 & 0.133 & -0.007 & / \\
\hline 275 & isovaleraldehyde & 4.375 & 0.300 & 2.092 & 330 & 1.653 & -0.082 & 0.124 & 0.131 & -0.007 & I \\
\hline 276 & isovaleraldehyde & 4.375 & 0.300 & 2.092 & 335 & 1.653 & -0.082 & 0.122 & 0.130 & -0.008 & / \\
\hline 277 & isovaleraldehyde & 4.375 & 0.300 & 2.092 & 340 & 1.653 & -0.082 & 0.121 & 0.129 & -0.008 & / \\
\hline 278 & isovaleraldehyde & 4.375 & 0.300 & 2.092 & 350 & 1.653 & -0.082 & 0.119 & 0.127 & -0.008 & / \\
\hline 279 & isovaleraldehyde & 4.375 & 0.300 & 2.092 & 360 & 1.653 & -0.082 & 0.116 & 0.125 & -0.009 & l \\
\hline 280 & isovaleraldehyde & 4.375 & 0.300 & 2.092 & 362 & 1.653 & -0.082 & 0.115 & 0.124 & -0.009 & / \\
\hline 281 & decyl aldehyde & 4.595 & 0.221 & 1.717 & 260 & 1.971 & -0.241 & 0.152 & 0.162 & -0.010 & t \\
\hline 282 & decyl aldehyde & 4.595 & 0.221 & 1.717 & 270 & 1.971 & -0.241 & 0.150 & 0.160 & -0.010 & 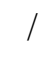 \\
\hline 283 & decyl aldehyde & 4.595 & 0.221 & 1.717 & 290 & 1.971 & -0.241 & 0.146 & 0.155 & -0.009 & 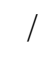 \\
\hline 284 & decyl aldehyde & 4.595 & 0.221 & 1.717 & 310 & 1.971 & -0.241 & 0.142 & 0.151 & -0.009 & t \\
\hline 285 & decyl aldehyde & 4.595 & 0.221 & 1.717 & 320 & 1.971 & -0.241 & 0.140 & 0.149 & -0.009 & I \\
\hline 286 & decyl aldehyde & 4.595 & 0.221 & 1.717 & 340 & 1.971 & -0.241 & 0.136 & 0.145 & -0.009 & I \\
\hline 287 & decyl aldehyde & 4.595 & 0.221 & 1.717 & 350 & 1.971 & -0.241 & 0.134 & 0.143 & -0.009 & / \\
\hline
\end{tabular}




\begin{tabular}{|c|c|c|c|c|c|c|c|c|c|c|c|}
\hline 288 & decyl aldehyde & 4.595 & 0.221 & 1.717 & 370 & 1.971 & -0.241 & 0.130 & 0.139 & -0.009 & I \\
\hline 289 & decyl aldehyde & 4.595 & 0.221 & 1.717 & 380 & 1.971 & -0.241 & 0.128 & 0.137 & -0.009 & I \\
\hline 290 & decyl aldehyde & 4.595 & 0.221 & 1.717 & 400 & 1.971 & -0.241 & 0.124 & 0.133 & -0.009 & I \\
\hline 291 & lauric aldehyde & 4.678 & 0.206 & 1.626 & 330 & 2.080 & -0.243 & 0.146 & 0.152 & -0.006 & I \\
\hline 292 & lauric aldehyde & 4.678 & 0.206 & 1.626 & 370 & 2.080 & -0.243 & 0.139 & 0.144 & -0.005 & / \\
\hline 293 & lauric aldehyde & 4.678 & 0.206 & 1.626 & 390 & 2.080 & -0.243 & 0.135 & 0.140 & -0.005 & I \\
\hline 294 & lauric aldehyde & 4.678 & 0.206 & 1.626 & 430 & 2.080 & -0.243 & 0.128 & 0.132 & -0.004 & I \\
\hline 295 & lauric aldehyde & 4.678 & 0.206 & 1.626 & 450 & 2.080 & -0.243 & 0.124 & 0.128 & -0.004 & I \\
\hline 296 & Acetaldehyde & 4.174 & 0.491 & 2.128 & 288 & 1.131 & -0.094 & 0.185 & 0.187 & -0.002 & I \\
\hline 297 & propionaldehyde & 4.238 & 0.390 & 2.161 & 273 & 1.304 & -0.174 & 0.168 & 0.165 & 0.002 & I \\
\hline 298 & propionaldehyde & 4.238 & 0.390 & 2.161 & 288 & 1.304 & -0.174 & 0.163 & 0.162 & 0.001 & I \\
\hline 299 & propionaldehyde & 4.238 & 0.390 & 2.161 & 298 & 1.304 & -0.174 & 0.160 & 0.160 & 0.000 & I \\
\hline 300 & butyraldehyde & 4.297 & 0.335 & 2.189 & 273 & 1.451 & -0.204 & 0.151 & 0.152 & -0.001 & / \\
\hline 301 & butyraldehyde & 4.297 & 0.335 & 2.189 & 288 & 1.451 & -0.204 & 0.148 & 0.149 & -0.001 & I \\
\hline 302 & butyraldehyde & 4.297 & 0.335 & 2.189 & 298 & 1.451 & -0.204 & 0.145 & 0.147 & -0.002 & I \\
\hline 303 & butyraldehyde & 4.297 & 0.335 & 2.189 & 323 & 1.451 & -0.204 & 0.139 & 0.142 & -0.003 & , \\
\hline 304 & hexanal & 4.407 & 0.275 & 1.995 & 273 & 1.673 & -0.227 & 0.143 & 0.150 & -0.007 & 1 \\
\hline 305 & hexanal & 4.407 & 0.275 & 1.995 & 288 & 1.673 & -0.227 & 0.139 & 0.147 & -0.008 & I \\
\hline 306 & hexanal & 4.407 & 0.275 & 1.995 & 298 & 1.673 & -0.227 & 0.137 & 0.145 & -0.009 & / \\
\hline 307 & hexanal & 4.407 & 0.275 & 1.995 & 323 & 1.673 & -0.227 & 0.131 & 0.140 & -0.010 & 1 \\
\hline 308 & heptanal & 4.457 & 0.256 & 1.909 & 288 & 1.762 & -0.233 & 0.140 & 0.149 & -0.009 & l \\
\hline 309 & heptanal & 4.457 & 0.256 & 1.909 & 323 & 1.762 & -0.233 & 0.132 & 0.142 & -0.010 & I \\
\hline
\end{tabular}




\begin{tabular}{|c|c|c|c|c|c|c|c|c|c|c|c|}
\hline 310 & octanal & 4.505 & 0.242 & 1.835 & 298 & 1.839 & -0.236 & 0.139 & 0.149 & -0.011 & I \\
\hline 311 & octanal & 4.505 & 0.242 & 1.835 & 323 & 1.839 & -0.236 & 0.133 & 0.144 & -0.011 & 1 \\
\hline 312 & nonanal & 4.551 & 0.230 & 1.772 & 273 & 1.908 & -0.239 & 0.146 & 0.156 & -0.010 & I \\
\hline 313 & nonanal & 4.551 & 0.230 & 1.772 & 288 & 1.908 & -0.239 & 0.143 & 0.153 & -0.011 & I \\
\hline 314 & nonanal & 4.551 & 0.230 & 1.772 & 323 & 1.908 & -0.239 & 0.135 & 0.146 & -0.011 & I \\
\hline 315 & 2-methyl hexaldehyde & 4.477 & 0.256 & 1.966 & 288 & 1.942 & -0.133 & 0.139 & 0.139 & 0.000 & 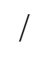 \\
\hline 316 & 2-methyl hexaldehyde & 4.477 & 0.256 & 1.966 & 298 & 1.942 & -0.133 & 0.136 & 0.137 & -0.001 & I \\
\hline 317 & 2-methyl hexaldehyde & 4.477 & 0.256 & 1.966 & 323 & 1.942 & -0.133 & 0.130 & 0.132 & -0.002 & t \\
\hline 318 & 3-methyl hexaldehyde & 4.477 & 0.256 & 2.091 & 288 & 1.876 & -0.133 & 0.144 & 0.134 & 0.010 & t \\
\hline 319 & 3-methyl hexaldehyde & 4.477 & 0.256 & 2.091 & 298 & 1.876 & -0.133 & 0.141 & 0.132 & 0.010 & I \\
\hline 320 & 3-methyl hexaldehyde & 4.477 & 0.256 & 2.091 & 323 & 1.876 & -0.133 & 0.135 & 0.127 & 0.008 & I \\
\hline 321 & 2-ethylhexaldehyde & 4.524 & 0.242 & 1.900 & 273 & 2.089 & -0.149 & 0.131 & 0.144 & -0.013 & I \\
\hline 322 & 2-ethylhexaldehyde & 4.524 & 0.242 & 1.900 & 288 & 2.089 & -0.149 & 0.128 & 0.141 & -0.013 & / \\
\hline 323 & 2-ethylhexaldehyde & 4.524 & 0.242 & 1.900 & 298 & 2.089 & -0.149 & 0.125 & 0.139 & -0.014 & I \\
\hline 324 & 2-ethylhexaldehyde & 4.524 & 0.242 & 1.900 & 323 & 2.089 & -0.149 & 0.120 & 0.134 & -0.014 & / \\
\hline 325 & Formic acid & 4.575 & 0.655 & 2.322 & 300 & 1.357 & -0.470 & 0.267 & 0.271 & -0.004 & 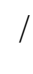 \\
\hline 326 & Formic acid & 4.575 & 0.655 & 2.322 & 310 & 1.357 & -0.470 & 0.266 & 0.269 & -0.003 & 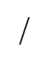 \\
\hline 327 & Formic acid & 4.575 & 0.655 & 2.322 & 320 & 1.357 & -0.470 & 0.265 & 0.267 & -0.002 & 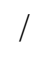 \\
\hline 328 & Formic acid & 4.575 & 0.655 & 2.322 & 340 & 1.357 & -0.470 & 0.264 & 0.263 & 0.001 & 1 \\
\hline 329 & Formic acid & 4.575 & 0.655 & 2.322 & 350 & 1.357 & -0.470 & 0.263 & 0.261 & 0.002 & I \\
\hline 330 & Formic acid & 4.575 & 0.655 & 2.322 & 360 & 1.357 & -0.470 & 0.262 & 0.259 & 0.003 & 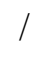 \\
\hline 331 & Formic acid & 4.575 & 0.655 & 2.322 & 370 & 1.357 & -0.470 & 0.261 & 0.257 & 0.004 & I \\
\hline
\end{tabular}




\begin{tabular}{|c|c|c|c|c|c|c|c|c|c|c|c|}
\hline 332 & Acetic acid & 4.627 & 0.500 & 2.406 & 290 & 1.803 & 0.358 & 0.165 & 0.172 & -0.007 & I \\
\hline 333 & Acetic acid & 4.627 & 0.500 & 2.406 & 300 & 1.803 & 0.358 & 0.164 & 0.170 & -0.006 & I \\
\hline 334 & Acetic acid & 4.627 & 0.500 & 2.406 & 330 & 1.803 & 0.358 & 0.159 & 0.164 & -0.005 & I \\
\hline 335 & Acetic acid & 4.627 & 0.500 & 2.406 & 340 & 1.803 & 0.358 & 0.158 & 0.162 & -0.004 & I \\
\hline 336 & Acetic acid & 4.627 & 0.500 & 2.406 & 350 & 1.803 & 0.358 & 0.156 & 0.160 & -0.004 & I \\
\hline 337 & Acetic acid & 4.627 & 0.500 & 2.406 & 360 & 1.803 & 0.358 & 0.156 & 0.158 & -0.002 & I \\
\hline 338 & Propionic acid & 4.668 & 0.415 & 2.404 & 280 & 2.021 & 0.154 & 0.146 & 0.161 & -0.015 & I \\
\hline 339 & Propionic acid & 4.668 & 0.415 & 2.404 & 300 & 2.021 & 0.154 & 0.144 & 0.157 & -0.013 & t \\
\hline 340 & Propionic acid & 4.668 & 0.415 & 2.404 & 330 & 2.021 & 0.154 & 0.141 & 0.151 & -0.010 & / \\
\hline 341 & Propionic acid & 4.668 & 0.415 & 2.404 & 340 & 2.021 & 0.154 & 0.140 & 0.149 & -0.009 & I \\
\hline 342 & Propionic acid & 4.668 & 0.415 & 2.404 & 350 & 2.021 & 0.154 & 0.138 & 0.147 & -0.009 & I \\
\hline 343 & Propionic acid & 4.668 & 0.415 & 2.404 & 380 & 2.021 & 0.154 & 0.135 & 0.141 & -0.006 & I \\
\hline 344 & Propionic acid & 4.668 & 0.415 & 2.404 & 400 & 2.021 & 0.154 & 0.133 & 0.137 & -0.004 & / \\
\hline 345 & valeric acid & 4.745 & 0.326 & 2.292 & 290 & 2.353 & -0.002 & 0.140 & 0.149 & -0.009 & I \\
\hline 346 & valeric acid & 4.745 & 0.326 & 2.292 & 300 & 2.353 & -0.002 & 0.139 & 0.147 & -0.008 & I \\
\hline 347 & valeric acid & 4.745 & 0.326 & 2.292 & 310 & 2.353 & -0.002 & 0.138 & 0.145 & -0.007 & t \\
\hline 348 & valeric acid & 4.745 & 0.326 & 2.292 & 320 & 2.353 & -0.002 & 0.137 & 0.143 & -0.006 & 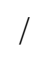 \\
\hline 349 & valeric acid & 4.745 & 0.326 & 2.292 & 330 & 2.353 & -0.002 & 0.135 & 0.141 & -0.006 & 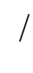 \\
\hline 350 & valeric acid & 4.745 & 0.326 & 2.292 & 340 & 2.353 & -0.002 & 0.134 & 0.139 & -0.005 & $t$ \\
\hline 351 & valeric acid & 4.745 & 0.326 & 2.292 & 350 & 2.353 & -0.002 & 0.133 & 0.137 & -0.004 & I \\
\hline 352 & valeric acid & 4.745 & 0.326 & 2.292 & 370 & 2.353 & -0.002 & 0.130 & 0.133 & -0.003 & , \\
\hline 353 & valeric acid & 4.745 & 0.326 & 2.292 & 380 & 2.353 & -0.002 & 0.129 & 0.131 & -0.002 & I \\
\hline
\end{tabular}




\begin{tabular}{|c|c|c|c|c|c|c|c|c|c|c|c|}
\hline 354 & valeric acid & 4.745 & 0.326 & 2.292 & 400 & 2.353 & -0.002 & 0.127 & 0.127 & 0.000 & I \\
\hline 355 & valeric acid & 4.745 & 0.326 & 2.292 & 410 & 2.353 & -0.002 & 0.125 & 0.125 & 0.000 & / \\
\hline 356 & valeric acid & 4.745 & 0.326 & 2.292 & 420 & 2.353 & -0.002 & 0.124 & 0.123 & 0.001 & I \\
\hline 357 & valeric acid & 4.745 & 0.326 & 2.292 & 430 & 2.353 & -0.002 & 0.123 & 0.121 & 0.002 & I \\
\hline 358 & caproic acid & 4.781 & 0.300 & 2.181 & 290 & 2.481 & -0.041 & 0.143 & 0.150 & -0.007 & / \\
\hline 359 & caproic acid & 4.781 & 0.300 & 2.181 & 300 & 2.481 & -0.041 & 0.141 & 0.148 & -0.007 & I \\
\hline 360 & caproic acid & 4.781 & 0.300 & 2.181 & 340 & 2.481 & -0.041 & 0.132 & 0.140 & -0.008 & I \\
\hline 361 & caproic acid & 4.781 & 0.300 & 2.181 & 350 & 2.481 & -0.041 & 0.130 & 0.138 & -0.008 & I \\
\hline 362 & enanthic acid & 4.816 & 0.280 & 2.084 & 280 & 2.592 & -0.069 & 0.143 & 0.154 & -0.011 & / \\
\hline 363 & enanthic acid & 4.816 & 0.280 & 2.084 & 290 & 2.592 & -0.069 & 0.142 & 0.152 & -0.010 & / \\
\hline 364 & enanthic acid & 4.816 & 0.280 & 2.084 & 310 & 2.592 & -0.069 & 0.139 & 0.148 & -0.009 & I \\
\hline 365 & enanthic acid & 4.816 & 0.280 & 2.084 & 320 & 2.592 & -0.069 & 0.137 & 0.146 & -0.009 & I \\
\hline 366 & enanthic acid & 4.816 & 0.280 & 2.084 & 330 & 2.592 & -0.069 & 0.136 & 0.144 & -0.008 & I \\
\hline 367 & enanthic acid & 4.816 & 0.280 & 2.084 & 350 & 2.592 & -0.069 & 0.133 & 0.140 & -0.007 & / \\
\hline 368 & capric acid & 4.914 & 0.240 & 1.864 & 310 & 2.856 & -0.120 & 0.150 & 0.154 & -0.004 & / \\
\hline 369 & capric acid & 4.914 & 0.240 & 1.864 & 330 & 2.856 & -0.120 & 0.147 & 0.150 & -0.003 & I \\
\hline 370 & capric acid & 4.914 & 0.240 & 1.864 & 350 & 2.856 & -0.120 & 0.144 & 0.146 & -0.002 & / \\
\hline 371 & capric acid & 4.914 & 0.240 & 1.864 & 370 & 2.856 & -0.120 & 0.141 & 0.142 & -0.001 & I \\
\hline 372 & capric acid & 4.914 & 0.240 & 1.864 & 390 & 2.856 & -0.120 & 0.137 & 0.138 & -0.001 & I \\
\hline 373 & capric acid & 4.914 & 0.240 & 1.864 & 410 & 2.856 & -0.120 & 0.134 & 0.134 & 0.000 & I \\
\hline 374 & capric acid & 4.914 & 0.240 & 1.864 & 430 & 2.856 & -0.120 & 0.131 & 0.130 & 0.001 & 1 \\
\hline 375 & capric acid & 4.914 & 0.240 & 1.864 & 460 & 2.856 & -0.120 & 0.126 & 0.124 & 0.002 & l \\
\hline
\end{tabular}




\begin{tabular}{|c|c|c|c|c|c|c|c|c|c|c|c|}
\hline 376 & Undecanoic acid & 4.945 & 0.230 & 1.808 & 330 & 2.928 & -0.131 & 0.152 & 0.152 & 0.000 & I \\
\hline 377 & Undecanoic acid & 4.945 & 0.230 & 1.808 & 340 & 2.928 & -0.131 & 0.150 & 0.150 & 0.000 & I \\
\hline 378 & Undecanoic acid & 4.945 & 0.230 & 1.808 & 350 & 2.928 & -0.131 & 0.149 & 0.148 & 0.001 & I \\
\hline 379 & lauric acid & 4.975 & 0.222 & 1.759 & 330 & 2.994 & -0.141 & 0.159 & 0.154 & 0.005 & I \\
\hline 380 & myristic acid & 5.032 & 0.209 & 1.677 & 330 & 3.112 & -0.155 & 0.160 & 0.157 & 0.003 & / \\
\hline 381 & myristic acid & 5.032 & 0.209 & 1.677 & 340 & 3.112 & -0.155 & 0.158 & 0.155 & 0.003 & / \\
\hline 382 & myristic acid & 5.032 & 0.209 & 1.677 & 350 & 3.112 & -0.155 & 0.156 & 0.153 & 0.003 & I \\
\hline 383 & myristic acid & 5.032 & 0.209 & 1.677 & 370 & 3.112 & -0.155 & 0.152 & 0.149 & 0.003 & t \\
\hline 384 & myristic acid & 5.032 & 0.209 & 1.677 & 390 & 3.112 & -0.155 & 0.148 & 0.145 & 0.003 & / \\
\hline 385 & myristic acid & 5.032 & 0.209 & 1.677 & 400 & 3.112 & -0.155 & 0.146 & 0.143 & 0.003 & I \\
\hline 386 & myristic acid & 5.032 & 0.209 & 1.677 & 410 & 3.112 & -0.155 & 0.143 & 0.141 & 0.002 & / \\
\hline 387 & myristic acid & 5.032 & 0.209 & 1.677 & 430 & 3.112 & -0.155 & 0.140 & 0.137 & 0.003 & / \\
\hline 388 & myristic acid & 5.032 & 0.209 & 1.677 & 460 & 3.112 & -0.155 & 0.134 & 0.131 & 0.003 & / \\
\hline 389 & palmitic acid & 5.086 & 0.199 & 1.610 & 350 & 3.215 & -0.167 & 0.164 & 0.157 & 0.007 & l \\
\hline 390 & palmitic acid & 5.086 & 0.199 & 1.610 & 360 & 3.215 & -0.167 & 0.162 & 0.155 & 0.007 & / \\
\hline 391 & palmitic acid & 5.086 & 0.199 & 1.610 & 370 & 3.215 & -0.167 & 0.160 & 0.153 & 0.007 & t \\
\hline 392 & palmitic acid & 5.086 & 0.199 & 1.610 & 400 & 3.215 & -0.167 & 0.152 & 0.147 & 0.005 & 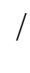 \\
\hline 393 & palmitic acid & 5.086 & 0.199 & 1.610 & 410 & 3.215 & -0.167 & 0.150 & 0.145 & 0.005 & t \\
\hline 394 & palmitic acid & 5.086 & 0.199 & 1.610 & 420 & 3.215 & -0.167 & 0.148 & 0.143 & 0.005 & l \\
\hline 395 & palmitic acid & 5.086 & 0.199 & 1.610 & 430 & 3.215 & -0.167 & 0.146 & 0.141 & 0.005 & I \\
\hline 396 & palmitic acid & 5.086 & 0.199 & 1.610 & 440 & 3.215 & -0.167 & 0.144 & 0.139 & 0.005 & t \\
\hline 397 & palmitic acid & 5.086 & 0.199 & 1.610 & 450 & 3.215 & -0.167 & 0.142 & 0.137 & 0.005 & l \\
\hline
\end{tabular}




\begin{tabular}{|c|c|c|c|c|c|c|c|c|c|c|c|}
\hline 398 & palmitic acid & 5.086 & 0.199 & 1.610 & 460 & 3.215 & -0.167 & 0.140 & 0.135 & 0.005 & I \\
\hline 399 & heptadecanoic acid & 5.112 & 0.195 & 1.581 & 340 & 3.262 & -0.171 & 0.162 & 0.161 & 0.001 & / \\
\hline 400 & heptadecanoic acid & 5.112 & 0.195 & 1.581 & 350 & 3.262 & -0.171 & 0.160 & 0.159 & 0.001 & I \\
\hline 401 & heptadecanoic acid & 5.112 & 0.195 & 1.581 & 360 & 3.262 & -0.171 & 0.159 & 0.157 & 0.002 & / \\
\hline 402 & stearic acid & 5.137 & 0.191 & 1.555 & 330 & 3.306 & -0.176 & 0.176 & 0.165 & 0.011 & l \\
\hline 403 & stearic acid & 5.137 & 0.191 & 1.555 & 340 & 3.306 & -0.176 & 0.174 & 0.162 & 0.012 & / \\
\hline 404 & stearic acid & 5.137 & 0.191 & 1.555 & 350 & 3.306 & -0.176 & 0.172 & 0.160 & 0.012 & 1 \\
\hline 405 & stearic acid & 5.137 & 0.191 & 1.555 & 360 & 3.306 & -0.176 & 0.169 & 0.158 & 0.011 & t \\
\hline 406 & stearic acid & 5.137 & 0.191 & 1.555 & 370 & 3.306 & -0.176 & 0.167 & 0.156 & 0.011 & / \\
\hline 407 & stearic acid & 5.137 & 0.191 & 1.555 & 380 & 3.306 & -0.176 & 0.164 & 0.154 & 0.010 & I \\
\hline 408 & stearic acid & 5.137 & 0.191 & 1.555 & 390 & 3.306 & -0.176 & 0.162 & 0.152 & 0.010 & / \\
\hline 409 & stearic acid & 5.137 & 0.191 & 1.555 & 400 & 3.306 & -0.176 & 0.160 & 0.150 & 0.010 & / \\
\hline 410 & stearic acid & 5.137 & 0.191 & 1.555 & 420 & 3.306 & -0.176 & 0.155 & 0.146 & 0.009 & / \\
\hline 411 & stearic acid & 5.137 & 0.191 & 1.555 & 440 & 3.306 & -0.176 & 0.150 & 0.142 & 0.008 & l \\
\hline 412 & stearic acid & 5.137 & 0.191 & 1.555 & 450 & 3.306 & -0.176 & 0.147 & 0.140 & 0.007 & l \\
\hline 413 & stearic acid & 5.137 & 0.191 & 1.555 & 460 & 3.306 & -0.176 & 0.145 & 0.138 & 0.007 & 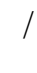 \\
\hline 414 & stearic acid & 5.137 & 0.191 & 1.555 & 470 & 3.306 & -0.176 & 0.142 & 0.136 & 0.006 & 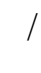 \\
\hline 415 & caprylic acid & 4.850 & 0.264 & 2.000 & 300 & 2.690 & -0.090 & 0.146 & 0.152 & -0.006 & t \\
\hline 416 & caprylic acid & 4.850 & 0.264 & 2.000 & 340 & 2.690 & -0.090 & 0.140 & 0.144 & -0.004 & t \\
\hline 417 & caprylic acid & 4.850 & 0.264 & 2.000 & 380 & 2.690 & -0.090 & 0.134 & 0.136 & -0.002 & I \\
\hline 418 & caprylic acid & 4.850 & 0.264 & 2.000 & 400 & 2.690 & -0.090 & 0.131 & 0.132 & -0.001 & / \\
\hline 419 & caprylic acid & 4.850 & 0.264 & 2.000 & 420 & 2.690 & -0.090 & 0.128 & 0.128 & 0.000 & I \\
\hline
\end{tabular}




\begin{tabular}{|c|c|c|c|c|c|c|c|c|c|c|c|}
\hline 420 & caprylic acid & 4.850 & 0.264 & 2.000 & 440 & 2.690 & -0.090 & 0.125 & 0.124 & 0.001 & I \\
\hline 421 & caprylic acid & 4.850 & 0.264 & 2.000 & 450 & 2.690 & -0.090 & 0.124 & 0.121 & 0.003 & / \\
\hline 422 & nonanoic acid & 4.882 & 0.251 & 1.927 & 300 & 2.777 & -0.107 & 0.150 & 0.154 & -0.004 & I \\
\hline 423 & nonanoic acid & 4.882 & 0.251 & 1.927 & 400 & 2.777 & -0.107 & 0.133 & 0.134 & -0.001 & I \\
\hline 424 & nonanoic acid & 4.882 & 0.251 & 1.927 & 420 & 2.777 & -0.107 & 0.130 & 0.130 & 0.000 & / \\
\hline 425 & nonanoic acid & 4.882 & 0.251 & 1.927 & 440 & 2.777 & -0.107 & 0.127 & 0.126 & 0.001 & I \\
\hline 426 & Methylethylketone & 4.310 & 0.335 & 1.854 & 280 & 1.565 & 0.098 & 0.159 & 0.149 & 0.010 & I \\
\hline 427 & Methylethylketone & 4.310 & 0.335 & 1.854 & 300 & 1.565 & 0.098 & 0.150 & 0.145 & 0.005 & / \\
\hline 428 & Methylethylketone & 4.310 & 0.335 & 1.854 & 320 & 1.565 & 0.098 & 0.141 & 0.141 & 0.000 & I \\
\hline 429 & Methylethylketone & 4.310 & 0.335 & 1.854 & 340 & 1.565 & 0.098 & 0.133 & 0.137 & -0.004 & I \\
\hline 430 & Methylethylketone & 4.310 & 0.335 & 1.854 & 360 & 1.565 & 0.098 & 0.125 & 0.133 & -0.008 & I \\
\hline 431 & 2-pentanone & 4.366 & 0.300 & 1.924 & 290 & 1.693 & 0.022 & 0.144 & 0.140 & 0.004 & I \\
\hline 432 & 2-pentanone & 4.366 & 0.300 & 1.924 & 300 & 1.693 & 0.022 & 0.141 & 0.138 & 0.003 & / \\
\hline 433 & 2-pentanone & 4.366 & 0.300 & 1.924 & 310 & 1.693 & 0.022 & 0.138 & 0.136 & 0.002 & , \\
\hline 434 & 2-pentanone & 4.366 & 0.300 & 1.924 & 320 & 1.693 & 0.022 & 0.136 & 0.134 & 0.002 & / \\
\hline 435 & 2-pentanone & 4.366 & 0.300 & 1.924 & 330 & 1.693 & 0.022 & 0.133 & 0.132 & 0.001 & / \\
\hline 436 & 2-pentanone & 4.366 & 0.300 & 1.924 & 350 & 1.693 & 0.022 & 0.127 & 0.128 & -0.001 & 1 \\
\hline 437 & 2-pentanone & 4.366 & 0.300 & 1.924 & 360 & 1.693 & 0.022 & 0.124 & 0.126 & -0.002 & I \\
\hline 438 & diethyl ketone & 4.366 & 0.300 & 1.674 & 310 & 1.718 & 0.022 & 0.140 & 0.149 & -0.009 & / \\
\hline 439 & diethyl ketone & 4.366 & 0.300 & 1.674 & 330 & 1.718 & 0.022 & 0.134 & 0.144 & -0.010 & 1 \\
\hline 440 & diethyl ketone & 4.366 & 0.300 & 1.674 & 340 & 1.718 & 0.022 & 0.131 & 0.142 & -0.011 & l \\
\hline 441 & diethyl ketone & 4.366 & 0.300 & 1.674 & 350 & 1.718 & 0.022 & 0.128 & 0.140 & -0.012 & I \\
\hline
\end{tabular}




\begin{tabular}{|c|c|c|c|c|c|c|c|c|c|c|c|}
\hline 442 & diethyl ketone & 4.366 & 0.300 & 1.674 & 370 & 1.718 & 0.022 & 0.123 & 0.136 & -0.013 & I \\
\hline 443 & 2-hexanone & 4.418 & 0.275 & 1.878 & 300 & 1.800 & -0.026 & 0.144 & 0.138 & 0.006 & / \\
\hline 444 & 2-hexanone & 4.418 & 0.275 & 1.878 & 320 & 1.800 & -0.026 & 0.135 & 0.134 & 0.001 & I \\
\hline 445 & 2-hexanone & 4.418 & 0.275 & 1.878 & 360 & 1.800 & -0.026 & 0.120 & 0.126 & -0.006 & / \\
\hline 446 & 2-hexanone & 4.418 & 0.275 & 1.878 & 380 & 1.800 & -0.026 & 0.113 & 0.122 & -0.009 & / \\
\hline 447 & 2-hexanone & 4.418 & 0.275 & 1.878 & 400 & 1.800 & -0.026 & 0.106 & 0.118 & -0.012 & 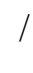 \\
\hline 448 & 2-hexanone & 4.418 & 0.275 & 1.878 & 420 & 1.800 & -0.026 & 0.100 & 0.114 & -0.014 & 1 \\
\hline 449 & 4-heptanone & 4.468 & 0.256 & 1.764 & 340 & 1.975 & -0.060 & 0.127 & 0.134 & -0.007 & / \\
\hline 450 & 4-heptanone & 4.468 & 0.256 & 1.764 & 360 & 1.975 & -0.060 & 0.123 & 0.130 & -0.007 & / \\
\hline 451 & 3-heptanone & 4.468 & 0.256 & 1.764 & 280 & 1.953 & -0.060 & 0.141 & 0.146 & -0.005 & 1 \\
\hline 452 & 3-heptanone & 4.468 & 0.256 & 1.764 & 300 & 1.953 & -0.060 & 0.136 & 0.142 & -0.006 & I \\
\hline 453 & 3-heptanone & 4.468 & 0.256 & 1.764 & 320 & 1.953 & -0.060 & 0.131 & 0.138 & -0.007 & l \\
\hline 454 & 3-heptanone & 4.468 & 0.256 & 1.764 & 340 & 1.953 & -0.060 & 0.127 & 0.134 & -0.007 & / \\
\hline 455 & 3-heptanone & 4.468 & 0.256 & 1.764 & 360 & 1.953 & -0.060 & 0.122 & 0.130 & -0.008 & 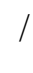 \\
\hline 456 & 3-heptanone & 4.468 & 0.256 & 1.764 & 380 & 1.953 & -0.060 & 0.117 & 0.126 & -0.009 & / \\
\hline 457 & diamyl ketone & 4.646 & 0.213 & 1.624 & 310 & 2.152 & -0.131 & 0.135 & 0.149 & -0.014 & / \\
\hline 458 & diamyl ketone & 4.646 & 0.213 & 1.624 & 330 & 2.152 & -0.131 & 0.131 & 0.145 & -0.014 & / \\
\hline 459 & di-n-hexyl ketone & 4.725 & 0.200 & 1.567 & 320 & 2.523 & -0.149 & 0.138 & 0.148 & -0.010 & / \\
\hline 460 & di-n-hexyl ketone & 4.725 & 0.200 & 1.567 & 350 & 2.523 & -0.149 & 0.132 & 0.142 & -0.010 & / \\
\hline 461 & di-n-hexyl ketone & 4.725 & 0.200 & 1.567 & 360 & 2.523 & -0.149 & 0.130 & 0.140 & -0.010 & I \\
\hline 462 & di-n-hexyl ketone & 4.725 & 0.200 & 1.567 & 370 & 2.523 & -0.149 & 0.128 & 0.138 & -0.010 & l \\
\hline 463 & di-n-hexyl ketone & 4.725 & 0.200 & 1.567 & 380 & 2.523 & -0.149 & 0.126 & 0.136 & -0.010 & I \\
\hline
\end{tabular}




\begin{tabular}{|c|c|c|c|c|c|c|c|c|c|c|c|}
\hline 464 & di-n-hexyl ketone & 4.725 & 0.200 & 1.567 & 410 & 2.523 & -0.149 & 0.120 & 0.129 & -0.009 & I \\
\hline 465 & di-n-hexyl ketone & 4.725 & 0.200 & 1.567 & 420 & 2.523 & -0.149 & 0.118 & 0.127 & -0.009 & l \\
\hline 466 & di-n-hexyl ketone & 4.725 & 0.200 & 1.567 & 430 & 2.523 & -0.149 & 0.116 & 0.125 & -0.009 & I \\
\hline 467 & di-n-hexyl ketone & 4.725 & 0.200 & 1.567 & 450 & 2.523 & -0.149 & 0.112 & 0.121 & -0.009 & / \\
\hline 468 & di-n-hexyl ketone & 4.725 & 0.200 & 1.567 & 460 & 2.523 & -0.149 & 0.110 & 0.119 & -0.009 & 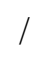 \\
\hline 469 & 2-octanone & 4.516 & 0.242 & 1.764 & 270 & 1.967 & -0.084 & 0.141 & 0.149 & -0.008 & / \\
\hline 470 & 2-octanone & 4.516 & 0.242 & 1.764 & 290 & 1.967 & -0.084 & 0.137 & 0.145 & -0.008 & / \\
\hline 471 & 2-octanone & 4.516 & 0.242 & 1.764 & 300 & 1.967 & -0.084 & 0.135 & 0.142 & -0.007 & t \\
\hline 472 & 2-octanone & 4.516 & 0.242 & 1.764 & 310 & 1.967 & -0.084 & 0.132 & 0.140 & -0.008 & t \\
\hline 473 & 2-octanone & 4.516 & 0.242 & 1.764 & 320 & 1.967 & -0.084 & 0.130 & 0.138 & -0.008 & I \\
\hline 474 & 2-octanone & 4.516 & 0.242 & 1.764 & 330 & 1.967 & -0.084 & 0.128 & 0.136 & -0.008 & / \\
\hline 475 & 2-octanone & 4.516 & 0.242 & 1.764 & 350 & 1.967 & -0.084 & 0.123 & 0.132 & -0.009 & / \\
\hline 476 & 2-octanone & 4.516 & 0.242 & 1.764 & 360 & 1.967 & -0.084 & 0.121 & 0.130 & -0.009 & / \\
\hline 477 & 2-octanone & 4.516 & 0.242 & 1.764 & 380 & 1.967 & -0.084 & 0.116 & 0.126 & -0.010 & 1 \\
\hline 478 & 2-octanone & 4.516 & 0.242 & 1.764 & 390 & 1.967 & -0.084 & 0.114 & 0.124 & -0.010 & 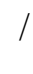 \\
\hline 479 & 2-octanone & 4.516 & 0.242 & 1.764 & 400 & 1.967 & -0.084 & 0.112 & 0.122 & -0.010 & 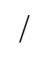 \\
\hline 480 & 2-octanone & 4.516 & 0.242 & 1.764 & 410 & 1.967 & -0.084 & 0.110 & 0.120 & -0.010 & 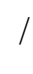 \\
\hline 481 & acetone & 4.251 & 0.390 & 1.571 & 200 & 1.412 & 0.232 & 0.197 & 0.186 & 0.011 & 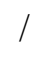 \\
\hline 482 & acetone & 4.251 & 0.390 & 1.571 & 220 & 1.412 & 0.232 & 0.190 & 0.182 & 0.008 & $t$ \\
\hline 483 & acetone & 4.251 & 0.390 & 1.571 & 240 & 1.412 & 0.232 & 0.182 & 0.178 & 0.004 & I \\
\hline 484 & acetone & 4.251 & 0.390 & 1.571 & 260 & 1.412 & 0.232 & 0.174 & 0.174 & 0.000 & 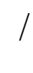 \\
\hline 485 & acetone & 4.251 & 0.390 & 1.571 & 280 & 1.412 & 0.232 & 0.167 & 0.170 & -0.003 & l \\
\hline
\end{tabular}




\begin{tabular}{|c|c|c|c|c|c|c|c|c|c|c|c|}
\hline 486 & acetone & 4.251 & 0.390 & 1.571 & 320 & 1.412 & 0.232 & 0.152 & 0.162 & -0.010 & , \\
\hline 487 & Methyl isopropyl ketone & 4.387 & 0.300 & 1.799 & 193 & 1.799 & 0.159 & 0.170 & 0.158 & 0.011 & I \\
\hline 488 & Methyl isopropyl ketone & 4.387 & 0.300 & 1.799 & 213 & 1.799 & 0.159 & 0.164 & 0.154 & 0.010 & / \\
\hline 489 & Methyl isopropyl ketone & 4.387 & 0.300 & 1.799 & 273 & 1.799 & 0.159 & 0.148 & 0.142 & 0.006 & 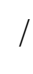 \\
\hline 490 & Methyl isopropyl ketone & 4.387 & 0.300 & 1.799 & 313 & 1.799 & 0.159 & 0.136 & 0.134 & 0.002 & / \\
\hline 491 & Methyl isopropyl ketone & 4.387 & 0.300 & 1.799 & 333 & 1.799 & 0.159 & 0.130 & 0.130 & 0.000 & t \\
\hline 492 & Methyl isopropyl ketone & 4.387 & 0.300 & 1.799 & 353 & 1.799 & 0.159 & 0.123 & 0.126 & -0.002 & \\
\hline 493 & Methyl isopropyl ketone & 4.387 & 0.300 & 1.799 & 373 & 1.799 & 0.159 & 0.117 & 0.122 & -0.005 & \\
\hline 494 & Methyl isobutyl ketone & 4.461 & 0.275 & 1.733 & 193 & 2.058 & 0.319 & 0.163 & 0.147 & 0.016 & \\
\hline 495 & Methyl isobutyl ketone & 4.461 & 0.275 & 1.733 & 213 & 2.058 & 0.319 & 0.158 & 0.143 & 0.015 & \\
\hline 496 & Methyl isobutyl ketone & 4.461 & 0.275 & 1.733 & 233 & 2.058 & 0.319 & 0.153 & 0.139 & 0.014 & \\
\hline 497 & Methyl isobutyl ketone & 4.461 & 0.275 & 1.733 & 293 & 2.058 & 0.319 & 0.138 & 0.127 & 0.011 & 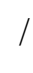 \\
\hline 498 & Methyl isobutyl ketone & 4.461 & 0.275 & 1.733 & 313 & 2.058 & 0.319 & 0.132 & 0.122 & 0.010 & 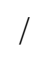 \\
\hline 499 & Methyl isobutyl ketone & 4.461 & 0.275 & 1.733 & 353 & 2.058 & 0.319 & 0.121 & 0.114 & 0.007 & 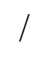 \\
\hline 500 & Methyl isobutyl ketone & 4.461 & 0.275 & 1.733 & 373 & 2.058 & 0.319 & 0.116 & 0.110 & 0.005 & \\
\hline 501 & Ethyl formate & 4.425 & 0.415 & 2.404 & 270 & 2.044 & -0.106 & 0.172 & 0.161 & 0.011 & \\
\hline 502 & Ethyl formate & 4.425 & 0.415 & 2.404 & 280 & 2.044 & -0.106 & 0.168 & 0.159 & 0.009 & \\
\hline 503 & Ethyl formate & 4.425 & 0.415 & 2.404 & 290 & 2.044 & -0.106 & 0.164 & 0.157 & 0.007 & \\
\hline 504 & Ethyl formate & 4.425 & 0.415 & 2.404 & 310 & 2.044 & -0.106 & 0.155 & 0.153 & 0.002 & \\
\hline 505 & Ethyl formate & 4.425 & 0.415 & 2.404 & 320 & 2.044 & -0.106 & 0.151 & 0.151 & 0.000 & / \\
\hline 506 & Ethyl formate & 4.425 & 0.415 & 2.404 & 330 & 2.044 & -0.106 & 0.146 & 0.149 & -0.003 & / \\
\hline 507 & Methyl propionate & 4.485 & 0.362 & 2.093 & 280 & 2.524 & 0.118 & 0.144 & 0.144 & 0.000 & 1 \\
\hline
\end{tabular}




\begin{tabular}{|c|c|c|c|c|c|c|c|c|c|c|c|}
\hline 508 & Methyl propionate & 4.485 & 0.362 & 2.093 & 300 & 2.524 & 0.118 & 0.141 & 0.140 & 0.001 & 1 \\
\hline 509 & Methyl propionate & 4.485 & 0.362 & 2.093 & 320 & 2.524 & 0.118 & 0.137 & 0.136 & 0.001 & / \\
\hline 510 & ethyl acetate & 4.485 & 0.362 & 2.093 & 270 & 2.428 & 0.170 & 0.152 & 0.144 & 0.008 & I \\
\hline 511 & ethyl acetate & 4.485 & 0.362 & 2.093 & 290 & 2.428 & 0.170 & 0.146 & 0.140 & 0.006 & I \\
\hline 512 & ethyl acetate & 4.485 & 0.362 & 2.093 & 300 & 2.428 & 0.170 & 0.143 & 0.138 & 0.005 & / \\
\hline 513 & ethyl acetate & 4.485 & 0.362 & 2.093 & 310 & 2.428 & 0.170 & 0.140 & 0.136 & 0.004 & / \\
\hline 514 & ethyl acetate & 4.485 & 0.362 & 2.093 & 340 & 2.428 & 0.170 & 0.131 & 0.130 & 0.001 & r \\
\hline 515 & ethyl acetate & 4.485 & 0.362 & 2.093 & 350 & 2.428 & 0.170 & 0.128 & 0.128 & 0.000 & 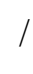 \\
\hline 516 & ethyl acetate & 4.485 & 0.362 & 2.093 & 360 & 2.428 & 0.170 & 0.124 & 0.126 & -0.002 & r \\
\hline 517 & methyl butyrate & 4.532 & 0.326 & 2.133 & 300 & 2.714 & 0.046 & 0.140 & 0.133 & 0.007 & I \\
\hline 518 & ethyl propionate & 4.532 & 0.326 & 2.016 & 320 & 2.640 & 0.088 & 0.134 & 0.133 & 0.001 & I \\
\hline 519 & ethyl propionate & 4.532 & 0.326 & 2.016 & 330 & 2.640 & 0.088 & 0.129 & 0.131 & -0.002 & / \\
\hline 520 & ethyl propionate & 4.532 & 0.326 & 2.016 & 340 & 2.640 & 0.088 & 0.125 & 0.129 & -0.004 & / \\
\hline 521 & butyl formate & 4.522 & 0.326 & 2.292 & 300 & 2.381 & -0.166 & 0.136 & 0.140 & -0.004 & 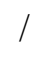 \\
\hline 522 & butyl formate & 4.522 & 0.326 & 2.292 & 320 & 2.381 & -0.166 & 0.131 & 0.136 & -0.005 & 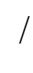 \\
\hline 523 & butyl formate & 4.522 & 0.326 & 2.292 & 340 & 2.381 & -0.166 & 0.126 & 0.132 & -0.006 & 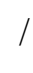 \\
\hline 524 & butyl formate & 4.522 & 0.326 & 2.292 & 360 & 2.381 & -0.166 & 0.121 & 0.128 & -0.007 & 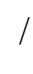 \\
\hline 525 & propyl acetate & 4.532 & 0.326 & 2.133 & 290 & 2.596 & 0.088 & 0.142 & 0.134 & 0.008 & 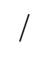 \\
\hline 526 & propyl acetate & 4.532 & 0.326 & 2.133 & 300 & 2.596 & 0.088 & 0.140 & 0.132 & 0.008 & r \\
\hline 527 & propyl acetate & 4.532 & 0.326 & 2.133 & 310 & 2.596 & 0.088 & 0.138 & 0.130 & 0.008 & I \\
\hline 528 & propyl acetate & 4.532 & 0.326 & 2.133 & 340 & 2.596 & 0.088 & 0.131 & 0.123 & 0.008 & / \\
\hline 529 & propyl acetate & 4.532 & 0.326 & 2.133 & 360 & 2.596 & 0.088 & 0.127 & 0.119 & 0.008 & I \\
\hline
\end{tabular}




\begin{tabular}{|c|c|c|c|c|c|c|c|c|c|c|c|}
\hline 530 & propyl acetate & 4.532 & 0.326 & 2.133 & 370 & 2.596 & 0.088 & 0.125 & 0.117 & 0.008 & I \\
\hline 531 & methyl valerate & 4.577 & 0.300 & 2.071 & 280 & 2.872 & -0.001 & 0.142 & 0.136 & 0.006 & / \\
\hline 532 & methyl valerate & 4.577 & 0.300 & 2.071 & 290 & 2.872 & -0.001 & 0.139 & 0.134 & 0.005 & I \\
\hline 533 & methyl valerate & 4.577 & 0.300 & 2.071 & 300 & 2.872 & -0.001 & 0.137 & 0.132 & 0.005 & I \\
\hline 534 & methyl valerate & 4.577 & 0.300 & 2.071 & 310 & 2.872 & -0.001 & 0.135 & 0.130 & 0.005 & l \\
\hline 535 & methyl valerate & 4.577 & 0.300 & 2.071 & 330 & 2.872 & -0.001 & 0.131 & 0.126 & 0.005 & 1 \\
\hline 536 & methyl valerate & 4.577 & 0.300 & 2.071 & 340 & 2.872 & -0.001 & 0.129 & 0.124 & 0.005 & / \\
\hline 537 & butyl acetate & 4.577 & 0.300 & 2.071 & 300 & 2.738 & 0.035 & 0.135 & 0.132 & 0.003 & t \\
\hline 538 & butyl acetate & 4.577 & 0.300 & 2.071 & 310 & 2.738 & 0.035 & 0.132 & 0.130 & 0.002 & / \\
\hline 539 & butyl acetate & 4.577 & 0.300 & 2.071 & 320 & 2.738 & 0.035 & 0.130 & 0.128 & 0.002 & I \\
\hline 540 & butyl acetate & 4.577 & 0.300 & 2.071 & 330 & 2.738 & 0.035 & 0.127 & 0.125 & 0.002 & I \\
\hline 541 & butyl acetate & 4.577 & 0.300 & 2.071 & 340 & 2.738 & 0.035 & 0.124 & 0.123 & 0.001 & I \\
\hline 542 & butyl acetate & 4.577 & 0.300 & 2.071 & 350 & 2.738 & 0.035 & 0.122 & 0.121 & 0.001 & / \\
\hline 543 & butyl acetate & 4.577 & 0.300 & 2.071 & 360 & 2.738 & 0.035 & 0.119 & 0.119 & 0.000 & / \\
\hline 544 & isobutyl acetate & 4.594 & 0.300 & 2.071 & 260 & 2.831 & 0.134 & 0.134 & 0.134 & 0.000 & I \\
\hline 545 & isobutyl acetate & 4.594 & 0.300 & 2.071 & 270 & 2.831 & 0.134 & 0.130 & 0.132 & -0.002 & 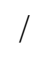 \\
\hline 546 & isobutyl acetate & 4.594 & 0.300 & 2.071 & 280 & 2.831 & 0.134 & 0.126 & 0.130 & -0.004 & 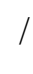 \\
\hline 547 & isobutyl acetate & 4.594 & 0.300 & 2.071 & 310 & 2.831 & 0.134 & 0.116 & 0.124 & -0.008 & 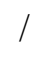 \\
\hline 548 & isobutyl acetate & 4.594 & 0.300 & 2.071 & 330 & 2.831 & 0.134 & 0.109 & 0.120 & -0.011 & I \\
\hline 549 & isobutyl acetate & 4.594 & 0.300 & 2.071 & 340 & 2.831 & 0.134 & 0.106 & 0.118 & -0.012 & I \\
\hline 550 & isobutyl acetate & 4.594 & 0.300 & 2.071 & 350 & 2.831 & 0.134 & 0.103 & 0.116 & -0.013 & , \\
\hline 551 & isobutyl acetate & 4.594 & 0.300 & 2.071 & 360 & 2.831 & 0.134 & 0.100 & 0.114 & -0.014 & I \\
\hline
\end{tabular}




\begin{tabular}{|c|c|c|c|c|c|c|c|c|c|c|c|}
\hline 552 & isobutyl acetate & 4.594 & 0.300 & 2.071 & 370 & 2.831 & 0.134 & 0.098 & 0.112 & -0.014 & I \\
\hline 553 & isobutyl acetate & 4.594 & 0.300 & 2.071 & 380 & 2.831 & 0.134 & 0.095 & 0.110 & -0.015 & I \\
\hline 554 & isobutyl acetate & 4.594 & 0.300 & 2.071 & 390 & 2.831 & 0.134 & 0.094 & 0.108 & -0.014 & I \\
\hline 555 & amyl formate & 4.567 & 0.300 & 2.181 & 300 & 2.512 & -0.181 & 0.134 & 0.141 & -0.007 & , \\
\hline 556 & amyl formate & 4.567 & 0.300 & 2.181 & 360 & 2.512 & -0.181 & 0.123 & 0.129 & -0.006 & l \\
\hline 557 & ethyl butyrate & 4.577 & 0.300 & 2.071 & 280 & 2.816 & 0.035 & 0.141 & 0.135 & 0.006 & 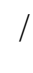 \\
\hline 558 & ethyl butyrate & 4.577 & 0.300 & 2.071 & 290 & 2.816 & 0.035 & 0.138 & 0.133 & 0.005 & / \\
\hline 559 & ethyl butyrate & 4.577 & 0.300 & 2.071 & 300 & 2.816 & 0.035 & 0.136 & 0.131 & 0.005 & 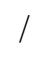 \\
\hline 560 & ethyl butyrate & 4.577 & 0.300 & 2.071 & 310 & 2.816 & 0.035 & 0.134 & 0.129 & 0.005 & r \\
\hline 561 & ethyl butyrate & 4.577 & 0.300 & 2.071 & 320 & 2.816 & 0.035 & 0.132 & 0.127 & 0.005 & I \\
\hline 562 & ethyl butyrate & 4.577 & 0.300 & 2.071 & 330 & 2.816 & 0.035 & 0.129 & 0.124 & 0.005 & I \\
\hline 563 & ethyl butyrate & 4.577 & 0.300 & 2.071 & 340 & 2.816 & 0.035 & 0.128 & 0.122 & 0.006 & I \\
\hline 564 & n-propyl propionate & 4.577 & 0.300 & 2.071 & 320 & 2.808 & 0.035 & 0.134 & 0.127 & 0.007 & / \\
\hline 565 & methyl capronate & 4.620 & 0.280 & 1.999 & 280 & 3.007 & -0.035 & 0.139 & 0.137 & 0.002 & I \\
\hline 566 & methyl capronate & 4.620 & 0.280 & 1.999 & 300 & 3.007 & -0.035 & 0.135 & 0.133 & 0.002 & 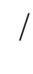 \\
\hline 567 & methyl capronate & 4.620 & 0.280 & 1.999 & 320 & 3.007 & -0.035 & 0.131 & 0.129 & 0.002 & 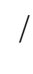 \\
\hline 568 & methyl capronate & 4.620 & 0.280 & 1.999 & 340 & 3.007 & -0.035 & 0.127 & 0.125 & 0.002 & 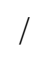 \\
\hline 569 & methyl capronate & 4.620 & 0.280 & 1.999 & 360 & 3.007 & -0.035 & 0.123 & 0.121 & 0.002 & 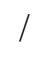 \\
\hline 570 & methyl capronate & 4.620 & 0.280 & 1.999 & 380 & 3.007 & -0.035 & 0.119 & 0.117 & 0.002 & 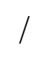 \\
\hline 571 & n-amyl acetate & 4.620 & 0.280 & 1.999 & 320 & 2.858 & -0.004 & 0.127 & 0.129 & -0.002 & I \\
\hline 572 & n-amyl acetate & 4.620 & 0.280 & 1.999 & 340 & 2.858 & -0.004 & 0.122 & 0.125 & -0.003 & r \\
\hline 573 & n-amyl acetate & 4.620 & 0.280 & 1.999 & 360 & 2.858 & -0.004 & 0.116 & 0.121 & -0.005 & / \\
\hline
\end{tabular}




\begin{tabular}{|c|c|c|c|c|c|c|c|c|c|c|c|}
\hline 574 & n-amyl acetate & 4.620 & 0.280 & 1.999 & 380 & 2.858 & -0.004 & 0.111 & 0.117 & -0.006 & 1 \\
\hline 575 & n-butyl propionate & 4.620 & 0.280 & 2.032 & 320 & 2.949 & -0.004 & 0.134 & 0.126 & 0.008 & / \\
\hline 576 & n-butyl propionate & 4.620 & 0.280 & 2.032 & 340 & 2.949 & -0.004 & 0.128 & 0.122 & 0.006 & I \\
\hline 577 & n-butyl propionate & 4.620 & 0.280 & 2.032 & 360 & 2.949 & -0.004 & 0.124 & 0.118 & 0.006 & / \\
\hline 578 & n-butyl propionate & 4.620 & 0.280 & 2.032 & 380 & 2.949 & -0.004 & 0.119 & 0.114 & 0.005 & / \\
\hline 579 & n-butyl propionate & 4.620 & 0.280 & 2.032 & 400 & 2.949 & -0.004 & 0.114 & 0.110 & 0.004 & / \\
\hline 580 & n-butyl propionate & 4.620 & 0.280 & 2.032 & 420 & 2.949 & -0.004 & 0.110 & 0.106 & 0.004 & r \\
\hline 581 & isoamyl acetate & 4.636 & 0.280 & 2.119 & 270 & 2.926 & 0.083 & 0.136 & 0.128 & 0.008 & 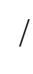 \\
\hline 582 & isoamyl acetate & 4.636 & 0.280 & 2.119 & 290 & 2.926 & 0.083 & 0.131 & 0.124 & 0.007 & \\
\hline 583 & isoamyl acetate & 4.636 & 0.280 & 2.119 & 310 & 2.926 & 0.083 & 0.127 & 0.120 & 0.007 & 1 \\
\hline 584 & isoamyl acetate & 4.636 & 0.280 & 2.119 & 330 & 2.926 & 0.083 & 0.122 & 0.116 & 0.006 & 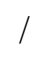 \\
\hline 585 & isoamyl acetate & 4.636 & 0.280 & 2.119 & 350 & 2.926 & 0.083 & 0.118 & 0.112 & 0.006 & 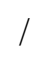 \\
\hline 586 & isoamyl acetate & 4.636 & 0.280 & 2.119 & 360 & 2.926 & 0.083 & 0.115 & 0.110 & 0.005 & I \\
\hline 587 & n-hexyl formate & 4.610 & 0.280 & 2.084 & 300 & 2.626 & -0.191 & 0.140 & 0.142 & -0.002 & 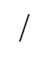 \\
\hline 588 & n-hexyl formate & 4.610 & 0.280 & 2.084 & 320 & 2.626 & -0.191 & 0.134 & 0.138 & -0.004 & 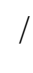 \\
\hline 589 & n-hexyl formate & 4.610 & 0.280 & 2.084 & 360 & 2.626 & -0.191 & 0.123 & 0.130 & -0.007 & \\
\hline 590 & n-hexyl formate & 4.610 & 0.280 & 2.084 & 380 & 2.626 & -0.191 & 0.117 & 0.126 & -0.009 & 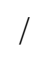 \\
\hline 591 & n-hexyl formate & 4.610 & 0.280 & 2.084 & 400 & 2.626 & -0.191 & 0.112 & 0.122 & -0.010 & 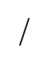 \\
\hline 592 & n-hexyl butyrate & 4.738 & 0.240 & 1.873 & 320 & 3.351 & -0.074 & 0.133 & 0.130 & 0.003 & ? \\
\hline 593 & n-hexyl butyrate & 4.738 & 0.240 & 1.873 & 360 & 3.351 & -0.074 & 0.124 & 0.122 & 0.002 & I \\
\hline 594 & n-hexyl butyrate & 4.738 & 0.240 & 1.873 & 380 & 3.351 & -0.074 & 0.120 & 0.118 & 0.002 & / \\
\hline 595 & n-hexyl butyrate & 4.738 & 0.240 & 1.873 & 400 & 3.351 & -0.074 & 0.116 & 0.114 & 0.002 & 1 \\
\hline
\end{tabular}




\begin{tabular}{|c|c|c|c|c|c|c|c|c|c|c|c|}
\hline 596 & n-hexyl butyrate & 4.738 & 0.240 & 1.873 & 440 & 3.351 & -0.074 & 0.107 & 0.106 & 0.001 & I \\
\hline 597 & n-hexyl butyrate & 4.738 & 0.240 & 1.873 & 460 & 3.351 & -0.074 & 0.103 & 0.102 & 0.001 & I \\
\hline 598 & n-heptyl propionate & 4.738 & 0.240 & 1.873 & 320 & 3.268 & -0.074 & 0.133 & 0.131 & 0.002 & I \\
\hline 599 & $\mathrm{n}$-heptyl propionate & 4.738 & 0.240 & 1.873 & 340 & 3.268 & -0.074 & 0.128 & 0.127 & 0.001 & / \\
\hline 600 & n-heptyl propionate & 4.738 & 0.240 & 1.873 & 380 & 3.268 & -0.074 & 0.120 & 0.119 & 0.001 & I \\
\hline 601 & n-heptyl propionate & 4.738 & 0.240 & 1.873 & 400 & 3.268 & -0.074 & 0.117 & 0.115 & 0.002 & / \\
\hline 602 & n-octyl acetate & 4.738 & 0.240 & 1.814 & 310 & 3.139 & -0.074 & 0.142 & 0.138 & 0.004 & 1 \\
\hline 603 & n-octyl acetate & 4.738 & 0.240 & 1.814 & 320 & 3.139 & -0.074 & 0.138 & 0.136 & 0.002 & t \\
\hline 604 & n-octyl acetate & 4.738 & 0.240 & 1.814 & 340 & 3.139 & -0.074 & 0.130 & 0.132 & -0.002 & / \\
\hline 605 & n-octyl propionate & 4.775 & 0.230 & 1.826 & 300 & 3.350 & -0.089 & 0.135 & 0.137 & -0.002 & I \\
\hline 606 & n-octyl propionate & 4.775 & 0.230 & 1.826 & 320 & 3.350 & -0.089 & 0.130 & 0.133 & -0.003 & / \\
\hline 607 & n-octyl propionate & 4.775 & 0.230 & 1.826 & 340 & 3.350 & -0.089 & 0.126 & 0.129 & -0.003 & / \\
\hline 608 & n-octyl propionate & 4.775 & 0.230 & 1.826 & 420 & 3.350 & -0.089 & 0.111 & 0.113 & -0.002 & / \\
\hline 609 & heptylbutanoate & 4.775 & 0.230 & 1.826 & 300 & 3.444 & -0.089 & 0.139 & 0.136 & 0.003 & l \\
\hline 610 & heptylbutanoate & 4.775 & 0.230 & 1.826 & 320 & 3.444 & -0.089 & 0.135 & 0.132 & 0.003 & 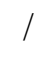 \\
\hline 611 & heptylbutanoate & 4.775 & 0.230 & 1.826 & 340 & 3.444 & -0.089 & 0.130 & 0.128 & 0.002 & t \\
\hline 612 & heptylbutanoate & 4.775 & 0.230 & 1.826 & 360 & 3.444 & -0.089 & 0.126 & 0.124 & 0.002 & 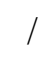 \\
\hline 613 & heptylbutanoate & 4.775 & 0.230 & 1.826 & 380 & 3.444 & -0.089 & 0.122 & 0.120 & 0.002 & 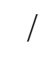 \\
\hline 614 & heptylbutanoate & 4.775 & 0.230 & 1.826 & 400 & 3.444 & -0.089 & 0.118 & 0.115 & 0.003 & $t$ \\
\hline 615 & heptylbutanoate & 4.775 & 0.230 & 1.826 & 420 & 3.444 & -0.089 & 0.113 & 0.111 & 0.002 & I \\
\hline 616 & heptylbutanoate & 4.775 & 0.230 & 1.826 & 440 & 3.444 & -0.089 & 0.109 & 0.107 & 0.002 & I \\
\hline 617 & heptylbutanoate & 4.775 & 0.230 & 1.826 & 460 & 3.444 & -0.089 & 0.105 & 0.103 & 0.002 & / \\
\hline
\end{tabular}




\begin{tabular}{|c|c|c|c|c|c|c|c|c|c|c|c|}
\hline 618 & heptylbutanoate & 4.775 & 0.230 & 1.826 & 480 & 3.444 & -0.089 & 0.100 & 0.099 & 0.001 & , \\
\hline 619 & n-decyl acetate & 4.810 & 0.222 & 1.721 & 310 & 3.283 & -0.102 & 0.142 & 0.142 & 0.000 & / \\
\hline 620 & n-decyl acetate & 4.810 & 0.222 & 1.721 & 320 & 3.283 & -0.102 & 0.138 & 0.140 & -0.002 & / \\
\hline 621 & n-decyl acetate & 4.810 & 0.222 & 1.721 & 330 & 3.283 & -0.102 & 0.134 & 0.138 & -0.004 & I \\
\hline 622 & n-decyl acetate & 4.810 & 0.222 & 1.721 & 340 & 3.283 & -0.102 & 0.130 & 0.136 & -0.006 & / \\
\hline 623 & n-octyl butyrate & 4.810 & 0.222 & 1.782 & 300 & 3.527 & -0.102 & 0.139 & 0.138 & 0.001 & / \\
\hline 624 & n-octyl butyrate & 4.810 & 0.222 & 1.782 & 360 & 3.527 & -0.102 & 0.127 & 0.126 & 0.001 & \\
\hline 625 & n-octyl butyrate & 4.810 & 0.222 & 1.782 & 380 & 3.527 & -0.102 & 0.124 & 0.122 & 0.002 & 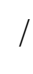 \\
\hline 626 & n-octyl butyrate & 4.810 & 0.222 & 1.782 & 400 & 3.527 & -0.102 & 0.120 & 0.118 & 0.002 & \\
\hline 627 & n-octyl butyrate & 4.810 & 0.222 & 1.782 & 420 & 3.527 & -0.102 & 0.116 & 0.113 & 0.003 & 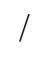 \\
\hline 628 & n-octyl butyrate & 4.810 & 0.222 & 1.782 & 440 & 3.527 & -0.102 & 0.112 & 0.109 & 0.003 & 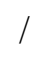 \\
\hline 629 & n-octyl butyrate & 4.810 & 0.222 & 1.782 & 460 & 3.527 & -0.102 & 0.109 & 0.105 & 0.004 & 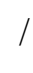 \\
\hline 630 & n-octyl butyrate & 4.810 & 0.222 & 1.782 & 480 & 3.527 & -0.102 & 0.105 & 0.101 & 0.004 & 1 \\
\hline 631 & methyl stearate & 5.027 & 0.187 & 1.509 & 350 & 3.882 & -0.167 & 0.148 & 0.146 & 0.002 & 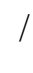 \\
\hline 632 & methyl stearate & 5.027 & 0.187 & 1.509 & 370 & 3.882 & -0.167 & 0.145 & 0.142 & 0.003 & 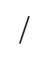 \\
\hline 633 & methyl stearate & 5.027 & 0.187 & 1.509 & 410 & 3.882 & -0.167 & 0.139 & 0.134 & 0.005 & 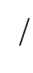 \\
\hline 634 & methyl stearate & 5.027 & 0.187 & 1.509 & 420 & 3.882 & -0.167 & 0.137 & 0.132 & 0.005 & 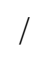 \\
\hline 635 & methyl stearate & 5.027 & 0.187 & 1.509 & 440 & 3.882 & -0.167 & 0.134 & 0.128 & 0.006 & 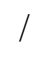 \\
\hline 636 & methyl stearate & 5.027 & 0.187 & 1.509 & 450 & 3.882 & -0.167 & 0.132 & 0.126 & 0.006 & r \\
\hline 637 & n-octadecyl acetate & 5.054 & 0.184 & 1.488 & 340 & 3.686 & -0.159 & 0.167 & 0.152 & 0.015 & I \\
\hline 638 & n-octadecyl acetate & 5.054 & 0.184 & 1.488 & 380 & 3.686 & -0.159 & 0.156 & 0.144 & 0.012 & / \\
\hline 639 & n-butyl stearate & 5.107 & 0.178 & 1.504 & 310 & 4.180 & -0.167 & 0.155 & 0.154 & 0.001 & I \\
\hline
\end{tabular}




\begin{tabular}{|c|c|c|c|c|c|c|c|c|c|c|c|}
\hline 640 & n-butyl stearate & 5.107 & 0.178 & 1.504 & 340 & 4.180 & -0.167 & 0.150 & 0.147 & 0.003 & 1 \\
\hline 641 & n-butyl stearate & 5.107 & 0.178 & 1.504 & 350 & 4.180 & -0.167 & 0.149 & 0.145 & 0.004 & / \\
\hline 642 & n-butyl stearate & 5.107 & 0.178 & 1.504 & 360 & 4.180 & -0.167 & 0.147 & 0.143 & 0.004 & I \\
\hline 643 & n-butyl stearate & 5.107 & 0.178 & 1.504 & 370 & 4.180 & -0.167 & 0.146 & 0.141 & 0.005 & / \\
\hline 644 & n-butyl stearate & 5.107 & 0.178 & 1.504 & 380 & 4.180 & -0.167 & 0.144 & 0.139 & 0.005 & I \\
\hline 645 & n-butyl stearate & 5.107 & 0.178 & 1.504 & 390 & 4.180 & -0.167 & 0.142 & 0.137 & 0.005 & / \\
\hline 646 & n-butyl stearate & 5.107 & 0.178 & 1.504 & 400 & 4.180 & -0.167 & 0.141 & 0.135 & 0.006 & I \\
\hline 647 & n-butyl stearate & 5.107 & 0.178 & 1.504 & 410 & 4.180 & -0.167 & 0.139 & 0.133 & 0.006 & / \\
\hline 648 & n-butyl stearate & 5.107 & 0.178 & 1.504 & 420 & 4.180 & -0.167 & 0.138 & 0.131 & 0.007 & I \\
\hline 649 & n-butyl stearate & 5.107 & 0.178 & 1.504 & 430 & 4.180 & -0.167 & 0.136 & 0.129 & 0.007 & / \\
\hline 650 & n-butyl stearate & 5.107 & 0.178 & 1.504 & 450 & 4.180 & -0.167 & 0.133 & 0.125 & 0.008 & 1 \\
\hline 651 & n-butyl stearate & 5.107 & 0.178 & 1.504 & 460 & 4.180 & -0.167 & 0.131 & 0.123 & 0.008 & / \\
\hline 652 & n-amyl propionate & 4.700 & 0.251 & 1.925 & 300 & 3.246 & -0.056 & 0.138 & 0.132 & 0.006 & / \\
\hline 653 & n-amyl propionate & 4.700 & 0.251 & 1.925 & 326 & 3.246 & -0.056 & 0.132 & 0.127 & 0.005 & / \\
\hline 654 & n-amyl propionate & 4.700 & 0.251 & 1.925 & 359 & 3.246 & -0.056 & 0.126 & 0.120 & 0.006 & / \\
\hline 655 & n-hexyl acetate & 4.661 & 0.264 & 1.931 & 300 & 2.963 & -0.033 & 0.135 & 0.135 & 0.000 & / \\
\hline 656 & n-hexyl acetate & 4.661 & 0.264 & 1.931 & 320 & 2.963 & -0.033 & 0.129 & 0.131 & -0.002 & / \\
\hline 657 & n-hexyl acetate & 4.661 & 0.264 & 1.931 & 340 & 2.963 & -0.033 & 0.124 & 0.127 & -0.003 & / \\
\hline 658 & n-hexyl acetate & 4.661 & 0.264 & 1.931 & 360 & 2.963 & -0.033 & 0.120 & 0.123 & -0.003 & / \\
\hline 659 & n-hexyl acetate & 4.661 & 0.264 & 1.931 & 400 & 2.963 & -0.033 & 0.112 & 0.115 & -0.003 & I \\
\hline 660 & n-hexyl acetate & 4.661 & 0.264 & 1.931 & 420 & 2.963 & -0.033 & 0.108 & 0.111 & -0.003 & l \\
\hline 661 & n-heptyl formate & 4.652 & 0.264 & 2.000 & 270 & 2.727 & -0.199 & 0.142 & 0.150 & -0.008 & I \\
\hline
\end{tabular}




\begin{tabular}{|c|c|c|c|c|c|c|c|c|c|c|c|}
\hline 662 & n-heptyl formate & 4.652 & 0.264 & 2.000 & 290 & 2.727 & -0.199 & 0.139 & 0.146 & -0.007 & , \\
\hline 663 & n-heptyl formate & 4.652 & 0.264 & 2.000 & 330 & 2.727 & -0.199 & 0.132 & 0.138 & -0.006 & / \\
\hline 664 & n-heptyl formate & 4.652 & 0.264 & 2.000 & 350 & 2.727 & -0.199 & 0.128 & 0.134 & -0.006 & / \\
\hline 665 & n-heptyl formate & 4.652 & 0.264 & 2.000 & 390 & 2.727 & -0.199 & 0.121 & 0.125 & -0.004 & I \\
\hline 666 & n-heptyl formate & 4.652 & 0.264 & 2.000 & 410 & 2.727 & -0.199 & 0.117 & 0.121 & -0.004 & / \\
\hline 667 & n-heptyl formate & 4.652 & 0.264 & 2.000 & 430 & 2.727 & -0.199 & 0.114 & 0.117 & -0.003 & / \\
\hline 668 & isoamyl propionate & 4.677 & 0.264 & 2.056 & 360 & 3.138 & 0.045 & 0.118 & 0.111 & 0.007 & \\
\hline 669 & isoamyl propionate & 4.677 & 0.264 & 2.056 & 380 & 3.138 & 0.045 & 0.115 & 0.107 & 0.008 & 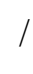 \\
\hline 670 & isoamyl propionate & 4.677 & 0.264 & 2.056 & 420 & 3.138 & 0.045 & 0.108 & 0.099 & 0.009 & t \\
\hline 671 & Ethyl hexanoate & 4.661 & 0.264 & 1.979 & 300 & 3.087 & -0.033 & 0.137 & 0.131 & 0.006 & , \\
\hline 672 & Ethyl hexanoate & 4.661 & 0.264 & 1.979 & 320 & 3.087 & -0.033 & 0.133 & 0.127 & 0.006 & 1 \\
\hline 673 & Ethyl hexanoate & 4.661 & 0.264 & 1.979 & 340 & 3.087 & -0.033 & 0.129 & 0.123 & 0.006 & 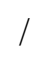 \\
\hline 674 & Ethyl hexanoate & 4.661 & 0.264 & 1.979 & 400 & 3.087 & -0.033 & 0.118 & 0.111 & 0.007 & 1 \\
\hline 675 & n-amylbutyrate & 4.700 & 0.251 & 1.925 & 320 & 3.246 & -0.056 & 0.135 & 0.128 & 0.007 & 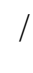 \\
\hline 676 & n-amylbutyrate & 4.700 & 0.251 & 1.925 & 340 & 3.246 & -0.056 & 0.131 & 0.124 & 0.007 & \\
\hline 677 & n-amylbutyrate & 4.700 & 0.251 & 1.925 & 390 & 3.246 & -0.056 & 0.122 & 0.114 & 0.008 & \\
\hline 678 & n-hexyl propionate & 4.700 & 0.251 & 1.925 & 280 & 3.175 & -0.056 & 0.139 & 0.137 & 0.002 & \\
\hline 679 & n-hexyl propionate & 4.700 & 0.251 & 1.925 & 360 & 3.175 & -0.056 & 0.126 & 0.121 & 0.005 & \\
\hline 680 & n-hexyl propionate & 4.700 & 0.251 & 1.925 & 380 & 3.175 & -0.056 & 0.123 & 0.117 & 0.006 & \\
\hline 681 & n-hexyl propionate & 4.700 & 0.251 & 1.925 & 400 & 3.175 & -0.056 & 0.120 & 0.113 & 0.007 & I \\
\hline 682 & n-hexyl propionate & 4.700 & 0.251 & 1.925 & 420 & 3.175 & -0.056 & 0.116 & 0.109 & 0.007 & / \\
\hline 683 & n-hexyl propionate & 4.700 & 0.251 & 1.925 & 430 & 3.175 & -0.056 & 0.115 & 0.107 & 0.008 & / \\
\hline
\end{tabular}




\begin{tabular}{|c|c|c|c|c|c|c|c|c|c|c|c|c|}
\hline & 684 & n-octyl formate & 4.691 & 0.251 & 1.927 & 300 & 2.818 & -0.205 & 0.135 & 0.146 & -0.011 & 1 \\
\hline & 685 & n-octyl formate & 4.691 & 0.251 & 1.927 & 320 & 2.818 & -0.205 & 0.131 & 0.142 & -0.011 & I \\
\hline & 686 & n-octyl formate & 4.691 & 0.251 & 1.927 & 360 & 2.818 & -0.205 & 0.122 & 0.133 & -0.011 & 1 \\
\hline & 687 & n-octyl formate & 4.691 & 0.251 & 1.927 & 380 & 2.818 & -0.205 & 0.119 & 0.129 & -0.010 & 1 \\
\hline & 688 & n-octyl formate & 4.691 & 0.251 & 1.927 & 400 & 2.818 & -0.205 & 0.115 & 0.125 & -0.010 & / \\
\hline & 689 & n-heptylacetate & 4.700 & 0.251 & 1.869 & 300 & 3.056 & -0.056 & 0.134 & 0.137 & -0.003 & I \\
\hline & 690 & n-heptylacetate & 4.700 & 0.251 & 1.869 & 320 & 3.056 & -0.056 & 0.129 & 0.133 & -0.004 & I \\
\hline & 691 & n-heptylacetate & 4.700 & 0.251 & 1.869 & 360 & 3.056 & -0.056 & 0.119 & 0.125 & -0.006 & / \\
\hline & 692 & n-heptylacetate & 4.700 & 0.251 & 1.869 & 400 & 3.056 & -0.056 & 0.110 & 0.117 & -0.007 & / \\
\hline & 693 & n-heptylacetate & 4.700 & 0.251 & 1.869 & 420 & 3.056 & -0.056 & 0.106 & 0.113 & -0.007 & I \\
\hline & 694 & n-heptylacetate & 4.700 & 0.251 & 1.869 & 440 & 3.056 & -0.056 & 0.102 & 0.109 & -0.007 & l \\
\hline \multirow[t]{11}{*}{ Test set } & 695 & Methane & 3.771 & 0.484 & 1.792 & 220 & 0.456 & -0.408 & 0.229 & 0.215 & 0.014 & Moderate \\
\hline & 696 & Methane & 3.771 & 0.484 & 1.792 & 240 & 0.456 & -0.408 & 0.222 & 0.211 & 0.011 & Moderate \\
\hline & 697 & Methane & 3.771 & 0.484 & 1.792 & 260 & 0.456 & -0.408 & 0.215 & 0.207 & 0.008 & Moderate \\
\hline & 698 & Methane & 3.771 & 0.484 & 1.792 & 320 & 0.456 & -0.408 & 0.196 & 0.195 & 0.001 & Good \\
\hline & 699 & Methane & 3.771 & 0.484 & 1.792 & 340 & 0.456 & -0.408 & 0.191 & 0.191 & 0.000 & Good \\
\hline & 700 & Methane & 3.771 & 0.484 & 1.792 & 360 & 0.456 & -0.408 & 0.186 & 0.187 & -0.001 & Good \\
\hline & 701 & Methane & 3.771 & 0.484 & 1.792 & 420 & 0.456 & -0.408 & 0.172 & 0.175 & -0.003 & Good \\
\hline & 702 & Methane & 3.771 & 0.484 & 1.792 & 460 & 0.456 & -0.408 & 0.165 & 0.167 & -0.002 & Good \\
\hline & 703 & Methane & 3.771 & 0.484 & 1.792 & 290 & 0.456 & -0.408 & 0.205 & 0.201 & 0.004 & Good \\
\hline & 704 & Ethanol & 3.873 & 0.386 & 1.880 & 320 & 0.527 & -0.257 & 0.160 & 0.159 & 0.001 & Good \\
\hline & 705 & Ethanol & 3.873 & 0.386 & 1.880 & 340 & 0.527 & -0.257 & 0.155 & 0.155 & 0.000 & Good \\
\hline
\end{tabular}




\begin{tabular}{|c|c|c|c|c|c|c|c|c|c|c|c|}
\hline 706 & Ethanol & 3.873 & 0.386 & 1.880 & 360 & 0.527 & -0.257 & 0.151 & 0.151 & 0.000 & Good \\
\hline 707 & n-Propyl alcohol & 3.958 & 0.332 & 1.947 & 180 & 0.605 & -0.258 & 0.180 & 0.172 & 0.008 & Good \\
\hline 708 & n-Propyl alcohol & 3.958 & 0.332 & 1.947 & 200 & 0.605 & -0.258 & 0.176 & 0.168 & 0.008 & Good \\
\hline 709 & n-Propyl alcohol & 3.958 & 0.332 & 1.947 & 340 & 0.605 & -0.258 & 0.146 & 0.140 & 0.006 & Good \\
\hline 710 & n-Propyl alcohol & 3.958 & 0.332 & 1.947 & 360 & 0.605 & -0.258 & 0.142 & 0.136 & 0.006 & Good \\
\hline 711 & Isopropyl alcohol & 3.990 & 0.332 & 1.781 & 190 & 0.640 & 0.005 & 0.161 & 0.165 & -0.004 & Good \\
\hline 712 & Isopropyl alcohol & 3.990 & 0.332 & 1.781 & 200 & 0.640 & 0.005 & 0.159 & 0.163 & -0.004 & Good \\
\hline 713 & Isopropyl alcohol & 3.990 & 0.332 & 1.781 & 260 & 0.640 & 0.005 & 0.146 & 0.151 & -0.005 & Good \\
\hline 714 & Isopropyl alcohol & 3.990 & 0.332 & 1.781 & 300 & 0.640 & 0.005 & 0.138 & 0.143 & -0.005 & Good \\
\hline 715 & Isopropyl alcohol & 3.990 & 0.332 & 1.781 & 380 & 0.640 & 0.005 & 0.125 & 0.127 & -0.002 & Good \\
\hline 716 & n-butanol & 4.036 & 0.297 & 1.872 & 380 & 0.669 & -0.258 & 0.136 & 0.131 & 0.005 & Good \\
\hline 717 & isobutyl alcohol & 4.065 & 0.297 & 1.872 & 220 & 0.722 & -0.106 & 0.146 & 0.155 & -0.009 & Good \\
\hline 718 & isobutyl alcohol & 4.065 & 0.297 & 1.872 & 280 & 0.722 & -0.106 & 0.137 & 0.143 & -0.006 & Good \\
\hline 719 & isobutyl alcohol & 4.065 & 0.297 & 1.872 & 290 & 0.722 & -0.106 & 0.135 & 0.141 & -0.006 & Good \\
\hline 720 & isobutyl alcohol & 4.065 & 0.297 & 1.872 & 300 & 0.722 & -0.106 & 0.134 & 0.139 & -0.005 & Good \\
\hline 721 & tertbutyl alcohol & 4.098 & 0.297 & 1.688 & 320 & 0.772 & 0.309 & 0.110 & 0.122 & -0.012 & Good \\
\hline 722 & tertbutyl alcohol & 4.098 & 0.297 & 1.688 & 350 & 0.772 & 0.309 & 0.109 & 0.116 & -0.007 & Good \\
\hline 723 & tertbutyl alcohol & 4.098 & 0.297 & 1.688 & 370 & 0.772 & 0.309 & 0.108 & 0.112 & -0.004 & Good \\
\hline 724 & tertbutyl alcohol & 4.098 & 0.297 & 1.688 & 410 & 0.772 & 0.309 & 0.104 & 0.104 & 0.000 & Good \\
\hline 725 & n-amyl alcohol & 4.108 & 0.272 & 1.792 & 420 & 0.723 & -0.258 & 0.128 & 0.124 & 0.004 & Good \\
\hline 726 & isoamyl alcohol & 4.136 & 0.272 & 1.945 & 160 & 0.758 & -0.132 & 0.152 & 0.162 & -0.010 & Good \\
\hline 727 & isoamyl alcohol & 4.136 & 0.272 & 1.945 & 220 & 0.758 & -0.132 & 0.146 & 0.150 & -0.004 & Good \\
\hline
\end{tabular}




\begin{tabular}{|c|c|c|c|c|c|c|c|c|c|c|c|}
\hline 728 & isoamyl alcohol & 4.136 & 0.272 & 1.945 & 260 & 0.758 & -0.132 & 0.140 & 0.142 & -0.002 & Good \\
\hline 729 & isoamyl alcohol & 4.136 & 0.272 & 1.945 & 300 & 0.758 & -0.132 & 0.134 & 0.134 & 0.000 & Good \\
\hline 730 & isoamyl alcohol & 4.136 & 0.272 & 1.945 & 440 & 0.758 & -0.132 & 0.111 & 0.106 & 0.005 & Good \\
\hline 731 & 1-hexanol & 4.176 & 0.254 & 1.721 & 300 & 0.768 & -0.257 & 0.152 & 0.150 & 0.002 & Good \\
\hline 732 & 1-hexanol & 4.176 & 0.254 & 1.721 & 400 & 0.768 & -0.257 & 0.132 & 0.130 & 0.002 & Good \\
\hline 733 & 1-hexanol & 4.176 & 0.254 & 1.721 & 480 & 0.768 & -0.257 & 0.113 & 0.114 & -0.001 & Good \\
\hline 734 & 1-hexanol & 4.176 & 0.254 & 1.721 & 540 & 0.768 & -0.257 & 0.099 & 0.102 & -0.004 & Good \\
\hline 735 & 1-hexanol & 4.176 & 0.254 & 1.721 & 570 & 0.768 & -0.257 & 0.091 & 0.096 & -0.005 & Good \\
\hline 736 & 2-hexanol & 4.202 & 0.254 & 1.780 & 240 & 0.819 & -0.111 & 0.148 & 0.152 & -0.004 & Good \\
\hline 737 & 2-hexanol & 4.202 & 0.254 & 1.780 & 260 & 0.819 & -0.111 & 0.145 & 0.148 & -0.003 & Good \\
\hline 738 & 2-hexanol & 4.202 & 0.254 & 1.780 & 300 & 0.819 & -0.111 & 0.137 & 0.140 & -0.003 & Good \\
\hline 739 & 2-hexanol & 4.202 & 0.254 & 1.780 & 320 & 0.819 & -0.111 & 0.134 & 0.136 & -0.002 & Good \\
\hline 740 & 2-hexanol & 4.202 & 0.254 & 1.780 & 340 & 0.819 & -0.111 & 0.131 & 0.132 & -0.001 & Good \\
\hline 741 & 1-heptanol & 4.239 & 0.240 & 1.659 & 340 & 0.808 & -0.257 & 0.146 & 0.145 & 0.001 & Good \\
\hline 742 & 1-heptanol & 4.239 & 0.240 & 1.659 & 380 & 0.808 & -0.257 & 0.137 & 0.137 & 0.000 & Good \\
\hline 743 & 1-heptanol & 4.239 & 0.240 & 1.659 & 420 & 0.808 & -0.257 & 0.128 & 0.129 & -0.001 & Good \\
\hline 744 & 1-heptanol & 4.239 & 0.240 & 1.659 & 440 & 0.808 & -0.257 & 0.124 & 0.125 & -0.002 & Good \\
\hline 745 & 1-heptanol & 4.239 & 0.240 & 1.659 & 460 & 0.808 & -0.257 & 0.120 & 0.121 & -0.002 & Good \\
\hline 746 & 1-heptanol & 4.239 & 0.240 & 1.659 & 480 & 0.808 & -0.257 & 0.115 & 0.117 & -0.002 & Good \\
\hline 747 & 1-heptanol & 4.239 & 0.240 & 1.659 & 520 & 0.808 & -0.257 & 0.106 & 0.109 & -0.004 & Good \\
\hline 748 & 1-heptanol & 4.239 & 0.240 & 1.659 & 560 & 0.808 & -0.257 & 0.096 & 0.101 & -0.005 & Good \\
\hline 749 & 1-heptanol & 4.239 & 0.240 & 1.659 & 570 & 0.808 & -0.257 & 0.094 & 0.099 & -0.006 & Good \\
\hline
\end{tabular}




\begin{tabular}{|c|c|c|c|c|c|c|c|c|c|c|c|}
\hline 750 & isoheptanol & 4.963 & 0.181 & 2.500 & 240 & 0.828 & -0.020 & 0.147 & 0.142 & 0.005 & Good \\
\hline 751 & isoheptanol & 4.963 & 0.181 & 2.500 & 260 & 0.828 & -0.020 & 0.144 & 0.138 & 0.006 & Good \\
\hline 752 & isoheptanol & 4.963 & 0.181 & 2.500 & 280 & 0.828 & -0.020 & 0.142 & 0.134 & 0.008 & Good \\
\hline 753 & isoheptanol & 4.963 & 0.181 & 2.500 & 360 & 0.828 & -0.020 & 0.130 & 0.118 & 0.012 & Good \\
\hline 754 & isoheptanol & 4.963 & 0.181 & 2.500 & 370 & 0.828 & -0.020 & 0.128 & 0.116 & 0.012 & Good \\
\hline 755 & isoheptanol & 4.963 & 0.181 & 2.500 & 380 & 0.828 & -0.020 & 0.127 & 0.114 & 0.013 & Good \\
\hline 756 & isoheptanol & 4.963 & 0.181 & 2.500 & 400 & 0.828 & -0.020 & 0.124 & 0.110 & 0.014 & Good \\
\hline 757 & isoheptanol & 4.963 & 0.181 & 2.500 & 420 & 0.828 & -0.020 & 0.120 & 0.106 & 0.014 & Good \\
\hline 758 & tert-heptanol & 4.290 & 0.240 & 1.862 & 310 & 0.871 & 0.025 & 0.139 & 0.127 & 0.012 & Good \\
\hline 759 & tert-heptanol & 4.290 & 0.240 & 1.862 & 330 & 0.871 & 0.025 & 0.133 & 0.123 & 0.010 & Good \\
\hline 760 & n-decyl alcohol & 4.408 & 0.211 & 1.522 & 298 & 0.902 & -0.255 & 0.162 & 0.163 & -0.001 & Good \\
\hline 761 & n-decyl alcohol & 4.408 & 0.211 & 1.522 & 373 & 0.902 & -0.255 & 0.151 & 0.148 & 0.003 & Good \\
\hline 762 & n-undecyl alcohol & 4.458 & 0.204 & 1.487 & 340 & 0.927 & -0.255 & 0.163 & 0.157 & 0.006 & Good \\
\hline 763 & n-undecyl alcohol & 4.458 & 0.204 & 1.487 & 380 & 0.927 & -0.255 & 0.157 & 0.149 & 0.008 & Good \\
\hline 764 & n-undecyl alcohol & 4.458 & 0.204 & 1.487 & 440 & 0.927 & -0.255 & 0.148 & 0.137 & 0.011 & Good \\
\hline 765 & n-dodecyl alcohol & 4.506 & 0.198 & 1.457 & 370 & 0.951 & -0.255 & 0.159 & 0.154 & 0.005 & Good \\
\hline 766 & n-dodecyl alcohol & 4.506 & 0.198 & 1.457 & 410 & 0.951 & -0.255 & 0.152 & 0.146 & 0.006 & Good \\
\hline 767 & 1-tetradecanol & 4.596 & 0.189 & 1.405 & 380 & 0.993 & -0.254 & 0.160 & 0.158 & 0.002 & Good \\
\hline 768 & 1-tetradecanol & 4.596 & 0.189 & 1.405 & 410 & 0.993 & -0.254 & 0.154 & 0.152 & 0.002 & Good \\
\hline 769 & 1-tetradecanol & 4.596 & 0.189 & 1.405 & 420 & 0.993 & -0.254 & 0.153 & 0.150 & 0.003 & Good \\
\hline 770 & n-octadecyl alcohol & 4.754 & 0.174 & 1.329 & 350 & 1.062 & -0.253 & 0.173 & 0.174 & -0.001 & Good \\
\hline 771 & n-octadecyl alcohol & 4.754 & 0.174 & 1.329 & 370 & 1.062 & -0.253 & 0.169 & 0.170 & -0.001 & Good \\
\hline
\end{tabular}




\begin{tabular}{|c|c|c|c|c|c|c|c|c|c|c|c|}
\hline 772 & n-octadecyl alcohol & 4.754 & 0.174 & 1.329 & 380 & 1.062 & -0.253 & 0.167 & 0.168 & -0.001 & Good \\
\hline 773 & n-octadecyl alcohol & 4.754 & 0.174 & 1.329 & 410 & 1.062 & -0.253 & 0.161 & 0.162 & -0.001 & Good \\
\hline 774 & n-octadecyl alcohol & 4.754 & 0.174 & 1.329 & 430 & 1.062 & -0.253 & 0.158 & 0.158 & 0.000 & Good \\
\hline 775 & n-octyl alcohol & 4.299 & 0.228 & 1.607 & 300 & 0.843 & -0.256 & 0.157 & 0.156 & 0.001 & Good \\
\hline 776 & n-octyl alcohol & 4.299 & 0.228 & 1.607 & 340 & 0.843 & -0.256 & 0.149 & 0.148 & 0.001 & Good \\
\hline 777 & n-octyl alcohol & 4.299 & 0.228 & 1.607 & 360 & 0.843 & -0.256 & 0.145 & 0.144 & 0.001 & Good \\
\hline 778 & n-octyl alcohol & 4.299 & 0.228 & 1.607 & 380 & 0.843 & -0.256 & 0.141 & 0.140 & 0.001 & Good \\
\hline 779 & n-octyl alcohol & 4.299 & 0.228 & 1.607 & 420 & 0.843 & -0.256 & 0.133 & 0.132 & 0.001 & Good \\
\hline 780 & n-octyl alcohol & 4.299 & 0.228 & 1.607 & 460 & 0.843 & -0.256 & 0.124 & 0.124 & 0.000 & Good \\
\hline 781 & n-octyl alcohol & 4.299 & 0.228 & 1.607 & 540 & 0.843 & -0.256 & 0.105 & 0.108 & -0.003 & Good \\
\hline 782 & n-nonyl alcohol & 4.355 & 0.219 & 1.561 & 300 & 0.874 & -0.256 & 0.159 & 0.159 & 0.000 & Good \\
\hline 783 & n-nonyl alcohol & 4.355 & 0.219 & 1.561 & 340 & 0.874 & -0.256 & 0.151 & 0.151 & 0.000 & Good \\
\hline 784 & n-nonyl alcohol & 4.355 & 0.219 & 1.561 & 420 & 0.874 & -0.256 & 0.135 & 0.135 & 0.000 & Good \\
\hline 785 & n-nonyl alcohol & 4.355 & 0.219 & 1.561 & 500 & 0.874 & -0.256 & 0.116 & 0.119 & -0.003 & Good \\
\hline 786 & diethyl ether & 3.544 & 0.297 & 1.426 & 270 & 0.888 & -0.153 & 0.141 & 0.131 & 0.010 & Good \\
\hline 787 & diethyl ether & 3.544 & 0.297 & 1.426 & 280 & 0.888 & -0.153 & 0.138 & 0.129 & 0.009 & Good \\
\hline 788 & diethyl ether & 3.544 & 0.297 & 1.426 & 290 & 0.888 & -0.153 & 0.134 & 0.127 & 0.007 & Good \\
\hline 789 & dipropyl ether & 3.763 & 0.254 & 1.568 & 320 & 1.030 & -0.184 & 0.125 & 0.117 & 0.008 & Good \\
\hline 790 & dipropyl ether & 3.763 & 0.254 & 1.568 & 340 & 1.030 & -0.184 & 0.118 & 0.113 & 0.005 & Good \\
\hline 791 & ethyl-n-butyl ether & 3.763 & 0.254 & 1.568 & 310 & 1.019 & -0.184 & 0.126 & 0.119 & 0.007 & Good \\
\hline 792 & diamyl ether & 4.096 & 0.211 & 1.480 & 433 & 1.248 & -0.209 & 0.104 & 0.109 & -0.005 & Good \\
\hline 793 & diamyl ether & 4.096 & 0.211 & 1.480 & 453 & 1.248 & -0.209 & 0.101 & 0.105 & -0.004 & Good \\
\hline
\end{tabular}




\begin{tabular}{|c|c|c|c|c|c|c|c|c|c|c|c|}
\hline 794 & ethyl-octyl ether & 4.096 & 0.211 & 1.480 & 280 & 1.179 & -0.209 & 0.134 & 0.140 & -0.006 & Good \\
\hline 795 & di-n-hexyl ether & 4.228 & 0.198 & 1.434 & 280 & 1.331 & -0.216 & 0.138 & 0.146 & -0.008 & Good \\
\hline 796 & di-n-hexyl ether & 4.228 & 0.198 & 1.434 & 300 & 1.331 & -0.216 & 0.134 & 0.142 & -0.008 & Good \\
\hline 797 & di-n-hexyl ether & 4.228 & 0.198 & 1.434 & 320 & 1.331 & -0.216 & 0.131 & 0.138 & -0.007 & Good \\
\hline 798 & di-n-hexyl ether & 4.228 & 0.198 & 1.434 & 340 & 1.331 & -0.216 & 0.127 & 0.134 & -0.007 & Good \\
\hline 799 & di-n-hexyl ether & 4.228 & 0.198 & 1.434 & 360 & 1.331 & -0.216 & 0.123 & 0.130 & -0.007 & Good \\
\hline 800 & di-n-hexyl ether & 4.228 & 0.198 & 1.434 & 380 & 1.331 & -0.216 & 0.120 & 0.126 & -0.006 & Good \\
\hline 801 & di-n-hexyl ether & 4.228 & 0.198 & 1.434 & 400 & 1.331 & -0.216 & 0.116 & 0.122 & -0.006 & Good \\
\hline 802 & di-n-hexyl ether & 4.228 & 0.198 & 1.434 & 420 & 1.331 & -0.216 & 0.113 & 0.118 & -0.005 & Good \\
\hline 803 & di-n-hexyl ether & 4.228 & 0.198 & 1.434 & 450 & 1.331 & -0.216 & 0.107 & 0.112 & -0.005 & Good \\
\hline 804 & di-n-heptyl ether & 4.345 & 0.189 & 1.393 & 290 & 1.404 & -0.221 & 0.139 & 0.152 & -0.013 & Good \\
\hline 805 & di-n-heptyl ether & 4.345 & 0.189 & 1.393 & 320 & 1.404 & -0.221 & 0.134 & 0.146 & -0.012 & Good \\
\hline 806 & di-n-heptyl ether & 4.345 & 0.189 & 1.393 & 340 & 1.404 & -0.221 & 0.131 & 0.142 & -0.011 & Good \\
\hline 807 & di-n-heptyl ether & 4.345 & 0.189 & 1.393 & 500 & 1.404 & -0.221 & 0.102 & 0.110 & -0.008 & Good \\
\hline 808 & octyl ether & 4.450 & 0.181 & 1.359 & 320 & 1.469 & -0.224 & 0.138 & 0.152 & -0.014 & Good \\
\hline 809 & octyl ether & 4.450 & 0.181 & 1.359 & 340 & 1.469 & -0.224 & 0.134 & 0.148 & -0.014 & Good \\
\hline 810 & octyl ether & 4.450 & 0.181 & 1.359 & 400 & 1.469 & -0.224 & 0.124 & 0.136 & -0.012 & Good \\
\hline 811 & octyl ether & 4.450 & 0.181 & 1.359 & 420 & 1.469 & -0.224 & 0.120 & 0.132 & -0.012 & Good \\
\hline 812 & octyl ether & 4.450 & 0.181 & 1.359 & 500 & 1.469 & -0.224 & 0.106 & 0.116 & -0.010 & Good \\
\hline 813 & octyl ether & 4.450 & 0.181 & 1.359 & 520 & 1.469 & -0.224 & 0.103 & 0.112 & -0.009 & Good \\
\hline 814 & dibutyl ether & 3.943 & 0.228 & 1.530 & 300 & 1.149 & -0.200 & 0.131 & 0.128 & 0.004 & Good \\
\hline 815 & Ethyl hexyl ether & 3.931 & 0.242 & 1.619 & 320 & 0.945 & -0.217 & 0.121 & 0.126 & -0.005 & Good \\
\hline
\end{tabular}




\begin{tabular}{|c|c|c|c|c|c|c|c|c|c|c|c|}
\hline 816 & Ethyl hexyl ether & 3.931 & 0.242 & 1.619 & 360 & 0.945 & -0.217 & 0.112 & 0.118 & -0.006 & Good \\
\hline 817 & Isobutyric aldehyde & 4.320 & 0.335 & 2.035 & 360 & 1.573 & -0.034 & 0.136 & 0.133 & 0.003 & Good \\
\hline 818 & Isobutyric aldehyde & 4.320 & 0.335 & 2.035 & 400 & 1.573 & -0.034 & 0.131 & 0.125 & 0.006 & Good \\
\hline 819 & valeraldehyde & 4.354 & 0.300 & 2.092 & 300 & 1.571 & -0.219 & 0.139 & 0.145 & -0.006 & Good \\
\hline 820 & valeraldehyde & 4.354 & 0.300 & 2.092 & 340 & 1.571 & -0.219 & 0.129 & 0.137 & -0.008 & Good \\
\hline 821 & valeraldehyde & 4.354 & 0.300 & 2.092 & 375 & 1.571 & -0.219 & 0.119 & 0.130 & -0.011 & Good \\
\hline 822 & valeraldehyde & 4.354 & 0.300 & 2.092 & 380 & 1.571 & -0.219 & 0.119 & 0.129 & -0.010 & Good \\
\hline 823 & decyl aldehyde & 4.595 & 0.221 & 1.717 & 280 & 1.971 & -0.241 & 0.148 & 0.158 & -0.010 & Good \\
\hline 824 & decyl aldehyde & 4.595 & 0.221 & 1.717 & 300 & 1.971 & -0.241 & 0.142 & 0.154 & -0.012 & Good \\
\hline 825 & decyl aldehyde & 4.595 & 0.221 & 1.717 & 330 & 1.971 & -0.241 & 0.138 & 0.148 & -0.010 & Good \\
\hline 826 & decyl aldehyde & 4.595 & 0.221 & 1.717 & 360 & 1.971 & -0.241 & 0.132 & 0.142 & -0.010 & Good \\
\hline 827 & decyl aldehyde & 4.595 & 0.221 & 1.717 & 390 & 1.971 & -0.241 & 0.126 & 0.136 & -0.010 & Good \\
\hline 828 & lauric aldehyde & 4.678 & 0.206 & 1.626 & 350 & 2.080 & -0.243 & 0.142 & 0.149 & -0.007 & Good \\
\hline 829 & lauric aldehyde & 4.678 & 0.206 & 1.626 & 410 & 2.080 & -0.243 & 0.131 & 0.137 & -0.006 & Good \\
\hline 830 & Acetaldehyde & 4.174 & 0.491 & 2.128 & 298 & 1.131 & -0.094 & 0.181 & 0.186 & -0.005 & Good \\
\hline 831 & propionaldehyde & 4.238 & 0.390 & 2.161 & 323 & 1.304 & -0.174 & 0.153 & 0.156 & -0.003 & Good \\
\hline 832 & heptanal & 4.457 & 0.256 & 1.909 & 273 & 1.762 & -0.233 & 0.144 & 0.153 & -0.009 & Good \\
\hline 833 & heptanal & 4.457 & 0.256 & 1.909 & 298 & 1.762 & -0.233 & 0.138 & 0.148 & -0.010 & Good \\
\hline 834 & octanal & 4.505 & 0.242 & 1.835 & 273 & 1.839 & -0.236 & 0.144 & 0.155 & -0.011 & Good \\
\hline 835 & octanal & 4.505 & 0.242 & 1.835 & 288 & 1.839 & -0.236 & 0.141 & 0.152 & -0.011 & Good \\
\hline 836 & nonanal & 4.551 & 0.230 & 1.772 & 298 & 1.908 & -0.239 & 0.140 & 0.152 & -0.012 & Good \\
\hline 837 & 2-methyl hexaldehyde & 4.477 & 0.256 & 1.966 & 273 & 1.942 & -0.133 & 0.143 & 0.143 & 0.000 & Good \\
\hline
\end{tabular}




\begin{tabular}{|c|c|c|c|c|c|c|c|c|c|c|c|}
\hline 838 & 3-methyl hexaldehyde & 4.477 & 0.256 & 2.091 & 273 & 1.876 & -0.133 & 0.148 & 0.137 & 0.011 & Good \\
\hline 839 & Formic acid & 4.575 & 0.655 & 2.322 & 330 & 1.357 & -0.470 & 0.264 & 0.266 & -0.002 & Moderate \\
\hline 840 & Acetic acid & 4.627 & 0.500 & 2.406 & 310 & 1.803 & 0.358 & 0.162 & 0.169 & -0.007 & Good \\
\hline 841 & Acetic acid & 4.627 & 0.500 & 2.406 & 320 & 1.803 & 0.358 & 0.160 & 0.167 & -0.007 & Good \\
\hline 842 & Propionic acid & 4.668 & 0.415 & 2.404 & 290 & 2.021 & 0.154 & 0.145 & 0.160 & -0.015 & Good \\
\hline 843 & Propionic acid & 4.668 & 0.415 & 2.404 & 310 & 2.021 & 0.154 & 0.143 & 0.156 & -0.013 & Good \\
\hline 844 & Propionic acid & 4.668 & 0.415 & 2.404 & 320 & 2.021 & 0.154 & 0.142 & 0.154 & -0.012 & Good \\
\hline 845 & Propionic acid & 4.668 & 0.415 & 2.404 & 360 & 2.021 & 0.154 & 0.138 & 0.146 & -0.008 & Good \\
\hline 846 & Propionic acid & 4.668 & 0.415 & 2.404 & 370 & 2.021 & 0.154 & 0.136 & 0.144 & -0.008 & Good \\
\hline 847 & Propionic acid & 4.668 & 0.415 & 2.404 & 390 & 2.021 & 0.154 & 0.134 & 0.140 & -0.006 & Good \\
\hline 848 & valeric acid & 4.745 & 0.326 & 2.292 & 360 & 2.353 & -0.002 & 0.132 & 0.136 & -0.004 & Good \\
\hline 849 & valeric acid & 4.745 & 0.326 & 2.292 & 390 & 2.353 & -0.002 & 0.128 & 0.130 & -0.002 & Good \\
\hline 850 & valeric acid & 4.745 & 0.326 & 2.292 & 440 & 2.353 & -0.002 & 0.122 & 0.120 & 0.002 & Good \\
\hline 851 & valeric acid & 4.745 & 0.326 & 2.292 & 450 & 2.353 & -0.002 & 0.120 & 0.118 & 0.002 & Good \\
\hline 852 & caproic acid & 4.781 & 0.300 & 2.181 & 280 & 2.481 & -0.041 & 0.145 & 0.153 & -0.008 & Good \\
\hline 853 & caproic acid & 4.781 & 0.300 & 2.181 & 310 & 2.481 & -0.041 & 0.139 & 0.147 & -0.008 & Good \\
\hline 854 & caproic acid & 4.781 & 0.300 & 2.181 & 320 & 2.481 & -0.041 & 0.137 & 0.145 & -0.008 & Good \\
\hline 855 & caproic acid & 4.781 & 0.300 & 2.181 & 330 & 2.481 & -0.041 & 0.134 & 0.143 & -0.009 & Good \\
\hline 856 & enanthic acid & 4.816 & 0.280 & 2.084 & 300 & 2.592 & -0.069 & 0.140 & 0.151 & -0.011 & Good \\
\hline 857 & enanthic acid & 4.816 & 0.280 & 2.084 & 340 & 2.592 & -0.069 & 0.135 & 0.143 & -0.008 & Good \\
\hline 858 & capric acid & 4.914 & 0.240 & 1.864 & 450 & 2.856 & -0.120 & 0.127 & 0.127 & 0.000 & Good \\
\hline 859 & Undecanoic acid & 4.945 & 0.230 & 1.808 & 310 & 2.928 & -0.131 & 0.155 & 0.156 & -0.001 & Good \\
\hline
\end{tabular}




\begin{tabular}{|c|c|c|c|c|c|c|c|c|c|c|c|}
\hline 860 & Undecanoic acid & 4.945 & 0.230 & 1.808 & 320 & 2.928 & -0.131 & 0.154 & 0.154 & 0.000 & Good \\
\hline 861 & lauric acid & 4.975 & 0.222 & 1.759 & 320 & 2.994 & -0.141 & 0.162 & 0.157 & 0.005 & Good \\
\hline 862 & myristic acid & 5.032 & 0.209 & 1.677 & 360 & 3.112 & -0.155 & 0.154 & 0.152 & 0.002 & Good \\
\hline 863 & myristic acid & 5.032 & 0.209 & 1.677 & 380 & 3.112 & -0.155 & 0.150 & 0.148 & 0.002 & Good \\
\hline 864 & myristic acid & 5.032 & 0.209 & 1.677 & 420 & 3.112 & -0.155 & 0.142 & 0.140 & 0.002 & Good \\
\hline 865 & myristic acid & 5.032 & 0.209 & 1.677 & 440 & 3.112 & -0.155 & 0.138 & 0.136 & 0.002 & Good \\
\hline 866 & myristic acid & 5.032 & 0.209 & 1.677 & 450 & 3.112 & -0.155 & 0.136 & 0.134 & 0.002 & Good \\
\hline 867 & palmitic acid & 5.086 & 0.199 & 1.610 & 380 & 3.215 & -0.167 & 0.157 & 0.152 & 0.005 & Good \\
\hline 868 & palmitic acid & 5.086 & 0.199 & 1.610 & 390 & 3.215 & -0.167 & 0.155 & 0.150 & 0.005 & Good \\
\hline 869 & palmitic acid & 5.086 & 0.199 & 1.610 & 470 & 3.215 & -0.167 & 0.138 & 0.134 & 0.004 & Good \\
\hline 870 & stearic acid & 5.137 & 0.191 & 1.555 & 410 & 3.306 & -0.176 & 0.157 & 0.149 & 0.008 & Good \\
\hline 871 & stearic acid & 5.137 & 0.191 & 1.555 & 430 & 3.306 & -0.176 & 0.152 & 0.145 & 0.007 & Good \\
\hline 872 & caprylic acid & 4.850 & 0.264 & 2.000 & 320 & 2.690 & -0.090 & 0.143 & 0.149 & -0.006 & Good \\
\hline 873 & caprylic acid & 4.850 & 0.264 & 2.000 & 360 & 2.690 & -0.090 & 0.137 & 0.141 & -0.004 & Good \\
\hline 874 & nonanoic acid & 4.882 & 0.251 & 1.927 & 320 & 2.777 & -0.107 & 0.146 & 0.151 & -0.005 & Good \\
\hline 875 & nonanoic acid & 4.882 & 0.251 & 1.927 & 340 & 2.777 & -0.107 & 0.143 & 0.147 & -0.004 & Good \\
\hline 876 & nonanoic acid & 4.882 & 0.251 & 1.927 & 360 & 2.777 & -0.107 & 0.140 & 0.143 & -0.003 & Good \\
\hline 877 & nonanoic acid & 4.882 & 0.251 & 1.927 & 380 & 2.777 & -0.107 & 0.137 & 0.139 & -0.002 & Good \\
\hline 878 & 2-pentanone & 4.366 & 0.300 & 1.924 & 280 & 1.693 & 0.022 & 0.147 & 0.143 & 0.004 & Good \\
\hline 879 & 2-pentanone & 4.366 & 0.300 & 1.924 & 340 & 1.693 & 0.022 & 0.130 & 0.131 & -0.001 & Good \\
\hline 880 & diethyl ketone & 4.366 & 0.300 & 1.674 & 280 & 1.718 & 0.022 & 0.149 & 0.155 & -0.006 & Good \\
\hline 881 & diethyl ketone & 4.366 & 0.300 & 1.674 & 290 & 1.718 & 0.022 & 0.146 & 0.153 & -0.007 & Good \\
\hline
\end{tabular}




\begin{tabular}{|c|c|c|c|c|c|c|c|c|c|c|c|}
\hline 882 & diethyl ketone & 4.366 & 0.300 & 1.674 & 300 & 1.718 & 0.022 & 0.143 & 0.151 & -0.008 & Good \\
\hline 883 & diethyl ketone & 4.366 & 0.300 & 1.674 & 320 & 1.718 & 0.022 & 0.137 & 0.147 & -0.010 & Good \\
\hline 884 & diethyl ketone & 4.366 & 0.300 & 1.674 & 360 & 1.718 & 0.022 & 0.126 & 0.139 & -0.013 & Good \\
\hline 885 & 2-hexanone & 4.418 & 0.275 & 1.878 & 280 & 1.800 & -0.026 & 0.153 & 0.143 & 0.010 & Good \\
\hline 886 & 2-hexanone & 4.418 & 0.275 & 1.878 & 340 & 1.800 & -0.026 & 0.127 & 0.131 & -0.004 & Good \\
\hline 887 & 2-hexanone & 4.418 & 0.275 & 1.878 & 440 & 1.800 & -0.026 & 0.095 & 0.111 & -0.016 & Good \\
\hline 888 & 4-heptanone & 4.468 & 0.256 & 1.764 & 300 & 1.975 & -0.060 & 0.136 & 0.142 & -0.006 & Good \\
\hline 889 & 4-heptanone & 4.468 & 0.256 & 1.764 & 320 & 1.975 & -0.060 & 0.131 & 0.138 & -0.007 & Good \\
\hline 890 & 4-heptanone & 4.468 & 0.256 & 1.764 & 380 & 1.975 & -0.060 & 0.119 & 0.126 & -0.007 & Good \\
\hline 891 & 4-heptanone & 4.468 & 0.256 & 1.764 & 400 & 1.975 & -0.060 & 0.115 & 0.122 & -0.007 & Good \\
\hline 892 & 3-heptanone & 4.468 & 0.256 & 1.764 & 390 & 1.953 & -0.060 & 0.114 & 0.125 & -0.011 & Good \\
\hline 893 & diamyl ketone & 4.646 & 0.213 & 1.624 & 290 & 2.152 & -0.131 & 0.139 & 0.153 & -0.014 & Good \\
\hline 894 & diamyl ketone & 4.646 & 0.213 & 1.624 & 300 & 2.152 & -0.131 & 0.137 & 0.151 & -0.014 & Good \\
\hline 895 & diamyl ketone & 4.646 & 0.213 & 1.624 & 320 & 2.152 & -0.131 & 0.133 & 0.147 & -0.014 & Good \\
\hline 896 & diamyl ketone & 4.646 & 0.213 & 1.624 & 340 & 2.152 & -0.131 & 0.129 & 0.143 & -0.014 & Good \\
\hline 897 & diamyl ketone & 4.646 & 0.213 & 1.624 & 350 & 2.152 & -0.131 & 0.127 & 0.141 & -0.014 & Good \\
\hline 898 & di-n-hexyl ketone & 4.725 & 0.200 & 1.567 & 330 & 2.523 & -0.149 & 0.136 & 0.146 & -0.010 & Good \\
\hline 899 & di-n-hexyl ketone & 4.725 & 0.200 & 1.567 & 340 & 2.523 & -0.149 & 0.134 & 0.144 & -0.010 & Good \\
\hline 900 & di-n-hexyl ketone & 4.725 & 0.200 & 1.567 & 390 & 2.523 & -0.149 & 0.124 & 0.134 & -0.010 & Good \\
\hline 901 & di-n-hexyl ketone & 4.725 & 0.200 & 1.567 & 400 & 2.523 & -0.149 & 0.122 & 0.132 & -0.010 & Good \\
\hline 902 & di-n-hexyl ketone & 4.725 & 0.200 & 1.567 & 440 & 2.523 & -0.149 & 0.114 & 0.124 & -0.010 & Good \\
\hline 903 & di-n-hexyl ketone & 4.725 & 0.200 & 1.567 & 470 & 2.523 & -0.149 & 0.108 & 0.118 & -0.010 & Good \\
\hline
\end{tabular}




\begin{tabular}{|c|c|c|c|c|c|c|c|c|c|c|c|}
\hline 904 & 2-octanone & 4.516 & 0.242 & 1.764 & 280 & 1.967 & -0.084 & 0.139 & 0.147 & -0.008 & Good \\
\hline 905 & 2-octanone & 4.516 & 0.242 & 1.764 & 340 & 1.967 & -0.084 & 0.125 & 0.135 & -0.010 & Good \\
\hline 906 & 2-octanone & 4.516 & 0.242 & 1.764 & 370 & 1.967 & -0.084 & 0.119 & 0.129 & -0.010 & Good \\
\hline 907 & 2-octanone & 4.516 & 0.242 & 1.764 & 420 & 1.967 & -0.084 & 0.107 & 0.119 & -0.012 & Good \\
\hline 908 & acetone & 4.251 & 0.390 & 1.571 & 300 & 1.412 & 0.232 & 0.160 & 0.167 & -0.007 & Good \\
\hline 909 & Methyl isopropyl ketone & 4.387 & 0.300 & 1.799 & 233 & 1.799 & 0.159 & 0.159 & 0.151 & 0.008 & Good \\
\hline 910 & Methyl isopropyl ketone & 4.387 & 0.300 & 1.799 & 253 & 1.799 & 0.159 & 0.153 & 0.147 & 0.006 & Good \\
\hline 911 & Methyl isopropyl ketone & 4.387 & 0.300 & 1.799 & 293 & 1.799 & 0.159 & 0.142 & 0.139 & 0.003 & Good \\
\hline 912 & Methyl isobutyl ketone & 4.461 & 0.275 & 1.733 & 253 & 2.058 & 0.319 & 0.148 & 0.135 & 0.013 & Good \\
\hline 913 & Methyl isobutyl ketone & 4.461 & 0.275 & 1.733 & 273 & 2.058 & 0.319 & 0.143 & 0.131 & 0.012 & Good \\
\hline 914 & Methyl isobutyl ketone & 4.461 & 0.275 & 1.733 & 333 & 2.058 & 0.319 & 0.127 & 0.119 & 0.008 & Good \\
\hline 915 & Ethyl formate & 4.425 & 0.415 & 2.404 & 300 & 2.044 & -0.106 & 0.159 & 0.156 & 0.003 & Good \\
\hline 916 & ethyl acetate & 4.485 & 0.362 & 2.093 & 280 & 2.428 & 0.170 & 0.149 & 0.143 & 0.006 & Good \\
\hline 917 & ethyl acetate & 4.485 & 0.362 & 2.093 & 320 & 2.428 & 0.170 & 0.137 & 0.135 & 0.002 & Good \\
\hline 918 & ethyl acetate & 4.485 & 0.362 & 2.093 & 330 & 2.428 & 0.170 & 0.134 & 0.133 & 0.001 & Good \\
\hline 919 & ethyl propionate & 4.532 & 0.326 & 2.016 & 350 & 2.640 & 0.088 & 0.120 & 0.128 & -0.008 & Good \\
\hline 920 & propyl acetate & 4.532 & 0.326 & 2.133 & 280 & 2.596 & 0.088 & 0.144 & 0.136 & 0.008 & Good \\
\hline 921 & propyl acetate & 4.532 & 0.326 & 2.133 & 320 & 2.596 & 0.088 & 0.135 & 0.128 & 0.007 & Good \\
\hline 922 & propyl acetate & 4.532 & 0.326 & 2.133 & 330 & 2.596 & 0.088 & 0.133 & 0.126 & 0.007 & Good \\
\hline 923 & propyl acetate & 4.532 & 0.326 & 2.133 & 350 & 2.596 & 0.088 & 0.129 & 0.122 & 0.007 & Good \\
\hline 924 & methyl valerate & 4.577 & 0.300 & 2.071 & 320 & 2.872 & -0.001 & 0.133 & 0.129 & 0.004 & Good \\
\hline 925 & methyl valerate & 4.577 & 0.300 & 2.071 & 350 & 2.872 & -0.001 & 0.126 & 0.123 & 0.003 & Good \\
\hline
\end{tabular}




\begin{tabular}{|c|c|c|c|c|c|c|c|c|c|c|c|}
\hline 926 & butyl acetate & 4.577 & 0.300 & 2.071 & 270 & 2.738 & 0.035 & 0.143 & 0.138 & 0.005 & Good \\
\hline 927 & butyl acetate & 4.577 & 0.300 & 2.071 & 280 & 2.738 & 0.035 & 0.140 & 0.136 & 0.004 & Good \\
\hline 928 & butyl acetate & 4.577 & 0.300 & 2.071 & 290 & 2.738 & 0.035 & 0.138 & 0.134 & 0.004 & Good \\
\hline 929 & isobutyl acetate & 4.594 & 0.300 & 2.071 & 290 & 2.831 & 0.134 & 0.123 & 0.128 & -0.005 & Good \\
\hline 930 & isobutyl acetate & 4.594 & 0.300 & 2.071 & 300 & 2.831 & 0.134 & 0.119 & 0.126 & -0.007 & Good \\
\hline 931 & isobutyl acetate & 4.594 & 0.300 & 2.071 & 320 & 2.831 & 0.134 & 0.112 & 0.122 & -0.010 & Good \\
\hline 932 & amyl formate & 4.567 & 0.300 & 2.181 & 320 & 2.512 & -0.181 & 0.130 & 0.137 & -0.007 & Good \\
\hline 933 & amyl formate & 4.567 & 0.300 & 2.181 & 340 & 2.512 & -0.181 & 0.127 & 0.133 & -0.006 & Good \\
\hline 934 & amyl formate & 4.567 & 0.300 & 2.181 & 380 & 2.512 & -0.181 & 0.120 & 0.125 & -0.005 & Good \\
\hline 935 & ethyl butyrate & 4.577 & 0.300 & 2.071 & 350 & 2.816 & 0.035 & 0.125 & 0.121 & 0.004 & Good \\
\hline 936 & n-propyl propionate & 4.577 & 0.300 & 2.071 & 330 & 2.808 & 0.035 & 0.130 & 0.125 & 0.005 & Good \\
\hline 937 & n-propyl propionate & 4.577 & 0.300 & 2.071 & 340 & 2.808 & 0.035 & 0.126 & 0.123 & 0.003 & Good \\
\hline 938 & n-amyl acetate & 4.620 & 0.280 & 1.999 & 300 & 2.858 & -0.004 & 0.133 & 0.134 & -0.001 & Good \\
\hline 939 & n-butyl propionate & 4.620 & 0.280 & 2.032 & 300 & 2.949 & -0.004 & 0.139 & 0.131 & 0.008 & Good \\
\hline 940 & n-hexyl formate & 4.610 & 0.280 & 2.084 & 340 & 2.626 & -0.191 & 0.128 & 0.135 & -0.007 & Good \\
\hline 941 & n-hexyl butyrate & 4.738 & 0.240 & 1.873 & 300 & 3.351 & -0.074 & 0.137 & 0.135 & 0.002 & Good \\
\hline 942 & n-hexyl butyrate & 4.738 & 0.240 & 1.873 & 340 & 3.351 & -0.074 & 0.129 & 0.127 & 0.002 & Good \\
\hline 943 & n-hexyl butyrate & 4.738 & 0.240 & 1.873 & 420 & 3.351 & -0.074 & 0.111 & 0.111 & 0.000 & Good \\
\hline 944 & n-hexyl butyrate & 4.738 & 0.240 & 1.873 & 480 & 3.351 & -0.074 & 0.099 & 0.099 & 0.000 & Good \\
\hline 945 & n-heptyl propionate & 4.738 & 0.240 & 1.873 & 300 & 3.268 & -0.074 & 0.137 & 0.136 & 0.001 & Good \\
\hline 946 & n-heptyl propionate & 4.738 & 0.240 & 1.873 & 360 & 3.268 & -0.074 & 0.124 & 0.124 & 0.000 & Good \\
\hline 947 & n-octyl acetate & 4.738 & 0.240 & 1.814 & 330 & 3.139 & -0.074 & 0.134 & 0.134 & 0.000 & Good \\
\hline
\end{tabular}




\begin{tabular}{|c|c|c|c|c|c|c|c|c|c|c|c|}
\hline 948 & n-octyl acetate & 4.738 & 0.240 & 1.814 & 350 & 3.139 & -0.074 & 0.126 & 0.130 & -0.004 & Good \\
\hline 949 & n-octyl propionate & 4.775 & 0.230 & 1.826 & 360 & 3.350 & -0.089 & 0.122 & 0.126 & -0.004 & Good \\
\hline 950 & n-octyl propionate & 4.775 & 0.230 & 1.826 & 380 & 3.350 & -0.089 & 0.118 & 0.122 & -0.004 & Good \\
\hline 951 & n-octyl propionate & 4.775 & 0.230 & 1.826 & 400 & 3.350 & -0.089 & 0.115 & 0.118 & -0.003 & Good \\
\hline 952 & heptylbutanoate & 4.775 & 0.230 & 1.826 & 500 & 3.444 & -0.089 & 0.096 & 0.096 & 0.000 & Good \\
\hline 953 & n-decyl acetate & 4.810 & 0.222 & 1.721 & 350 & 3.283 & -0.102 & 0.126 & 0.135 & -0.009 & Good \\
\hline 954 & n-octyl butyrate & 4.810 & 0.222 & 1.782 & 320 & 3.527 & -0.102 & 0.135 & 0.135 & 0.000 & Good \\
\hline 955 & n-octyl butyrate & 4.810 & 0.222 & 1.782 & 340 & 3.527 & -0.102 & 0.131 & 0.131 & 0.000 & Good \\
\hline 956 & n-octyl butyrate & 4.810 & 0.222 & 1.782 & 500 & 3.527 & -0.102 & 0.102 & 0.099 & 0.003 & Good \\
\hline 957 & methyl stearate & 5.027 & 0.187 & 1.509 & 320 & 3.882 & -0.167 & 0.152 & 0.153 & -0.001 & Good \\
\hline 958 & methyl stearate & 5.027 & 0.187 & 1.509 & 330 & 3.882 & -0.167 & 0.151 & 0.151 & 0.000 & Good \\
\hline 959 & methyl stearate & 5.027 & 0.187 & 1.509 & 340 & 3.882 & -0.167 & 0.149 & 0.149 & 0.000 & Good \\
\hline 960 & methyl stearate & 5.027 & 0.187 & 1.509 & 360 & 3.882 & -0.167 & 0.146 & 0.145 & 0.001 & Good \\
\hline 961 & methyl stearate & 5.027 & 0.187 & 1.509 & 380 & 3.882 & -0.167 & 0.143 & 0.141 & 0.002 & Good \\
\hline 962 & methyl stearate & 5.027 & 0.187 & 1.509 & 390 & 3.882 & -0.167 & 0.142 & 0.139 & 0.003 & Good \\
\hline 963 & methyl stearate & 5.027 & 0.187 & 1.509 & 400 & 3.882 & -0.167 & 0.140 & 0.137 & 0.003 & Good \\
\hline 964 & methyl stearate & 5.027 & 0.187 & 1.509 & 430 & 3.882 & -0.167 & 0.136 & 0.131 & 0.005 & Good \\
\hline 965 & n-octadecyl acetate & 5.054 & 0.184 & 1.488 & 350 & 3.686 & -0.159 & 0.164 & 0.151 & 0.013 & Good \\
\hline 966 & n-octadecyl acetate & 5.054 & 0.184 & 1.488 & 360 & 3.686 & -0.159 & 0.161 & 0.149 & 0.012 & Good \\
\hline 967 & n-octadecyl acetate & 5.054 & 0.184 & 1.488 & 370 & 3.686 & -0.159 & 0.158 & 0.147 & 0.011 & Good \\
\hline 968 & n-octadecyl acetate & 5.054 & 0.184 & 1.488 & 390 & 3.686 & -0.159 & 0.153 & 0.143 & 0.010 & Good \\
\hline 969 & n-butyl stearate & 5.107 & 0.178 & 1.504 & 320 & 4.180 & -0.167 & 0.154 & 0.152 & 0.002 & Good \\
\hline
\end{tabular}




\begin{tabular}{|c|c|c|c|c|c|c|c|c|c|c|c|}
\hline 970 & n-butyl stearate & 5.107 & 0.178 & 1.504 & 330 & 4.180 & -0.167 & 0.152 & 0.150 & 0.002 & Good \\
\hline 971 & n-butyl stearate & 5.107 & 0.178 & 1.504 & 440 & 4.180 & -0.167 & 0.135 & 0.128 & 0.007 & Good \\
\hline 972 & n-amyl propionate & 4.700 & 0.251 & 1.925 & 404 & 3.246 & -0.056 & 0.116 & 0.112 & 0.004 & Good \\
\hline 973 & n-hexyl acetate & 4.661 & 0.264 & 1.931 & 380 & 2.963 & -0.033 & 0.116 & 0.120 & -0.004 & Good \\
\hline 974 & n-heptyl formate & 4.652 & 0.264 & 2.000 & 310 & 2.727 & -0.199 & 0.135 & 0.142 & -0.007 & Good \\
\hline 975 & n-heptyl formate & 4.652 & 0.264 & 2.000 & 370 & 2.727 & -0.199 & 0.124 & 0.130 & -0.006 & Good \\
\hline 976 & isoamyl propionate & 4.677 & 0.264 & 2.056 & 300 & 3.138 & 0.045 & 0.129 & 0.124 & 0.005 & Good \\
\hline 977 & isoamyl propionate & 4.677 & 0.264 & 2.056 & 320 & 3.138 & 0.045 & 0.125 & 0.120 & 0.005 & Good \\
\hline 978 & isoamyl propionate & 4.677 & 0.264 & 2.056 & 340 & 3.138 & 0.045 & 0.122 & 0.116 & 0.006 & Good \\
\hline 979 & isoamyl propionate & 4.677 & 0.264 & 2.056 & 400 & 3.138 & 0.045 & 0.111 & 0.104 & 0.007 & Good \\
\hline 980 & isoamyl propionate & 4.677 & 0.264 & 2.056 & 440 & 3.138 & 0.045 & 0.104 & 0.096 & 0.008 & Good \\
\hline 981 & Ethyl hexanoate & 4.661 & 0.264 & 1.979 & 280 & 3.087 & -0.033 & 0.141 & 0.136 & 0.005 & Good \\
\hline 982 & Ethyl hexanoate & 4.661 & 0.264 & 1.979 & 360 & 3.087 & -0.033 & 0.126 & 0.120 & 0.006 & Good \\
\hline 983 & n-amylbutyrate & 4.700 & 0.251 & 1.925 & 280 & 3.246 & -0.056 & 0.143 & 0.137 & 0.006 & Good \\
\hline 984 & n-amylbutyrate & 4.700 & 0.251 & 1.925 & 300 & 3.246 & -0.056 & 0.139 & 0.133 & 0.006 & Good \\
\hline 985 & n-amylbutyrate & 4.700 & 0.251 & 1.925 & 360 & 3.246 & -0.056 & 0.128 & 0.121 & 0.007 & Good \\
\hline 986 & n-amylbutyrate & 4.700 & 0.251 & 1.925 & 380 & 3.246 & -0.056 & 0.124 & 0.117 & 0.007 & Good \\
\hline 987 & n-hexyl propionate & 4.700 & 0.251 & 1.925 & 300 & 3.175 & -0.056 & 0.136 & 0.134 & 0.002 & Good \\
\hline 988 & n-hexyl propionate & 4.700 & 0.251 & 1.925 & 320 & 3.175 & -0.056 & 0.133 & 0.130 & 0.003 & Good \\
\hline 989 & n-hexyl propionate & 4.700 & 0.251 & 1.925 & 340 & 3.175 & -0.056 & 0.130 & 0.126 & 0.004 & Good \\
\hline 990 & n-octyl formate & 4.691 & 0.251 & 1.927 & 340 & 2.818 & -0.205 & 0.126 & 0.138 & -0.012 & Good \\
\hline 991 & n-heptylacetate & 4.700 & 0.251 & 1.869 & 340 & 3.056 & -0.056 & 0.124 & 0.130 & -0.006 & Good \\
\hline
\end{tabular}




\begin{tabular}{|c|c|c|c|c|c|c|c|c|c|c|c|c|}
\hline & 992 & n-heptylacetate & 4.700 & 0.251 & 1.869 & 380 & 3.056 & -0.056 & 0.115 & 0.122 & -0.007 & Good \\
\hline \multirow{21}{*}{$\begin{array}{c}\text { Prediction } \\
\text { set }\end{array}$} & & & & & & & & & & & & / \\
\hline & 993 & Undecylic aldehyde* & 4.637 & 0.213 & 1.669 & 290 & 2.027 & -0.242 & 0.152 & 0.159 & -0.007 & \\
\hline & 994 & Undecylic aldehyde* & 4.637 & 0.213 & 1.669 & 300 & 2.027 & -0.242 & 0.149 & 0.157 & -0.007 & I \\
\hline & 995 & Undecylic aldehyde* & 4.637 & 0.213 & 1.669 & 310 & 2.027 & -0.242 & 0.149 & 0.155 & -0.006 & / \\
\hline & 996 & Undecylic aldehyde* & 4.637 & 0.213 & 1.669 & 320 & 2.027 & -0.242 & 0.148 & 0.153 & -0.005 & / \\
\hline & 997 & Undecylic aldehyde* & 4.637 & 0.213 & 1.669 & 330 & 2.027 & -0.242 & 0.147 & 0.151 & -0.004 & / \\
\hline & 998 & Dodecyl aldehyde* & 4.678 & 0.206 & 1.626 & 290 & 2.080 & -0.243 & 0.156 & 0.161 & -0.005 & / \\
\hline & 999 & Dodecyl aldehyde* & 4.678 & 0.206 & 1.626 & 300 & 2.080 & -0.243 & 0.154 & 0.159 & -0.005 & / \\
\hline & 1000 & Dodecyl aldehyde* & 4.678 & 0.206 & 1.626 & 310 & 2.080 & -0.243 & 0.152 & 0.157 & -0.005 & / \\
\hline & 1001 & Dodecyl aldehyde* & 4.678 & 0.206 & 1.626 & 320 & 2.080 & -0.243 & 0.150 & 0.155 & -0.005 & / \\
\hline & 1002 & Dodecyl aldehyde* & 4.678 & 0.206 & 1.626 & 330 & 2.080 & -0.243 & 0.148 & 0.153 & -0.005 & / \\
\hline & 1003 & 2-Heptanone* & 4.468 & 0.256 & 1.820 & 290 & 1.889 & -0.060 & 0.146 & 0.143 & 0.003 & / \\
\hline & 1004 & 2-Heptanone* & 4.468 & 0.256 & 1.820 & 300 & 1.889 & -0.060 & 0.145 & 0.141 & 0.004 & / \\
\hline & 1005 & 2-Heptanone* & 4.468 & 0.256 & 1.820 & 310 & 1.889 & -0.060 & 0.144 & 0.139 & 0.005 & / \\
\hline & 1006 & 2-Heptanone* & 4.468 & 0.256 & 1.820 & 320 & 1.889 & -0.060 & 0.143 & 0.137 & 0.007 & / \\
\hline & 1007 & 2-Heptanone* & 4.468 & 0.256 & 1.820 & 330 & 1.889 & -0.060 & 0.142 & 0.135 & 0.007 & / \\
\hline & 1008 & 2-Nonanone* & 4.561 & 0.230 & 1.712 & 290 & 2.036 & -0.103 & 0.145 & 0.148 & -0.003 & / \\
\hline & 1009 & 2-Nonanone* & 4.561 & 0.230 & 1.712 & 300 & 2.036 & -0.103 & 0.144 & 0.146 & -0.002 & / \\
\hline & 1010 & 2-Nonanone* & 4.561 & 0.230 & 1.712 & 310 & 2.036 & -0.103 & 0.143 & 0.144 & 0.000 & / \\
\hline & 1011 & 2-Nonanone* & 4.561 & 0.230 & 1.712 & 320 & 2.036 & -0.103 & 0.143 & 0.142 & 0.001 & / \\
\hline & 1012 & 2-Nonanone* & 4.561 & 0.230 & 1.712 & 330 & 2.036 & -0.103 & 0.142 & 0.140 & 0.002 & / \\
\hline
\end{tabular}




\begin{tabular}{|c|c|c|c|c|c|c|c|c|c|c|c|}
\hline 1013 & 2-Undecanone* & 4.646 & 0.213 & 1.624 & 290 & 2.152 & -0.131 & 0.149 & 0.153 & -0.004 & / \\
\hline 1014 & 2-Undecanone* & 4.646 & 0.213 & 1.624 & 300 & 2.152 & -0.131 & 0.146 & 0.151 & -0.005 & / \\
\hline 1015 & 2-Undecanone* & 4.646 & 0.213 & 1.624 & 310 & 2.152 & -0.131 & 0.143 & 0.149 & -0.006 & / \\
\hline 1016 & 2-Undecanone* & 4.646 & 0.213 & 1.624 & 320 & 2.152 & -0.131 & 0.142 & 0.147 & -0.005 & / \\
\hline 1017 & 2-Undecanone* & 4.646 & 0.213 & 1.624 & 330 & 2.152 & -0.131 & 0.140 & 0.145 & -0.005 & / \\
\hline 1018 & n-Butyric acid* & 4.707 & 0.362 & 2.404 & 280 & 2.203 & 0.056 & 0.148 & 0.152 & -0.004 & / \\
\hline 1019 & n-Butyric acid* & 4.707 & 0.362 & 2.404 & 290 & 2.203 & 0.056 & 0.147 & 0.150 & -0.003 & / \\
\hline 1020 & n-Butyric acid* & 4.707 & 0.362 & 2.404 & 300 & 2.203 & 0.056 & 0.146 & 0.148 & -0.002 & / \\
\hline 1021 & n-Butyric acid* & 4.707 & 0.362 & 2.404 & 310 & 2.203 & 0.056 & 0.144 & 0.146 & -0.002 & / \\
\hline 1022 & n-Butyric acid* & 4.707 & 0.362 & 2.404 & 320 & 2.203 & 0.056 & 0.142 & 0.144 & -0.002 & / \\
\hline 1023 & n-Butyric acid* & 4.707 & 0.362 & 2.404 & 330 & 2.203 & 0.056 & 0.140 & 0.142 & -0.002 & / \\
\hline & 3,5,5-Trimethylhexanoic & & & & & & & & & & / \\
\hline 1024 & $\operatorname{acid}^{*}$ & 4.922 & 0.251 & 2.186 & 310 & 3.077 & 0.173 & 0.118 & 0.122 & -0.004 & \\
\hline & 3,5,5-Trimethylhexanoic & & & & & & & & & & / \\
\hline 1025 & $\operatorname{acid}^{*}$ & 4.922 & 0.251 & 2.186 & 320 & 3.077 & 0.173 & 0.119 & 0.120 & -0.001 & \\
\hline & 3,5,5-Trimethylhexanoic & & & & & & & & & & / \\
\hline 1026 & $\operatorname{acid}^{*}$ & 4.922 & 0.251 & 2.186 & 330 & 3.077 & 0.173 & 0.120 & 0.118 & 0.002 & \\
\hline 1027 & 2-Methylbutyric Acid* & 4.759 & 0.326 & 2.292 & 290 & 2.531 & 0.114 & 0.139 & 0.142 & -0.003 & / \\
\hline 1028 & 2-Methylbutyric Acid* & 4.759 & 0.326 & 2.292 & 310 & 2.531 & 0.114 & 0.136 & 0.138 & -0.002 & / \\
\hline 1029 & 2-Methylbutyric Acid* & 4.759 & 0.326 & 2.292 & 330 & 2.531 & 0.114 & 0.132 & 0.134 & -0.002 & / \\
\hline 1030 & 2,6-dimethyl heptanol* & 4.397 & 0.219 & 1.833 & 320 & 0.976 & -0.106 & 0.140 & 0.134 & 0.006 & / \\
\hline 1031 & 2,6-dimethyl heptanol* & 4.397 & 0.219 & 1.833 & 340 & 0.976 & -0.106 & 0.135 & 0.130 & 0.005 & / \\
\hline
\end{tabular}




\begin{tabular}{|c|c|c|c|c|c|c|c|c|c|c|c|}
\hline 1032 & 2,6-dimethyl heptanol* & 4.397 & 0.219 & 1.833 & 360 & 0.976 & -0.106 & 0.133 & 0.126 & 0.007 & 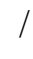 \\
\hline 1033 & 2,6-dimethyl heptanol* & 4.397 & 0.219 & 1.833 & 380 & 0.976 & -0.106 & 0.130 & 0.122 & 0.008 & / \\
\hline 1034 & 2,6-dimethyl heptanol* & 4.397 & 0.219 & 1.833 & 400 & 0.976 & -0.106 & 0.128 & 0.118 & 0.010 & / \\
\hline 1035 & 3,5,5-trimethyl-1-hexanol* & 4.421 & 0.219 & 1.883 & 410 & 0.981 & 0.044 & 0.118 & 0.107 & 0.011 & 1 \\
\hline 1036 & 3,5,5-trimethyl-1-hexanol* & 4.421 & 0.219 & 1.883 & 420 & 0.981 & 0.044 & 0.115 & 0.105 & 0.010 & r \\
\hline 1037 & 3,5,5-trimethyl-1-hexanol* & 4.421 & 0.219 & 1.883 & 430 & 0.981 & 0.044 & 0.114 & 0.103 & 0.011 & t \\
\hline 1038 & 3,5,5-trimethyl-1-hexanol* & 4.421 & 0.219 & 1.883 & 280 & 0.981 & 0.044 & 0.140 & 0.133 & 0.007 & \\
\hline 1039 & 3,5,5-trimethyl-1-hexanol* & 4.421 & 0.219 & 1.883 & 300 & 0.981 & 0.044 & 0.137 & 0.129 & 0.008 & \\
\hline 1040 & 4-Methyl-2-pentanol* & 4.227 & 0.254 & 1.875 & 290 & 0.850 & -0.003 & 0.136 & 0.132 & 0.004 & \\
\hline 1041 & 4-Methyl-2-pentanol* & 4.227 & 0.254 & 1.875 & 300 & 0.850 & -0.003 & 0.133 & 0.130 & 0.003 & \\
\hline 1042 & 4-Methyl-2-pentanol* & 4.227 & 0.254 & 1.875 & 310 & 0.850 & -0.003 & 0.132 & 0.128 & 0.004 & \\
\hline 1043 & 4-Methyl-2-pentanol* & 4.227 & 0.254 & 1.875 & 320 & 0.850 & -0.003 & 0.130 & 0.126 & 0.004 & 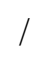 \\
\hline 1044 & 4-Methyl-2-pentanol* & 4.227 & 0.254 & 1.875 & 330 & 0.850 & -0.003 & 0.129 & 0.124 & 0.005 & 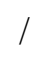 \\
\hline 1045 & Isoamyl acetate* & 4.636 & 0.280 & 2.119 & 290 & 2.926 & 0.083 & 0.122 & 0.125 & -0.002 & 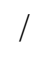 \\
\hline 1046 & Isoamyl acetate* & 4.636 & 0.280 & 2.119 & 300 & 2.926 & 0.083 & 0.120 & 0.123 & -0.003 & \\
\hline 1047 & Isoamyl acetate* & 4.636 & 0.280 & 2.119 & 310 & 2.926 & 0.083 & 0.117 & 0.121 & -0.003 & \\
\hline 1048 & Isoamyl acetate* & 4.636 & 0.280 & 2.119 & 320 & 2.926 & 0.083 & 0.115 & 0.119 & -0.004 & \\
\hline 1049 & Isoamyl acetate* & 4.636 & 0.280 & 2.119 & 330 & 2.926 & 0.083 & 0.112 & 0.117 & -0.004 & \\
\hline 1050 & isobutyl formate* & 4.540 & 0.326 & 2.292 & 290 & 2.482 & -0.049 & 0.151 & 0.136 & 0.015 & \\
\hline 1051 & isobutyl formate* & 4.540 & 0.326 & 2.292 & 300 & 2.482 & -0.049 & 0.148 & 0.134 & 0.014 & \\
\hline 1052 & isobutyl formate* & 4.540 & 0.326 & 2.292 & 310 & 2.482 & -0.049 & 0.145 & 0.132 & 0.013 & / \\
\hline 1053 & isobutyl formate* & 4.540 & 0.326 & 2.292 & 320 & 2.482 & -0.049 & 0.142 & 0.130 & 0.012 & 1 \\
\hline
\end{tabular}




\begin{tabular}{|c|c|c|c|c|c|c|c|c|c|c|c|}
\hline 1054 & isobutyl formate* & 4.540 & 0.326 & 2.292 & 330 & 2.482 & -0.049 & 0.139 & 0.128 & 0.011 & I \\
\hline 1055 & Ethyl tert-amyl ether* & 3.932 & 0.240 & 1.585 & 290 & 1.138 & 0.157 & 0.118 & 0.109 & 0.010 & I \\
\hline 1056 & Ethyl tert-amyl ether* & 3.932 & 0.240 & 1.585 & 300 & 1.138 & 0.157 & 0.116 & 0.107 & 0.009 & / \\
\hline 1057 & Ethyl tert-amyl ether* & 3.932 & 0.240 & 1.585 & 310 & 1.138 & 0.157 & 0.113 & 0.105 & 0.009 & 1 \\
\hline 1058 & Ethyl tert-amyl ether* & 3.932 & 0.240 & 1.585 & 320 & 1.138 & 0.157 & 0.111 & 0.103 & 0.008 & 1 \\
\hline 1059 & Ethyl tert-amyl ether* & 3.932 & 0.240 & 1.585 & 330 & 1.138 & 0.157 & 0.108 & 0.101 & 0.008 & 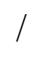 \\
\hline 1060 & Methyl isopropyl ether* & 3.591 & 0.297 & 1.559 & 290 & 0.993 & -0.001 & 0.122 & 0.114 & 0.008 & 1 \\
\hline 1061 & Methyl isopropyl ether* & 3.591 & 0.297 & 1.559 & 300 & 0.993 & -0.001 & 0.118 & 0.112 & 0.007 & / \\
\hline 1062 & Methyl isopropyl ether* & 3.591 & 0.297 & 1.559 & 310 & 0.993 & -0.001 & 0.115 & 0.110 & 0.005 & / \\
\hline 1063 & Methyl isopropyl ether* & 3.591 & 0.297 & 1.559 & 320 & 0.993 & -0.001 & 0.111 & 0.108 & 0.003 & / \\
\hline 1064 & Methyl isopropyl ether* & 3.591 & 0.297 & 1.559 & 330 & 0.993 & -0.001 & 0.107 & 0.106 & 0.001 & I \\
\hline
\end{tabular}

\section{References:}

(1) Vargaftik, N. B., Handbook of Thermal Conductivity of Liquids and Gases, CRC Press: Boca Raton FL, 1994; 358. 